\title{
Measurement of the associated production of a $Z$ boson with charm or bottom quark jets in proton-proton collisions at $\sqrt{s}=13 \mathrm{TeV}$
}

\author{
A. M. Sirunyan et al. \\ (CMS Collaboration)
}

(Received 19 January 2020; accepted 13 July 2020; published 19 August 2020)

\begin{abstract}
Ratios of cross sections, $\sigma(Z+c$ jets $) / \sigma(Z+$ jets $), \sigma(Z+b$ jets $) / \sigma(Z+$ jets $)$, and $\sigma(Z+c$ jets $) /$ $\sigma(Z+b$ jets $)$ in the associated production of a $Z$ boson with at least one charm or bottom quark jet are measured in proton-proton collisions at $\sqrt{s}=13 \mathrm{TeV}$. The data sample, collected by the CMS experiment at the CERN LHC, corresponds to an integrated luminosity of $35.9 \mathrm{fb}^{-1}$, with a fiducial volume of $p_{\mathrm{T}}>30 \mathrm{GeV}$ and $|\eta|<2.4$ for the jets, where $p_{\mathrm{T}}$ and $\eta$ represent transverse momentum and pseudorapidity, respectively. The $Z$ boson candidates come from leptonic decays into electrons or muons with $p_{\mathrm{T}}>25 \mathrm{GeV}$ and $|\eta|<2.4$, and the dilepton mass satisfies $71<m_{Z}<111 \mathrm{GeV}$. The measured values are $\sigma(Z+c$ jets $) / \sigma(Z+$ jets $)=0.102 \pm 0.002 \pm 0.009, \sigma(Z+b$ jets $) / \sigma(Z+$ jets $)=$ $0.0633 \pm 0.0004 \pm 0.0015$, and $\sigma(Z+c$ jets $) / \sigma(Z+b$ jets $)=1.62 \pm 0.03 \pm 0.15$. Results on the inclusive and differential cross section ratios as functions of jet and $Z$ boson transverse momentum are compared with predictions from leading and next-to-leading order perturbative quantum chromodynamics calculations. These are the first measurements of the cross section ratios at $13 \mathrm{TeV}$.
\end{abstract}

DOI: 10.1103/PhysRevD.102.032007

\section{INTRODUCTION}

Studies of $Z$ boson production in association with heavy-flavor (HF) jets from the hadronization of heavy $(c$ or $b$ ) quarks provide important tests of perturbative quantum chromodynamics (pQCD) calculations. A good description of these processes is also important since they form a major background for a variety of physics processes including Higgs boson production in association with a $Z$ boson, $Z H(H \rightarrow c \bar{c}$ or $H \rightarrow b \bar{b})$, and searches for new physics signatures in final states with leptons and HF jets. Two different approaches are currently available for calculating the $Z+\mathrm{HF}$ jets production: the five-flavor scheme (5FS) [1] and the four-flavor scheme (4FS) [2]. Both approaches yield consistent results within theoretical uncertainties [3].

Several $Z+$ HF jets measurements have been performed by the CDF and D0 Collaborations at the FNAL Tevatron [4-6] and by the ATLAS, CMS, and LHCb Collaborations at the CERN LHC [7-10]. The D0 Collaboration reported on the first $\sigma(Z+c$ jets $) /$ $\sigma(Z+b$ jets $)$ cross section ratio measurement [5] and

*Full author list given at the end of the article.

Published by the American Physical Society under the terms of the Creative Commons Attribution 4.0 International license. Further distribution of this work must maintain attribution to the author(s) and the published article's title, journal citation, and DOI. Funded by SCOAP. observed a significantly higher value compared to next-toleading order (NLO) pQCD calculations. A measurement of the $\sigma(Z+c$ jets $) / \sigma(Z+b$ jets $)$ cross section ratio in $8 \mathrm{TeV}$ proton-proton $(p p)$ collisions at the LHC has been recently reported by the CMS Collaboration [11] and is in agreement with predictions from leading order (LO) and NLO calculations obtained with the MadGraph [12] and MadGraph5_aMC@NLO [13] programs, respectively.

The current paper reports on simultaneous measurements of the $c$ and $b$ quark jet contents in a sample containing a $Z$ boson (in the following, $Z$ is used as a shorthand for $Z / \gamma^{*}$ ) produced in association with at least one jet. These measurements provide the first results for proton-proton collisions at $\sqrt{s}=13 \mathrm{TeV}$. The experimental precision is improved with respect to previous LHC results because of the increased size of the data sample and advanced heavyflavor tagging techniques. The $Z$ bosons are identified through reconstructed dielectrons or dimuons, where the individual leptons are subject to requirements on transverse momentum $\left(p_{\mathrm{T}}>25 \mathrm{GeV}\right)$ and pseudorapidity $(|\eta|<2.4)$. The dilepton invariant mass must be within a $Z$ boson window of 71-111 GeV, and jets are required to have $p_{\mathrm{T}}>$ $30 \mathrm{GeV}$ and $|\eta|<2.4$.

The following cross section ratios are measured: $\sigma(Z+c$ jets $) / \sigma(Z+$ jets $), \sigma(Z+b$ jets $) / \sigma(Z+$ jets $)$, and $\sigma(Z+c$ jets $) / \sigma(Z+b$ jets $)$. These cross section ratios are measured inclusively and differentially as functions of the transverse momentum of the jet and the $Z$ boson, and are unfolded to the particle level taking into account detector effects. The measurements of the cross section ratios 
benefit from cancellations of several systematic uncertainties related to the jet, lepton, and luminosity measurements. A number of theory-related uncertainties are reduced as well, including those linked to the details of parton showering and hadronization. Therefore, by measuring cross section ratios one can more precisely compare data with theoretical calculations.

The paper is organized as follows. The CMS experiment and data together with simulated samples used in the analysis are described in Secs. II and III. Details of the measurements are described in Secs. IV, V, and VI, while Secs. VII and VIII present the systematic uncertainties and the measurement results, respectively, followed by a summary in Sec. IX.

\section{THE CMS DETECTOR}

The central feature of the CMS apparatus is a superconducting solenoid of $6 \mathrm{~m}$ internal diameter, providing a magnetic field of $3.8 \mathrm{~T}$. Within the solenoid volume are a silicon pixel and strip tracker, covering a pseudorapidity region of $|\eta|<2.5$, a lead tungstate crystal electromagnetic calorimeter (ECAL), and a brass and scintillator hadron calorimeter, each composed of a barrel and two endcap sections. Forward calorimeters, made of steel and quartz fibers, extend the pseudorapidity coverage provided by the barrel and endcap detectors to $|\eta|<5$. Muons are detected in gas-ionization chambers embedded in the steel fluxreturn yoke outside the solenoid and covering $|\eta|<2.4$.

Events of interest are selected using a two-tiered trigger system [14]. The first level, composed of custom hardware processors, uses information from the calorimeters and muon detectors to select events at a rate of around $100 \mathrm{kHz}$ within a time interval of less than $4 \mu \mathrm{s}$. The second level, known as the high-level trigger, consists of a farm of processors running a version of the full event reconstruction software optimized for fast processing, and reduces the event rate to around $1 \mathrm{kHz}$ before data storage.

A more detailed description of the CMS detector, together with a definition of the coordinate system used and the relevant kinematic variables, can be found in Ref. [15].

\section{DATA AND SIMULATED SAMPLES}

The cross section ratio measurements are based on proton-proton $(p p)$ collision data at $\sqrt{s}=13 \mathrm{TeV}$ collected with the CMS detector in 2016 and corresponding to an integrated luminosity of $35.9 \mathrm{fb}^{-1}$ [16]. Recorded events have an average 23 additional $p p$ interactions per bunch crossing (pileup) together with the hard process.

Various Monte Carlo (MC) event generators are used to simulate the $Z+$ jets signal and background processes. The full detector simulation is based on the GEANT4 package [17]. The simulation includes the pileup effects from the same or nearby bunch crossings by overlapping the hard process of interest with the pileup events. The simulated events are reconstructed with the same algorithms as used for the data.

The $Z+$ jets events are generated by MadGraph5_aMC@NLO v2.2.2 [13] (using 5FS; denoted as MG5_aMC in the following) at NLO in PQCD with up to two additional partons at the matrix element level, generated for each parton multiplicity and then merged. The MG5_aMC matrix element generator is interfaced with PYTHIA v8.212 [18], which simulates the parton shower and hadronization processes, through the FxFx merging scheme [19] at a matching scale of $19 \mathrm{GeV}$. The predicted numbers of events from $Z+$ jets production are estimated using a cross section at next-to-next-to-leading order (NNLO) accuracy obtained from FEWZ v3.1 [20].

The background events originate from top quark and diboson processes. Top quark-antiquark $(t \bar{t})$ production, which forms the dominant background, is generated at NLO by POWHEG v2.0 [21-24] and normalized to a cross section calculated by using TOP++ v2.0 [25] at NNLO accuracy including soft-gluon resummation. The diboson $(W W, W Z, Z Z)$ backgrounds are generated by PYTHIA while POWHEG and NLO MG5_aMC are used to simulate the single top quark processes ( $s$-channel, $t$-channel, and $t W$ ). The POWHEG generator is also interfaced with PYTHIA for parton showering and hadronization. The diboson and single top quark predictions are normalized to NNLO $[26,27]$ cross sections.

The NNPDF 3.0 NLO and LO parton distribution functions (PDF) [28] are used for the NLO and LO calculations, respectively. PYTHIA uses the NNPDF 2.3 LO PDF set and the CUETP8M1 [29] or CUETP8M2T4 [30] ( $t \bar{t}$ sample) for the underlying event tune.

\section{OBJECT RECONSTRUCTION AND EVENT SELECTION}

The particle-flow (PF) algorithm [31] reconstructs and identifies each individual particle in an event, with an optimized combination of information from the various elements of the CMS detector. The neutral particle energy deposits are determined in the calorimeters, whereas charged tracks are measured in the central tracking and muon systems.

The candidate vertex with the largest value of summed physics-object $p_{\mathrm{T}}^{2}$ is taken to be the primary $p p$ interaction vertex. The physics objects are the jets, clustered using the jet finding algorithm [32,33] with the tracks assigned to candidate vertices as inputs, and leptons. More details are given in Ref. [34].

Electrons are reconstructed using momentum measurements in the tracker combined with the energy deposits in the ECAL [35]. The identification requirements are based on the ECAL shower shape, matching between the electron track and the energy clusters in the ECAL, and observables characterizing the bremsstrahlung along the electron trajectory. Electrons are required to originate from the primary 
vertex. The electron momentum is estimated by combining the energy measurement in the ECAL with the momentum measurement in the tracker. The momentum resolution for electrons with $p_{\mathrm{T}} \approx 45 \mathrm{GeV}$ from $Z \rightarrow e^{+} e^{-}$decays ranges from 1.7 to $4.5 \%$. The resolution tends to be better in the barrel region than in the endcaps, and it also depends on the bremsstrahlung energy emitted by the electron as it traverses the material in front of the ECAL. The dielectron mass resolution for $Z \rightarrow e^{+} e^{-}$decays when both electrons are in the ECAL barrel is $1.9 \%$, and is $2.9 \%$ when both electrons are in the endcaps [35].

Muon candidates are built by combining signals from the tracker and the muon subsystems. The identification criteria are based on the number of measurements in the detectors, the fit quality of the track, and requirements on its association with the primary vertex. Matching muons to tracks measured in the tracker results in a relative transverse momentum resolution, for muons with $p_{\mathrm{T}}$ up to $100 \mathrm{GeV}$, of $1 \%$ in the barrel and 3\% in the endcaps [36].

To reduce the misidentification rate, electrons and muons are required to be isolated. Activity near an electron (muon) is quantified as the sum of transverse momenta of $\mathrm{PF}$ candidates within the isolation cone radius of $\Delta R=$ $\sqrt{(\Delta \eta)^{2}+(\Delta \phi)^{2}}=0.3(0.4)$ around the electron (muon) track, where $\phi$ is the azimuthal angle. After compensating for the energy contribution from pileup in the isolation cone, the resultant sum is required to be less than $25 \%$ of the lepton transverse momentum. The lepton isolation, along with other requirements to select $Z+$ jets events, strongly suppresses background events with misidentified dileptons such as $W+$ jets and QCD multijets.

Based on the PF candidates, jets are reconstructed using the anti- $k_{\mathrm{T}}$ algorithm with a distance parameter of 0.4 . Jet momentum is determined as the vector sum of all particle momenta in the jet; based on simulation this is, on average, within 5 to $10 \%$ of the true jet momentum over the entire $p_{\mathrm{T}}$ spectrum and detector acceptance. Pileup interactions can result in more tracks and calorimetric energy depositions, increasing the apparent jet momentum. To mitigate this effect, tracks originating from pileup vertices are discarded and an offset correction is applied to account for remaining contributions $[37,38]$. Jet energy corrections are derived from simulation studies so that the average measured response of jets becomes identical to that of particle-level jets. In situ measurements of the momentum balance in dijet, photon + jet, $Z+$ jet, and multijet events are used to determine any residual differences between the jet energy scale (JES) in data and in simulation, and appropriate corrections are applied [39]. The jet energy resolution (JER) typically amounts to $16 \%$ at $30 \mathrm{GeV}$ and $8 \%$ at $100 \mathrm{GeV}$. Additional selection criteria are applied to remove jets potentially dominated by instrumental effects or reconstruction failures [40].

For this analysis, the selection for $Z+$ jets events starts with the trigger requirements based on two electron (muon) objects identified by the trigger system that pass $p_{\mathrm{T}}$ thresholds of 23 and $13 \mathrm{GeV}(17$ and $8 \mathrm{GeV})$. The $Z+$ jets events are further selected by requiring two reconstructed electrons or muons with $p_{\mathrm{T}}>25 \mathrm{GeV}$ and within $|\eta|<2.4$. The $p_{\mathrm{T}}$ requirement is chosen to obtain high trigger efficiency for selecting the signal events. Events containing two selected electrons (muons) are categorized in the electron (muon) channel. The lepton candidates are subject to requirements on their transverse impact parameter, $\left|d_{x y}\right|<0.05 \mathrm{~cm}$, and their longitudinal impact parameter, $\left|d_{z}\right|<0.2 \mathrm{~cm}$, both with respect to the primary vertex. The $Z$ boson candidate is reconstructed from a pair of oppositely charged same-flavor leptons with invariant mass between 71 and $111 \mathrm{GeV}$. An event must contain at least one associated jet with $p_{\mathrm{T}}>30 \mathrm{GeV}$ and $|\eta|<2.4$.

Missing transverse momentum is used in this analysis to reduce background contributions from $t \bar{t}$ and single top quark production processes. In contrast to the $Z+$ jets, these processes have a significant amount of missing energy because of undetected neutrinos in top quark decays. The missing transverse momentum vector, $\vec{p}_{\mathrm{T}}^{\text {miss }}$, is computed as the negative vector sum of the transverse momenta of all PF candidates in an event [41] and is further modified to account for corrections to the energy scale of the reconstructed jets. Its magnitude, $p_{\mathrm{T}}^{\text {miss }}$, is required to be less than $40 \mathrm{GeV}$, which results in a signal efficiency of $\approx 80 \%$ with the $t \bar{t}$ rejection factor of $\approx 4.8$.

A $Z+$ jets sample with enriched $c$ and $b$ quark jet content is selected by applying an HF tagging requirement to jets in the $Z+$ jets sample described above. The discrimination of HF jets from light-flavor quark and gluon jets, referred to as light jets in the following, is achieved by constructing a discriminator variable from tracks and secondary vertex (SV) characteristics. Artificial neural network algorithms are used to combine specific properties of the HF quarks, long lifetime and substantial mass, to build the discriminator. The algorithm used in the analysis, the combined secondary vertex (Version 2), is described in Ref. [42]. Some of the important input variables are the number of secondary vertices and the number of tracks associated with each of them, the mass and 2D decay distance significance of the SV with the smallest decay distance uncertainty, and the signed 3D impact parameter significance of the tracks. Here the significance is defined as the ratio between a measured quantity and its uncertainty. Although the combined secondary vertex (Version 2) is trained to distinguish $b$ jets, it does occasionally tag a $c$ jet. Therefore, at a proper operating point, the algorithm can retain a sufficient amount of $c$ jets while heavily suppressing light jets. The analysis uses a "medium" operating point, which corresponds to approximately 10 (60)\% tagging efficiencies for $c(b)$ quark jets and a misidentification probability of $1 \%$ for a light jet. The $Z+$ HF jets sample must contain at least one tagged jet. The tagging efficiencies are determined using MC samples and 
TABLE I. Numbers of events that satisfy the $Z+$ jets and $Z+$ HF jets selection criteria in the electron and muon channels. The uncertainties are statistical only.

\begin{tabular}{|c|c|c|c|c|}
\hline & \multicolumn{2}{|c|}{$Z+$ jets sample } & \multicolumn{2}{|c|}{$Z+\mathrm{HF}$ jets sample } \\
\hline & Electron & Muon & Electron & Muon \\
\hline$Z+c$ jets & $171970 \pm 530$ & $287090 \pm 720$ & $18870 \pm 170$ & $32310 \pm 230$ \\
\hline$Z+b$ jets & $95910 \pm 410$ & $159500 \pm 560$ & $60100 \pm 310$ & $100630 \pm 420$ \\
\hline$Z+$ light jets & $1531900 \pm 1600$ & $2612100 \pm 2200$ & $6170 \pm 100$ & $10810 \pm 140$ \\
\hline$t \bar{t}$ & $5850 \pm 50$ & $9440 \pm 60$ & $3850 \pm 40$ & $6180 \pm 50$ \\
\hline Diboson & $10040 \pm 60$ & $16310 \pm 80$ & $780 \pm 20$ & $1320 \pm 20$ \\
\hline Single $t$ & $580 \pm 10$ & $950 \pm 10$ & $303 \pm 7$ & $500 \pm 10$ \\
\hline Total, simulation & $1816200 \pm 1700$ & $3085400 \pm 2400$ & $90070 \pm 370$ & $151740 \pm 510$ \\
\hline Data & 1759047 & 2959629 & 79015 & 130775 \\
\hline Data/simulation & $0.969 \pm 0.001$ & $0.959 \pm 0.001$ & $0.877 \pm 0.005$ & $0.862 \pm 0.004$ \\
\hline
\end{tabular}

corrected for the difference between data and simulation. The corresponding correction factors are derived from the data versus simulation efficiency comparisons in dedicated control samples containing $t \bar{t}$ and multijet events [42].

In simulation, the classification of reconstructed $Z+$ jets events into $Z+c$ jets, $Z+b$ jets, and $Z+$ light jets categories is based on the flavors of reconstructed jets with $p_{\mathrm{T}}>30 \mathrm{GeV}$ and $|\eta|<2.4$. They are classified as $c$ or $b$ jets if they are matched to MC generated $c$ or $b$ hadrons [42]. In the case where both $c$ and $b$ hadrons are matched, the jet is considered a $b$ jet. Based on reconstructed jets with defined flavors, events are classified as $Z+b$ jets if they contain at least one $b$ jet. Of the remaining events, those that contain at least one $c$ hadron are considered as $Z+c$ jets and those that contain neither $c$ nor $b$ hadrons are classified as $Z+$ light jets.

Table I lists the number of events estimated in simulation and found in data that satisfy the $Z+$ jets and $Z+$ HF jets selection criteria for both the electron and muon channels. The background, mostly from top quark and diboson processes, is approximately $5 \%$ in the $Z+$ HF jets sample, and the diboson background is dominated by the $W Z$ events.

\section{CROSS SECTION RATIO MEASUREMENTS}

\section{A. Analysis strategy}

The goal of the analysis is to precisely measure the fraction of jets with heavy flavors in $Z+$ jets events. For this purpose, the SV invariant mass, $M_{\mathrm{SV}}$, of the tagged jet with highest $p_{\mathrm{T}}$ in the $Z+\mathrm{HF}$ jets events is used. The SV is reconstructed using an adaptive vertex reconstruction algorithm [43] from selected tracks within a cone of $\Delta R<$ 0.3 around the jet axis. The distance between the track and the jet axis measured at their point of closest approach must be less than $0.2 \mathrm{~cm}$. Details of track selections and SV reconstructions can be found in Refs. $[42,44]$. The $M_{\mathrm{SV}}$ is calculated using the momenta of charged-particle tracks associated with the SV. The corresponding particles are assumed to have the pion mass for the purpose of calculating the SV mass. The $M_{\mathrm{SV}}$ distributions possess specific features depending on the jet flavor, and can be used as templates in a fit to the $M_{\mathrm{SV}}$ distribution in data to extract the fractions of $c$ and $b$ jets, as discussed in Sec. V C.

The template fit is performed in the $Z+$ jets sample enriched with HF jets, i.e., in the $Z+\mathrm{HF}$ jets sample, and the observed number of $Z+c$ jets $\left(N_{c}\right)$ and $Z+b$ jets $\left(N_{b}\right)$ events are derived. They are corrected for the efficiencies of tagging events, $\epsilon_{\text {tag }}^{c}$ and $\epsilon_{\text {tag }}^{b}$ for $N_{c}$ and $N_{b}$, respectively, to obtain the numbers of $Z+c$ jets and $Z+b$ jets events in the $Z+$ jets sample. The cross section ratios are then calculated as

$$
\begin{aligned}
& \mathrm{R}(c / \mathrm{j})=\frac{\sigma(Z+c \text { jets })}{\sigma(Z+\text { jets })}=\frac{N_{c}}{N_{\text {jet }} \epsilon_{\text {tag }}^{c}}, \\
& \mathrm{R}(b / \mathrm{j})=\frac{\sigma(Z+b \text { jets })}{\sigma(Z+\text { jets })}=\frac{N_{b}}{N_{\text {jet }} \epsilon_{\text {tag }}^{b}}, \\
& \mathrm{R}(c / b)=\frac{\sigma(Z+c \text { jets })}{\sigma(Z+b \text { jets })}=\frac{N_{c} \epsilon_{\mathrm{tag}}^{b}}{N_{b} \epsilon_{\mathrm{tag}}^{c}},
\end{aligned}
$$

where $N_{\text {jet }}$ is the number of selected $Z+$ jets events remaining after subtracting background contributions ( $t \bar{t}$, diboson, and single top) from data. These backgrounds are estimated using simulation. In the above formulas, the integrated luminosity as well as the efficiencies that are related to lepton and $p_{\mathrm{T}}^{\text {miss }}$ requirements in the $Z+$ jets event selection cancel.

For the differential measurements, the same procedure described here is applied in each jet or $Z$ boson $p_{\mathrm{T}}$ interval. Dedicated $M_{\mathrm{SV}}$ templates are derived for each interval to take into account the dependence of the $M_{\mathrm{SV}}$ shape on jet kinematic variables. Finally, the cross section ratios are unfolded for various experimental effects, most notably the detector resolution and efficiencies. 

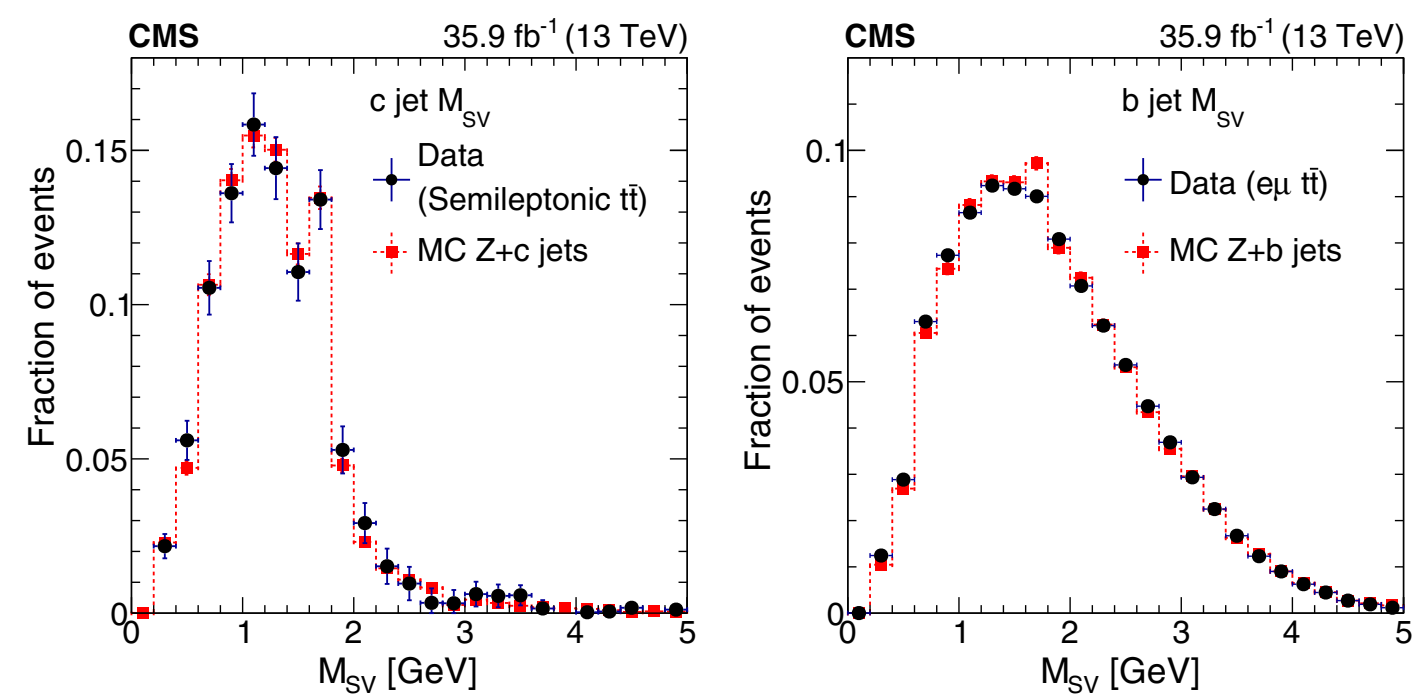

FIG. 1. Comparisons of $c$ jet (left) and $b$ jet (right) $M_{\mathrm{SV}}$ distributions for data and simulation. A shape correction is applied to the datadriven distribution in the right panel to account for the difference between the jets in $t \bar{t}$ and $Z+b$ jets processes.

\section{B. $Z+\mathrm{HF}$ jets event tagging efficiency}

The efficiencies of tagging $Z+\mathrm{HF}$ jets events, $\epsilon_{\mathrm{tag}}^{c}$ and $\epsilon_{\text {tag }}^{b}$, are calculated as the ratio between numbers of selected $Z+c$ jets and $Z+b$ jets events, respectively, in the $Z+$ HF jets and the $Z+$ jets samples. They are estimated using simulations, which are corrected with data. In the jet $p_{\mathrm{T}}$ range between 30 and $200 \mathrm{GeV}$ the efficiencies vary only slightly and range from 8.3 to $11.3 \%$ for $Z+c$ jets and from 45.9 to $60.7 \%$ for $Z+b$ jets events. The $Z+$ light jets mistagging rate increases from 0.3 to $1.0 \%$ in the same $p_{\mathrm{T}}$ range.

\section{Estimation of the event yields}

A binned maximum likelihood template fit, based on $M_{\mathrm{SV}}$ distributions of the leading $p_{\mathrm{T}}$ HF-tagged jets, is used to obtain the numbers of $Z+c$ jets and $Z+b$ jets events in the $Z+\mathrm{HF}$ jets sample. The parameters of interest are the scale factors, $\mathrm{SF}_{c}$ and $\mathrm{SF}_{b}$, that adjust the $\mathrm{MC}$ rates to fit the data, while their uncertainties are treated as nuisance parameters. The $M_{\mathrm{SV}}$ distributions of the simulated $Z+c$ jets, $Z+b$ jets, and $Z+$ light jets categories are normalized to the integrated luminosity of the data sample using an NNLO cross section for the total $Z+$ jets rate. The top quark and diboson backgrounds, which contribute about $5 \%$ of the events in the $Z+$ HF jets sample, are estimated from simulation. The predicted yields of all these processes are shown in Table I.

For each $M_{\mathrm{SV}}$ bin a Poisson distribution is constructed from the number of observed events, with its mean taken from MC predictions of signal $(Z+c$ jets and $Z+b$ jets $)$ and background $(Z+$ light jets, top quark, and diboson) yields. The likelihood is the product of the Poisson distributions and Gaussian (or log-normal) distributions, where the latter are used to constrain the nuisance parameters. The choice of Gaussian or log-normal constraints depends on whether the corresponding systematic uncertainty affects the shape or normalization of the templates, respectively. To obtain a combined result, the electron and muon channel data are fitted simultaneously using a common set of scale factors. After the fit, the numbers of $Z+c$ jets and $Z+b$ jets events, $N_{c}$ and $N_{b}$, are obtained from the MC predictions scaled by the $\mathrm{SF}_{c}$ and $\mathrm{SF}_{b}$ factors.

The $M_{\mathrm{SV}}$ template of $c$ jets in the $Z+c$ jets events is obtained from simulation. The $c$ jet $M_{\mathrm{SV}}$ shape is validated with a $t \bar{t}$-enriched data sample where only one of the $W$ bosons decays to leptons. The other $W$ boson decays hadronically with a branching fraction of $33 \%$ for a charm quark in the final state. The event selection requires a wellidentified and isolated muon having $p_{\mathrm{T}}>25 \mathrm{GeV}$ and $|\eta|<2.4$ together with at least four jets, each with $p_{\mathrm{T}}>$ $30 \mathrm{GeV}$ and $|\eta|<2.4$. The $c$ and $b$ jet identification is performed with the following procedure. To reduce the combinatorics the best pair (triplet) of jets is chosen by minimizing the reconstructed and nominal mass of the $W$ boson (top quark). From this optimization, the $c$ and $b$ jet candidates from top quark and $W$ boson decays are identified. The event is kept if these candidates pass the jet HF tagging requirement described in Sec. IV. In the resulting sample of the $c$ jet candidates about half have correct flavor assignment whereas the other half constitute mostly $b$ jets that are misidentified as $c$ jets. The $c$ jet $M_{\mathrm{SV}}$ distribution in data is found by subtracting the backgrounds containing $b$ jets and light jets in the sample.

The $c$ jet $M_{\mathrm{SV}}$ template from simulation is compared with that observed in the validation sample and agreement is found within the statistical uncertainties as shown in 


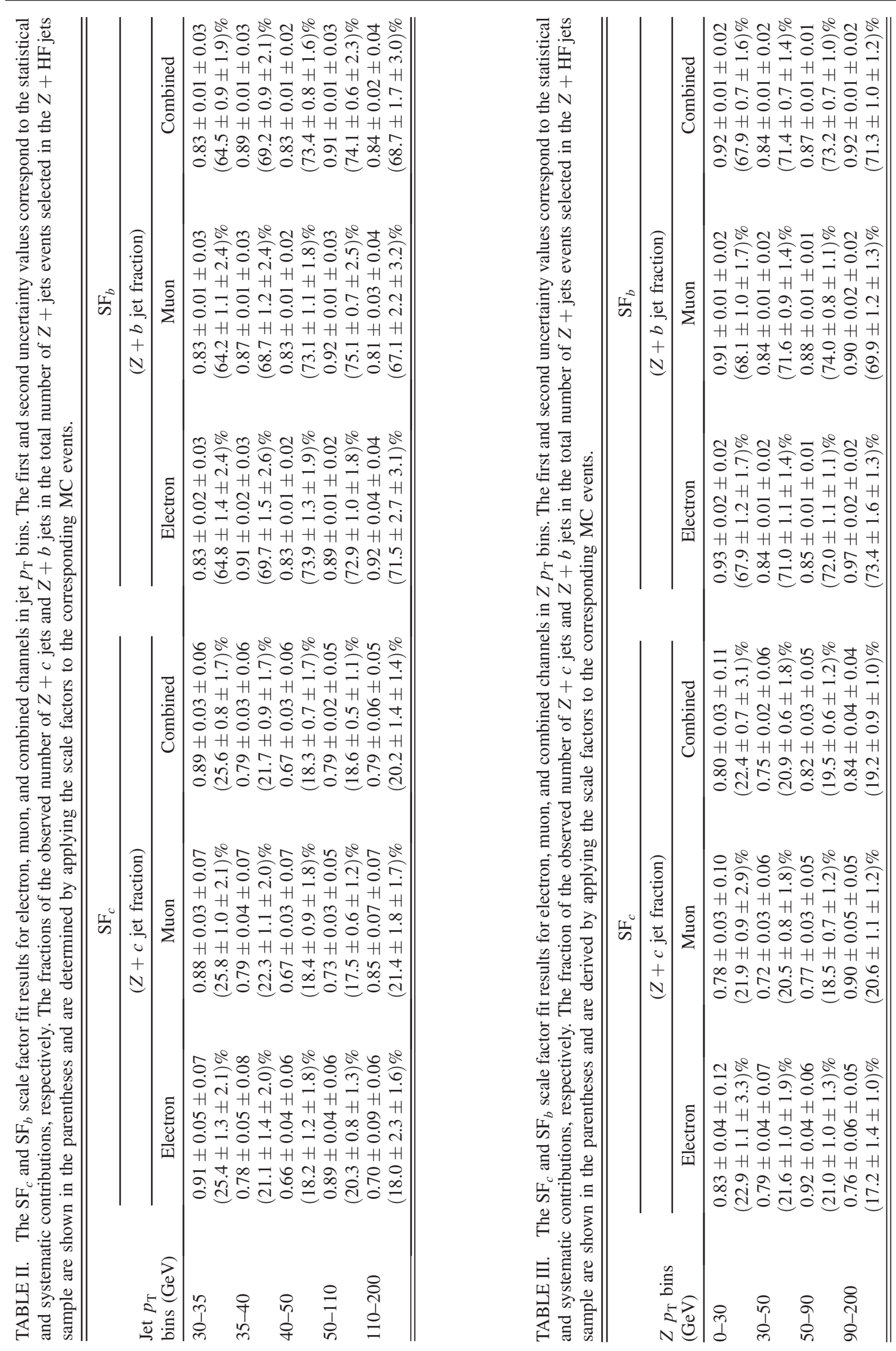


Fig. 1 (left). The pronounced enhancement seen in the $c$ jets $M_{\mathrm{SV}}$ distribution near $1.8 \mathrm{GeV}$ is due to charm meson decays.

The $M_{\mathrm{SV}}$ template for $b$ jets is derived from a highpurity data sample of $t \bar{t}$ events decaying to final states of $e \mu+\geq 2$ jets with at least one b-tagged jet. Leptons must pass similar requirements as those used in the selection of $Z+$ jets events except for a tighter isolation criterion (other activity with less than $15 \%$ of the lepton transverse momentum, instead of 25\%) to strongly suppress multijet and $W+$ jets backgrounds. The $M_{\mathrm{SV}}$ shape depends on the kinematic distributions of the corresponding jets, therefore the $b$ jet $M_{\mathrm{SV}}$ templates obtained with the $t \bar{t}$ data are corrected to account for the difference between the $b$ jet $p_{\mathrm{T}}$ spectra in $t \bar{t}$ and $Z+b$ jets events. This correction is derived from simulation by comparing the $b$ jet $M_{\mathrm{SV}}$ shapes in those two samples of events. It is parametrized as a second-order polynomial function of $M_{\mathrm{SV}}$ and varies between 3 and $20 \%$ across jet $p_{\mathrm{T}}$ ranges. A comparison between the simulated and data-driven $b$ jet $M_{\mathrm{SV}}$ distributions are presented in Fig. 1 (right). This correction procedure is applied in both the inclusive and differential measurements.

The $M_{\mathrm{SV}}$ modeling of light jets in simulation is checked in the validation sample containing $W+$ jets events selected by requiring a well-identified and isolated muon together with at least one jet. Discrimination criteria of $c$ jet versus light jets are applied, resulting in a sample with light jet purity of $\approx 40 \%$. The light jet $M_{\mathrm{SV}}$ templates in data are derived from the validation sample after subtracting nonlight jet components, which mainly consist of the $W+c$ jets events. Good agreement between the data-driven template shape and the simulation is observed.

The scale factors obtained from the combined fit in the inclusive $Z+\mathrm{HF}$ jets data sample are $\mathrm{SF}_{\mathrm{c}}=0.849 \pm$ 0.013 (stat) \pm 0.064 (syst) and $\mathrm{SF}_{\mathrm{b}}=0.873 \pm 0.005$ (stat) \pm 0.013 (syst). Tables II and III list the scale factors estimated in the jet and $Z p_{\mathrm{T}}$ bins. Details on the evaluations of systematic uncertainties in the scale factors are discussed in Section VII. The two channels pass a $\chi^{2}$ based compatibility check except for the $\mathrm{SF}_{c}$ fluctuation in one jet $p_{\mathrm{T}}$ bin of $50-110 \mathrm{GeV}$ with a $p$-value of $\approx 0.3 \%$. The post-fit $M_{\mathrm{SV}}$ distributions are shown in Fig. 2 for the measurements using the inclusive $Z+$ HF jets samples. Examples of the post-fit $M_{\mathrm{SV}}$ distributions in the muon channel for exclusive jet $p_{\mathrm{T}}$ bins are given in Appendix A, Fig. 6.

\section{UNFOLDING}

The unfolding procedure corrects the measured cross section ratios for effects related to the detector response and the event reconstruction procedures, which can lead to migrations between bins and therefore alter the true distributions. The bin-by-bin migrations are corrected by the response matrices, which quantify the migration probability between the measured and true values of a given observable (jet or $Z p_{\mathrm{T}}$ ). These matrices are derived in simulation by comparing the final-state objects (jets and leptons) at the prereconstruction ("MC-particle") and reconstruction levels.

At the MC-particle level (denoted as "particle level"), leptons are stable particles from $Z$ boson decays, dressed by adding the momenta of all photons within $\Delta R<0.1$ around the lepton directions. The particle-level jets are formed from stable particles $(\mathrm{c} \tau>1 \mathrm{~cm})$, except neutrinos, and overlapping leptons from $Z$ boson decays, using the same anti- $k_{\mathrm{T}}$ jet algorithm used for reconstructed jets.

The $Z$ boson mass and $p_{\mathrm{T}}$ at the particle level are calculated using the two leptons originating from this boson. The fiducial volume is defined by the particlelevel leptons and jets with the same kinematic requirements $\left(p_{\mathrm{T}}, \eta\right.$, and dilepton invariant mass) used in the measurement.

The response matrix is constructed using $\mathrm{MC} Z+$ jets samples. The reconstructed jets and a pair of electrons or muons are spatially matched to the corresponding particle-level objects by requiring that they are within $\Delta R<0.2$. In addition, the flavor of the reconstructed jets and the matched particle-level jets must be the same. Events that have reconstructed objects without matched particle-level objects are included in the background category and are subtracted from the sample. The acceptance and efficiency corrections account for other events that have particle-level objects in the fiducial volume but no matching reconstructed objects.

For the inclusive measurement, the acceptance corrections are derived from simulation and defined as the ratios between the number of selected events at the reconstruction level and the number of generated events within the fiducial volume. These acceptance correction factors, which depend on the jet flavor, are applied to the measured cross section ratios.

To unfold the differential distributions, the TUNFOLD package [45], which is based on a least-square fit, is used. The unfolding procedure, which solves for a wellconditioned unfolding problem in this case, is performed without regularization to avoid potential biases toward MC spectra. The data distributions of $Z+c$ jets, $Z+b$ jets, and $Z+$ jets are unfolded simultaneously to include the correlations between the denominator and numerator when deriving the unfolded ratios. The numbers of bins in the unfolded distributions are about half of those used in data to maintain the stability of the unfolding procedure. The combined response matrix used in the simultaneous unfolding is constructed from individual jet category matrices. The TUNFOLD package provides unfolded distributions together with a covariance matrix, which is used to estimate the uncertainties 

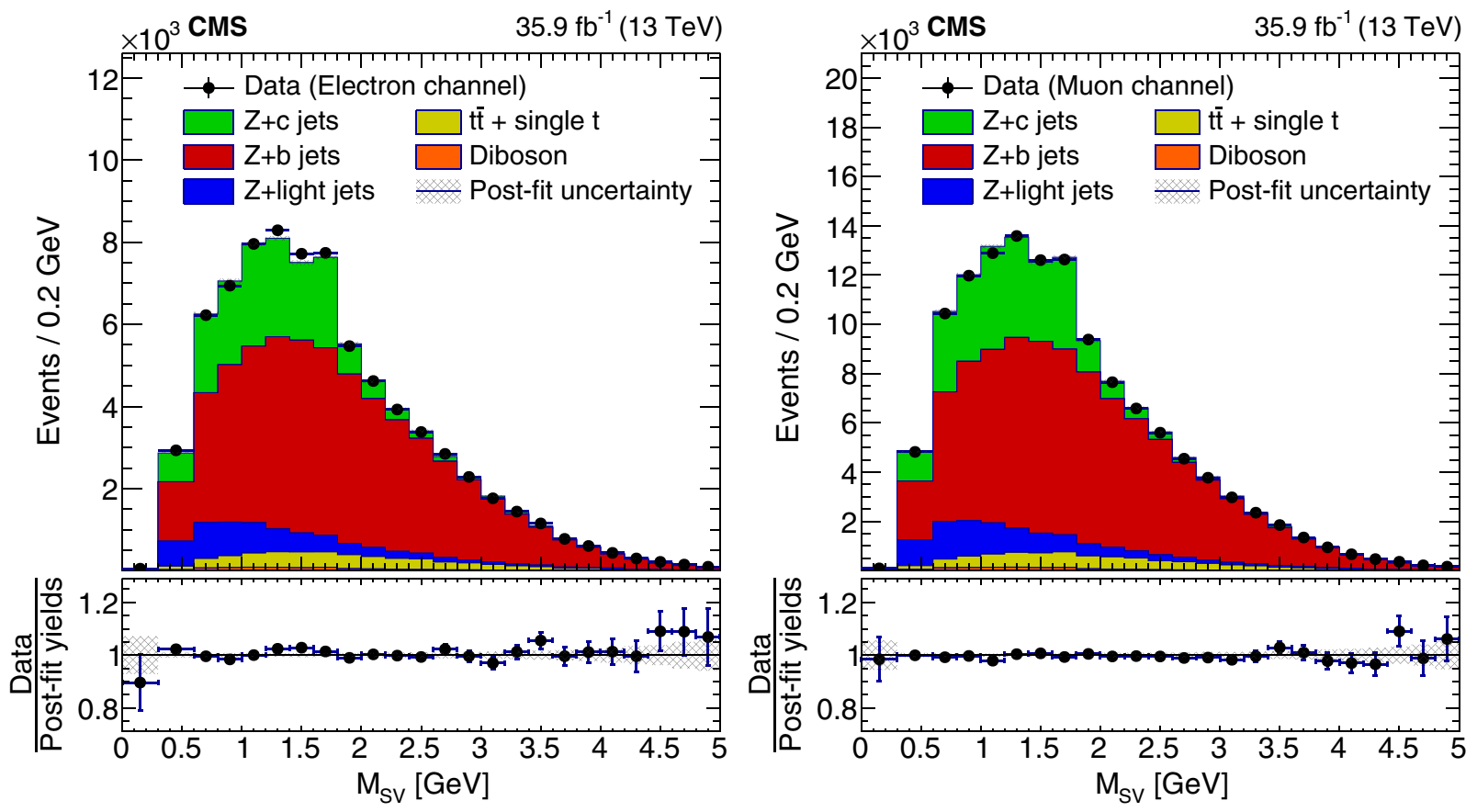

FIG. 2. Secondary vertex invariant mass distributions for the electron (left) and muon (right) channels derived from fits using the inclusive $Z+$ HF jets data sample. The post-fit uncertainty bands indicate the total uncertainties, added in quadrature, of the best-fit values of signal and background process rates.

in the unfolded cross section ratios. The unfolding procedure is checked with closure tests and bias studies using MC samples. In the closure test, response matrices are derived using one-half of the sample and the unfolding is performed on the other half. Within statistical uncertainties, the unfolded and MC truth distributions agree with each other. In the bias studies, a pull distribution is constructed by performing the unfolding on a set of $\approx 100$ MC sub-samples. The unfolding procedure converges and shows no bias.

\section{SYSTEMATIC UNCERTAINTIES}

The systematic uncertainties include experimental sources that affect the shape or normalization of templates in the scale factor fits, and the heavy-flavor tagging efficiencies. The unfolded results contain additional uncertainties related to the unfolding procedure. The following systematic uncertainties are considered in the analysis:

Jet energy scale and resolution correction: The reconstructed jet energy is corrected using a factorized model to compensate for the nonlinear and nonuniform response in the calorimeters as detailed in Ref. [39]. Since the JER is different in data and simulation, the jet energy in simulation is spread to match the resolution observed in data. Both the JES and JER corrections affect the shape of $M_{\mathrm{SV}}$ distributions used in the scale factor fits. Therefore, they contribute to the uncertainties in the $Z+c$ and $Z+b$ jets event yields.
Pileup weighting: The distribution of the number of pileup events in simulation is weighted to match that in data. The corresponding uncertainty is estimated by varying the total $p p$ inelastic cross section by $4.6 \%$ based on the measurement described in Ref. [46]. Since the shapes of $M_{\mathrm{SV}}$ templates are affected by the pileup weighting, this uncertainty source contributes to the $Z+c$ jets and $Z+b$ jets event yields as well.

Gluon splitting: Particles from a pair of collimated $c$ or $b$ quarks may end up in the same reconstructed jet, which can affect the shape of $M_{\mathrm{SV}}$ template. To quantify the corresponding uncertainties in the scale factor fit, the fraction of MC events with gluon splitting is varied by $50 \%$, which is about three times the experimental uncertainty in the gluon splitting rate measured at LEP [47,48]. The resulting variations in $\mathrm{MC} M_{\mathrm{SV}}$ shape is propagated to the scale factor fit.

Background rates: The $t \bar{t}$, single top quark, and diboson backgrounds are estimated in simulation using NNLO and NLO cross sections to normalize the event rates. The uncertainties in the $t \bar{t}$ and diboson background contributions are obtained by varying their production cross sections by 5.5 and $3.2 \%$, respectively. The uncertainty in the single top backgrounds is ignored because these backgrounds represent a very small fraction $(<1 \%)$ of the total event sample.

Statistical uncertainties of $M_{\mathrm{SV}}$ templates: A systematic uncertainty is associated to the limited number of events in the MC samples used to define the template shapes. 
TABLE IV. Systematic uncertainties in the scale factor measurements. The uncertainty ranges correspond to variations across jet and $Z p_{\mathrm{T}}$ bins.

\begin{tabular}{lcc}
\hline \hline & $\mathrm{SF}_{c}$ & $\mathrm{SF}_{b}$ \\
\hline JES, JER & $1.7-7.4 \%$ & $0.3-2.1 \%$ \\
Template statistics & $2.4-6.1 \%$ & $0.6-2.7 \%$ \\
Gluon splitting & $2.2-3.9 \%$ & $0.5-2.0 \%$ \\
Pileup weighting & $1.6-2.8 \%$ & $0.3-1.0 \%$ \\
Background uncertainty & $0.3-1.0 \%$ & $0.4-1.2 \%$ \\
$b$ jet $M_{\mathrm{SV}}$ correction & $0.2-1.6 \%$ & $0.2-0.8 \%$ \\
Total systematic uncertainty & $4.8-12.5 \%$ & $1.1-4.9 \%$ \\
\hline \hline
\end{tabular}

To estimate the corresponding uncertainty, an ensemble of the $M_{\mathrm{SV}}$ templates has been created where the bin contents have been modified by additional statistical fluctuations.

Correction of the $b$ jet $M_{\mathrm{Sv}}$ template: This systematic uncertainty is related to the ad hoc shape correction function used to derive the $b$ jet $M_{\mathrm{SV}}$ template from control samples in data. This correction, parametrized as a second order polynomial, accounts for the difference in shape of $M_{\mathrm{SV}}$ distributions in $t \bar{t}$ and $Z+$ jets events. The uncertainty of the shape correction is estimated by changing the polynomial functional forms.

Heavy-flavor tagging efficiency: The HF tagging efficiencies for $c$ and $b$ jets are estimated in simulation and corrected by the efficiency scale factors as described in Sec. VB. The systematic uncertainties of the efficiency scale factors of $c$ jets and $b$ jets with $30<p_{\mathrm{T}}<100 \mathrm{GeV}$ and $|\eta|<2.4$ are $\approx 3.5 \%$ and $\approx 1.4 \%$, respectively [42].

Missing transverse momentum selection efficiency: This uncertainty source accounts for possible differences in the $p_{\mathrm{T}}^{\text {miss }}$ selection $\left(p_{\mathrm{T}}^{\text {miss }}<40 \mathrm{GeV}\right)$ efficiencies for

TABLE V. The systematic uncertainties in the cross section ratio measurements. The uncertainty ranges correspond to variations across jet and $Z p_{\mathrm{T}}$ bins.

\begin{tabular}{lccc}
\hline \hline & $\mathrm{R}(\mathrm{c} / \mathrm{j})$ & $\mathrm{R}(\mathrm{b} / \mathrm{j})$ & $\mathrm{R}(\mathrm{c} / \mathrm{b})$ \\
\hline Scale factor measurement & $5.4-13.8 \%$ & $1.4-4.4 \%$ & $5.6-12.6 \%$ \\
HF tagging & $3.8-4.6 \%$ & $1.1-1.5 \%$ & $4.9-6.1 \%$ \\
Statistical uncertainty & $1.6-7.5 \%$ & $0.6-3.0 \%$ & $1.8-8.6 \%$ \\
\hline \hline
\end{tabular}

$Z+$ jets and $Z+$ HF jets events. The effect comes from contributions of semileptonic decays of HF hadrons in $Z+\mathrm{HF}$ jets events, which results in large $p_{\mathrm{T}}^{\text {miss }}$ values. Therefore, the efficiencies tend to be lower for $Z+$ HF jets events by $\approx 1 \%$ at high jet and $Z$ boson $p_{\mathrm{T}}$ regions compared to those of $Z+$ jets events. An uncertainty of $1.5 \%$ is included in the $\mathrm{R}(\mathrm{c} / \mathrm{j})$ and $\mathrm{R}(\mathrm{b} / \mathrm{j})$ differential results for jet ( $Z$ boson) $p_{\mathrm{T}}$ bins where $p_{\mathrm{T}}>60$ (90) GeV.

PDF and $\mu_{\mathrm{R}}, \mu_{\mathrm{F}}$ scale uncertainties: These uncertainty sources affect the unfolding correction described in Sec. VI, which is based on the $Z+$ jets MC samples. The unfolding is performed with different PDF replicas and alternative choices of the renormalization and factorization scales. The uncertainties are obtained from variations of the unfolded results and they are less than $2.5 \%, 2.8 \%$, and $2.9 \%$ in all jet and $Z p_{\mathrm{T}}$ bins for $\mathrm{R}(\mathrm{c} / \mathrm{j}), \mathrm{R}(\mathrm{b} / \mathrm{j})$, and $\mathrm{R}(\mathrm{c} / \mathrm{b})$, respectively.

Parton shower and hadronization model: The unfolding procedure is based on response matrices constructed from the $Z+$ jets simulation sample described in Sec. III. This sample uses PYTHIA to simulate the parton shower and hadronization. An alternative model is provided by the HERWIG++ generator [49]. The uncertainties in parton shower and hadronization modeling are estimated by comparing the unfolded results using response matrices from those two models. They are less than $3 \%$ for all differential cross section ratios.

Table IV summarizes the effects of systematic uncertainty sources on the $\mathrm{SF}_{c}$ and $\mathrm{SF}_{b}$ shown in Tables II and III. They are quantified as the differences in quadrature between scale factor uncertainties obtained in two fits: the nominal one where all parameters are allowed to float, and an alternative fit where the nuisance parameter corresponding to the uncertainty source of interest is fixed. The uncertainties from the scale factors and HF tagging efficiency together with the statistical uncertainties of the cross section ratios are listed in Table V.

In the unfolded differential results, the uncertainties of the measurements described here are included in the data covariance matrix, which is used to build a least squares fit of the unfolding. An error covariance matrix for the unfolded distributions is estimated. This includes the uncertainties from the data, response matrix, and the unfolding procedure.

TABLE VI. Cross section ratios measured in the electron and muon channels, along with the combined results. The first and second uncertainty values correspond to the statistical and systematic contributions, respectively.

\begin{tabular}{lccc}
\hline \hline & Electron & Muon & Combined \\
\hline $\mathrm{R}(\mathrm{c} / \mathrm{j})$ & $0.098 \pm 0.002 \pm 0.009$ & $0.094 \pm 0.002 \pm 0.008$ & $0.095 \pm 0.002 \pm 0.008$ \\
$\mathrm{R}(\mathrm{b} / \mathrm{j})$ & $0.0546 \pm 0.0005 \pm 0.0010$ & $0.0538 \pm 0.0004 \pm 0.0010$ & $0.0541 \pm 0.0003 \pm 0.0011$ \\
$\mathrm{R}(\mathrm{c} / \mathrm{b})$ & $1.80 \pm 0.05 \pm 0.17$ & $1.75 \pm 0.04 \pm 0.16$ & $1.76 \pm 0.03 \pm 0.16$ \\
\hline \hline
\end{tabular}


TABLE VII. Unfolded cross section ratios in the electron and muon channels, along with the combined results. The first and second uncertainty values correspond to the statistical and systematic contributions, respectively.

\begin{tabular}{lccc}
\hline \hline & Electron & Muon & Combined \\
\hline $\mathrm{R}(\mathrm{c} / \mathrm{j})$ & $0.105 \pm 0.003 \pm 0.009$ & $0.101 \pm 0.002 \pm 0.009$ & $0.102 \pm 0.002 \pm 0.009$ \\
$\mathrm{R}(\mathrm{b} / \mathrm{j})$ & $0.0639 \pm 0.0006 \pm 0.0015$ & $0.0629 \pm 0.0005 \pm 0.0014$ & $0.0633 \pm 0.0004 \pm 0.0015$ \\
$\mathrm{R}(\mathrm{c} / \mathrm{b})$ & $1.65 \pm 0.04 \pm 0.15$ & $1.61 \pm 0.04 \pm 0.15$ & $1.62 \pm 0.03 \pm 0.15$ \\
\hline \hline
\end{tabular}

\section{RESULTS}

The observed and corrected (for the acceptance and efficiency) cross section ratios for the inclusive measurements are summarized in Tables VI and VII, respectively. The measured differential cross section ratios are presented in the Appendix B, Tables IX and X.

The unfolded differential cross section ratios, $\mathrm{R}(\mathrm{c} / \mathrm{j})$, $\mathrm{R}(\mathrm{b} / \mathrm{j})$, and $\mathrm{R}(\mathrm{c} / \mathrm{b})$, versus the jet and $Z$ boson $p_{\mathrm{T}}$ are shown in Figs. 3, 4, and 5, respectively. The results are compared with predictions from the MG5_aMC and MCFM programs [50-52], both at LO and NLO. The renormalization and factorization scales in the matrix element and the PDF uncertainties are included in these predictions. For the former, the scales are varied between
0.5 and 2 times their nominal value such that the $\mu_{\mathrm{R}} / \mu_{\mathrm{F}}$ ratio is kept between 0.5 and 2. This conventional choice is implemented in the CMS-default settings for generating samples to estimate the theoretical MG5_aMC LO and NLO cross sections. The uncertainty due to the scales is taken to be the envelope of these predictions. In addition, for the MCFM calculation, the constraint on the $\mu_{\mathrm{R}} / \mu_{\mathrm{F}}$ ratio is dropped, i.e., the scales are varied independently. This more conservative choice is motivated by the fact that the $Z+\mathrm{HF}$ jets cross sections as functions of the renormalization and factorization scales have opposite trends [1]. The MCFM error bands in Figs. 3-5 correspond to this choice of scale variation. The uncertainties due to the scales in the cross section
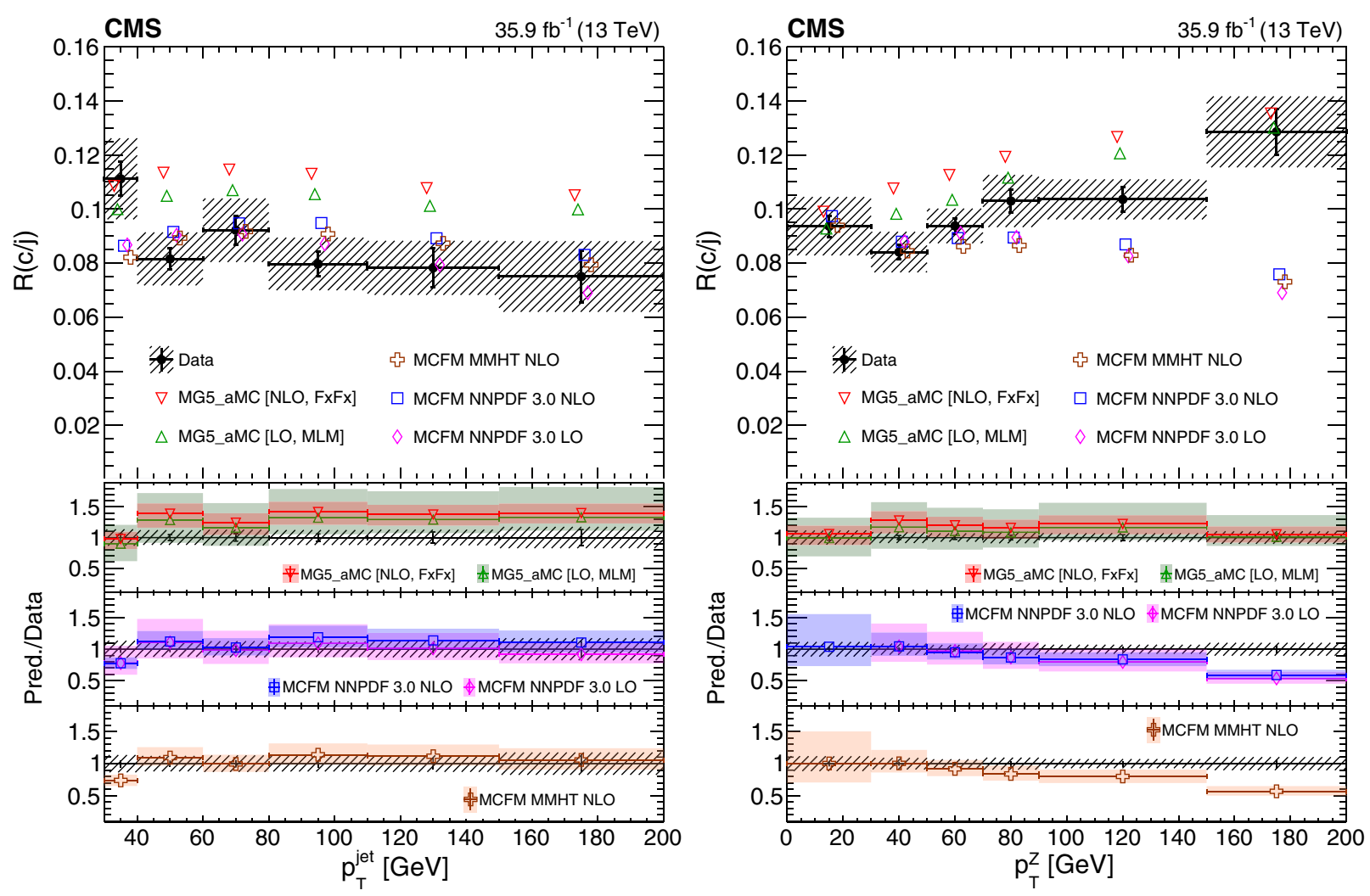

FIG. 3. Unfolded, particle-level MG5_amC, and parton-level MCFM R(c/j) cross section ratios versus jet (left) and $Z$ boson (right) transverse momentum. The vertical error bars for the data points are statistical while the hatched band represents the total uncertainties. The predictions are slightly shifted along the $x$-axis for readability in the upper plots, and their total PDF and scale uncertainties are shown as error bands in the ratio plots. 

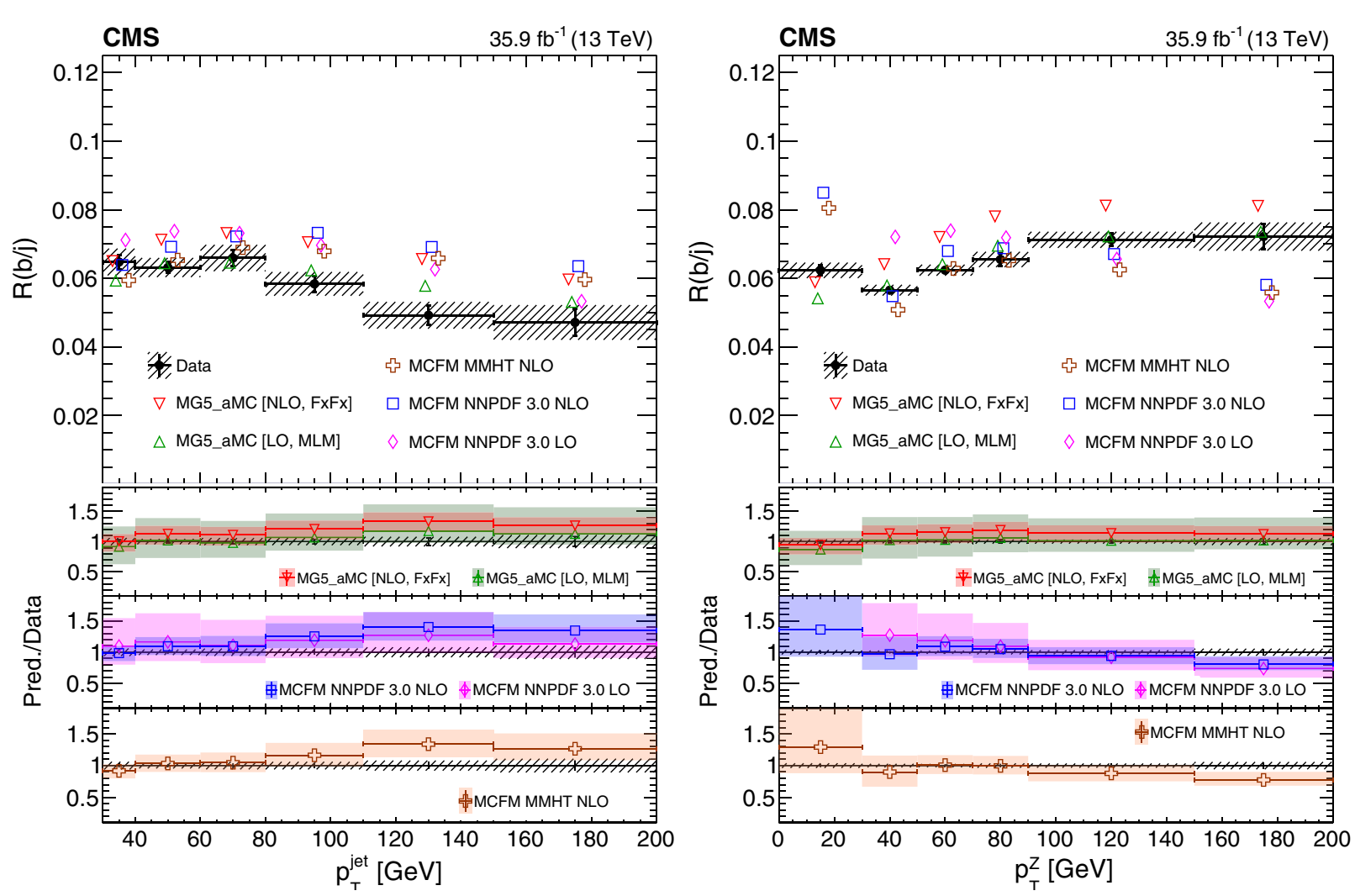

FIG. 4. Unfolded, particle-level MG5_aMC, and parton-level MCFM R(b/j) cross section ratio versus jet (left) and $Z$ boson (right) transverse momentum. The vertical error bars for the data points are statistical while the hatched band presents the total uncertainties. The predictions are slightly shifted along the $x$-axis for readability in the upper plots, and their total PDF and scale uncertainties are shown as error bands in the ratio plots.

ratios are obtained by adding uncertainties in the numerator and denominator in quadrature, i.e., they are assumed to be uncorrelated. The PDF uncertainty is evaluated by changing the replicas of the PDF set.

The LO cross sections are computed using MG5_aMC interfaced with PYTHIA through the $k_{\mathrm{T}}$-MLM matching scheme [53,54]. The LO matrix element calculations include processes with up to 4 outgoing partons. The NNPDF 3.0 LO PDF set is used and the matching scale together with the strong coupling constant $\alpha_{\mathrm{S}}$ at the $Z$ boson mass are set at $19 \mathrm{GeV}$ and 0.130 , respectively. The multileg MG5_aMC generator interfaced with PYTHIA using the FxFx matching scheme evaluates the cross section ratios at NLO precision. The choice of parameters is described in Sec. III.

The MCFM generator is used to perform calculations of the cross sections and cross section ratios at the parton level in the 5FS. The $Z+$ jets cross sections are evaluated by a simple cone algorithm with a radius of 0.4 (i.e., partons are merged if the distances, $\Delta R$, between them are less than 0.4). The central values for the cross sections are evaluated at $\mu_{\mathrm{R}}$ and $\mu_{\mathrm{F}}$ set to the mass of the $Z$ boson. In addition, the NLO MCFM results are shown for two PDFs, NNPDF 3.0 and
MMHT14 [55], along with the MCFM LO cross section ratios. The values of $\alpha_{\mathrm{S}}$ are taken from those PDFs. Table VIII shows the predicted inclusive cross section ratios from MG5_aMC and MCFM.

A few comments are in order when comparing data with various predictions. The MG5_aMC predictions for the cross section ratios are higher in most of the bins, although still compatible with the data given the large uncertainties, except for the $\mathrm{R}(\mathrm{c} / \mathrm{j})$ versus jet $p_{\mathrm{T}}$, where the deviations are more pronounced. The data are better described with MG5_aMC at LO compared to MG5_aMC at NLO. These observations are similar to those reported in previous measurements at $8 \mathrm{TeV}[11,56]$. The MCFM predictions for $\mathrm{R}(\mathrm{c} / \mathrm{j})$ and $\mathrm{R}(\mathrm{b} / \mathrm{j})$ disagree with data at high jet and $Z$ $p_{\mathrm{T}}$, except for $\mathrm{R}(\mathrm{c} / \mathrm{j})$ versus jet $p_{\mathrm{T}}$, where in general there is good agreement with LO or NLO calculations, and for both PDFs considered. For R(c/b), however, all theoretical predictions are consistent with the measured ratios, except for the MCFM prediction for the highest $Z$ boson $p_{\mathrm{T}}$ bin. The difference between the parton- and particle-level jets may affect the MCFM predictions, although the corresponding effects are significantly reduced or vanish in the cross section ratios. Alternatively, higher order pQCD calculations might be needed to describe the data. 

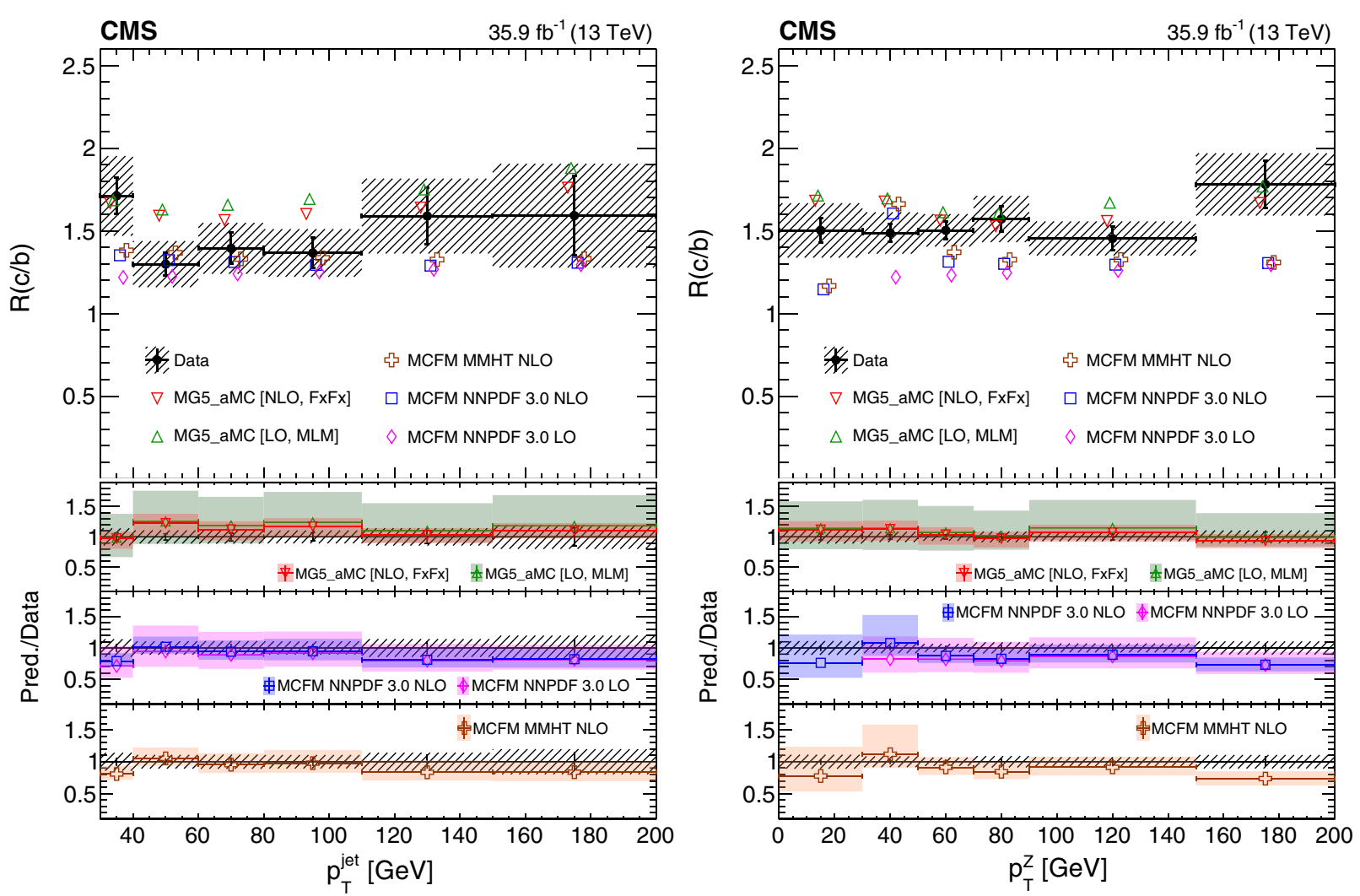

FIG. 5. Unfolded, particle-level MG5_aMC, and parton-level MCFM R(c/b) cross section ratio versus jet (left) and $Z$ boson (right) transverse momentum. The vertical error bars for the data points are statistical while the hatched band represents the total uncertainties. The predictions are slightly shifted along the $x$-axis for readability in the upper plots, and their total PDF and scale uncertainties are shown as error bands in the ratio plots.

\section{SUMMARY}

Ratios of cross sections, $\sigma(Z+c$ jets $) / \sigma(Z+$ jets $)$, $\sigma(Z+b$ jets $) / \sigma(Z+$ jets $)$, and $\sigma(Z+c$ jets $) / \sigma(Z+b$ jets $)$ in the associated production of a $Z$ boson with at least one charm or bottom quark jet have been measured in proton-proton collisions at $\sqrt{s}=13 \mathrm{TeV}$ using $35.9 \mathrm{fb}^{-1}$ of data collected by the CMS experiment at the LHC. The fiducial volume of the measurement is defined by $p_{\mathrm{T}}>30 \mathrm{GeV}$ and $|\eta|<2.4$ for the jets, where $p_{\mathrm{T}}$ and $\eta$ represent transverse momentum and pseudorapidity, respectively. The $Z$ bosons are selected within the mass range of 71 and $111 \mathrm{GeV}$ requiring leptons (electrons or muons) with $p_{\mathrm{T}}>25 \mathrm{GeV}$ and $|\eta|<2.4$. The measured values are $\sigma(Z+c$ jets $) /$ $\sigma(Z+$ jets $)=0.102 \pm 0.002 \pm 0.009, \quad \sigma(Z+b$ jets $) /$ $\sigma(Z+$ jets $)=0.0633 \pm 0.0004 \pm 0.0015$, and $\sigma(Z+$ $c$ jets $) / \sigma(Z+b$ jets $)=1.62 \pm 0.03 \pm 0.15$. Results for the inclusive and differential cross section ratios as functions of jet and $Z$ boson transverse momentum are compared with predictions from leading and next-toleading order perturbative quantum chromodynamics calculations. These are the first results of this kind at $13 \mathrm{TeV}$.

TABLE VIII. Predicted cross section ratios from MG5_aMC and MCFM at LO and NLO accuracy. The first and second sets of uncertainties correspond to PDF and scale variations, respectively. The scale uncertainties for MCFM with $\mu_{\mathrm{R}} / \mu_{\mathrm{F}}$ ratio kept between 0.5 and 2 are in the parentheses.

\begin{tabular}{lcccc}
\hline \hline & MG5_aMC (NLO, FxFx) & MCFM (NLO) & MG5_aMC (LO, MLM) & MCFM (LO) \\
\hline $\mathrm{R}(\mathrm{c} / \mathrm{j})$ & $0.111 \pm 0.003_{-0.011}^{+0.010}$ & $0.090 \pm 0.003_{-0.012}^{+0.010}\left({ }_{-0.007}^{+0.008}\right)$ & $0.103 \pm 0.003_{-0.026}^{+0.028}$ & $0.087 \pm 0.003_{-0.022}^{+0.025}$ \\
$\mathrm{R}(\mathrm{b} / \mathrm{j})$ & $0.067 \pm 0.002 \pm 0.006$ & $0.068 \pm 0.002_{-0.011}^{+0.008}( \pm 0.006)$ & $0.062 \pm 0.002_{-0.015}^{+0.018}$ & $0.071 \pm 0.002_{-0.021}^{+0.023}$ \\
$\mathrm{R}(\mathrm{c} / \mathrm{b})$ & $1.64 \pm 0.05_{-0.16}^{+0.15}$ & $1.33 \pm 0.04_{-0.21}^{+0.16}\left(_{-0.12}^{+0.10}\right)$ & $1.67 \pm 0.06_{-0.40}^{+0.54}$ & $1.20 \pm 0.04_{-0.38}^{+0.42}$ \\
\hline \hline
\end{tabular}




\section{ACKNOWLEDGMENTS}

We congratulate our colleagues in the CERN accelerator departments for the excellent performance of the LHC and thank the technical and administrative staffs at CERN and at other CMS institutes for their contributions to the success of the CMS effort. In addition, we gratefully acknowledge the computing centers and personnel of the Worldwide LHC Computing Grid for delivering so effectively the computing infrastructure essential to our analyses. Finally, we acknowledge the enduring support for the construction and operation of the LHC and the CMS detector provided by the following funding agencies: BMBWF and FWF (Austria); FNRS and FWO (Belgium); CNPq, CAPES, FAPERJ, FAPERGS, and FAPESP (Brazil); MES (Bulgaria); CERN; CAS, MoST, and NSFC (China); COLCIENCIAS (Colombia); MSES and CSF (Croatia); RPF (Cyprus); SENESCYT (Ecuador); MoER, ERC IUT, PUT and ERDF (Estonia); Academy of Finland, MEC, and HIP (Finland); CEA and CNRS/IN2P3 (France); BMBF, DFG, and HGF (Germany); GSRT (Greece); NKFIA (Hungary); DAE and DST (India); IPM (Iran); SFI (Ireland); INFN (Italy); MSIP and NRF (Republic of Korea); MES (Latvia); LAS (Lithuania); MOE and UM (Malaysia); BUAP, CINVESTAV, CONACYT, LNS, SEP, and UASLP-FAI (Mexico); MOS (Montenegro); MBIE (New Zealand); PAEC (Pakistan); MSHE and NSC (Poland); FCT (Portugal); JINR (Dubna); MON, RosAtom, RAS, RFBR, and NRC KI (Russia); MESTD (Serbia); SEIDI, CPAN, PCTI, and FEDER (Spain); MOSTR (Sri Lanka); Swiss Funding Agencies (Switzerland); MST (Taipei); ThEPCenter, IPST, STAR, and NSTDA (Thailand); TUBITAK and TAEK (Turkey); NASU (Ukraine); STFC (United Kingdom); DOE and NSF (USA). Individuals have received support from the MarieCurie program and the European Research Council and Horizon 2020 Grant, Contracts No. 675440, No. 752730, and No. 765710 (European Union); the Leventis Foundation; the A.P. Sloan Foundation; the Alexander von Humboldt Foundation; the Belgian Federal Science
Policy Office; the Fonds pour la Formation à la Recherche dans l'Industrie et dans l'Agriculture (FRIA-Belgium); the Agentschap voor Innovatie door Wetenschap en Technologie (IWT-Belgium); the F. R. S.-FNRS and FWO (Belgium) under the "Excellence of Science-EOS"-be.h Project No. 30820817; the Beijing Municipal Science \& Technology Commission, No. Z191100007219010; the Ministry of Education, Youth and Sports (MEYS) of the Czech Republic; the Deutsche Forschungsgemeinschaft (DFG) under Germanys Excellence Strategy-EXC 2121 "Quantum Universe"-390833306; the Lendület ("Momentum") Program and the János Bolyai Research Scholarship of the Hungarian Academy of Sciences, the New National Excellence Program ÚNKP, the NKFIA research Grants No. 123842, No. 123959, No. 124845, No. 124850 , No. 125105 , No. 128713 , No. 128786 , and No. 129058 (Hungary); the Council of Science and Industrial Research, India; the HOMING PLUS program of the Foundation for Polish Science, cofinanced from European Union, Regional Development Fund, the Mobility Plus program of the Ministry of Science and Higher Education, the National Science Center (Poland), Contracts No. Harmonia 2014/14/M/ST2/00428, No. Opus 2014/13/B/ST2/02543, No. 2014/15/B/ST2/03998, and No. 2015/19/B/ST2/02861, No. Sonata-bis 2012/07/E/ ST2/01406; the National Priorities Research Program by Qatar National Research Fund; the Ministry of Science and Education, Grant No. 14.W03.31.0026 (Russia); the Programa Estatal de Fomento de la Investigación Científica y Técnica de Excelencia María de Maeztu, Grant No. MDM-2015-0509 and the Programa Severo Ochoa del Principado de Asturias; the Thalis and Aristeia programs cofinanced by EU-ESF and the Greek NSRF; the Rachadapisek Sompot Fund for Postdoctoral Fellowship, Chulalongkorn University and the Chulalongkorn Academic into Its 2nd Century Project Advancement Project (Thailand); the Kavli Foundation; the Nvidia Corporation; the SuperMicro Corporation; the Welch Foundation, Contract No. C-1845; and the Weston Havens Foundation (USA). 


\section{APPENDIX A: POSTFIT $M_{\text {SV }}$ DISTRIBUTIONS IN THE EXCLUSIVE JET $p_{\mathrm{T}}$ BINS}
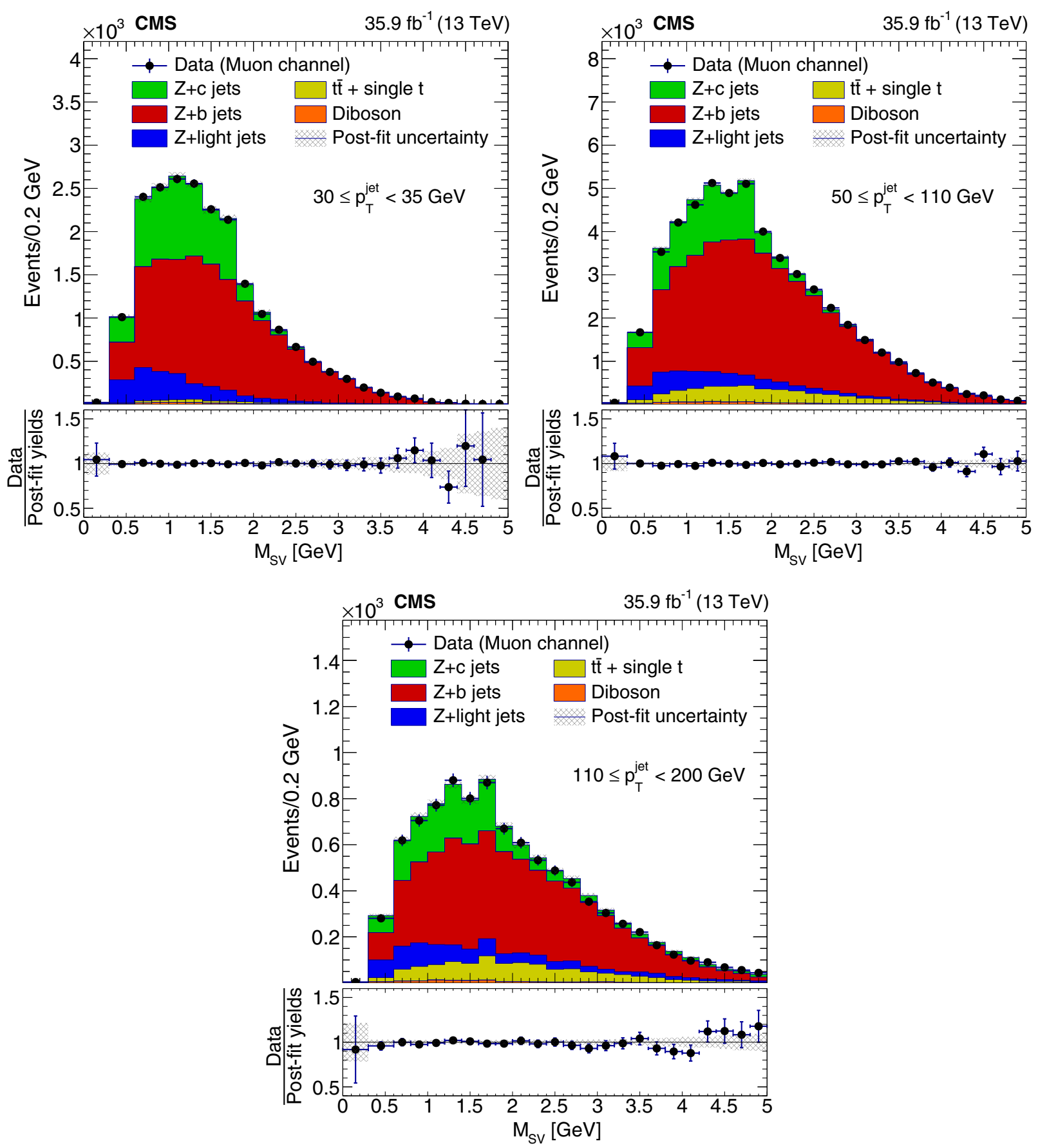

FIG. 6. Secondary vertex invariant mass distributions for jet $p_{\mathrm{T}}$ bins $30-35 \mathrm{GeV}, 50-110 \mathrm{GeV}$, and $110-200 \mathrm{GeV}$ in the muon channel derived from fits using the corresponding jet $p_{\mathrm{T}}$ binned $Z+\mathrm{HF}$ jets data samples. The postfit uncertainty bands indicate the total uncertainties, added in quadrature, of the best-fit values of signal and background process rates. 
论

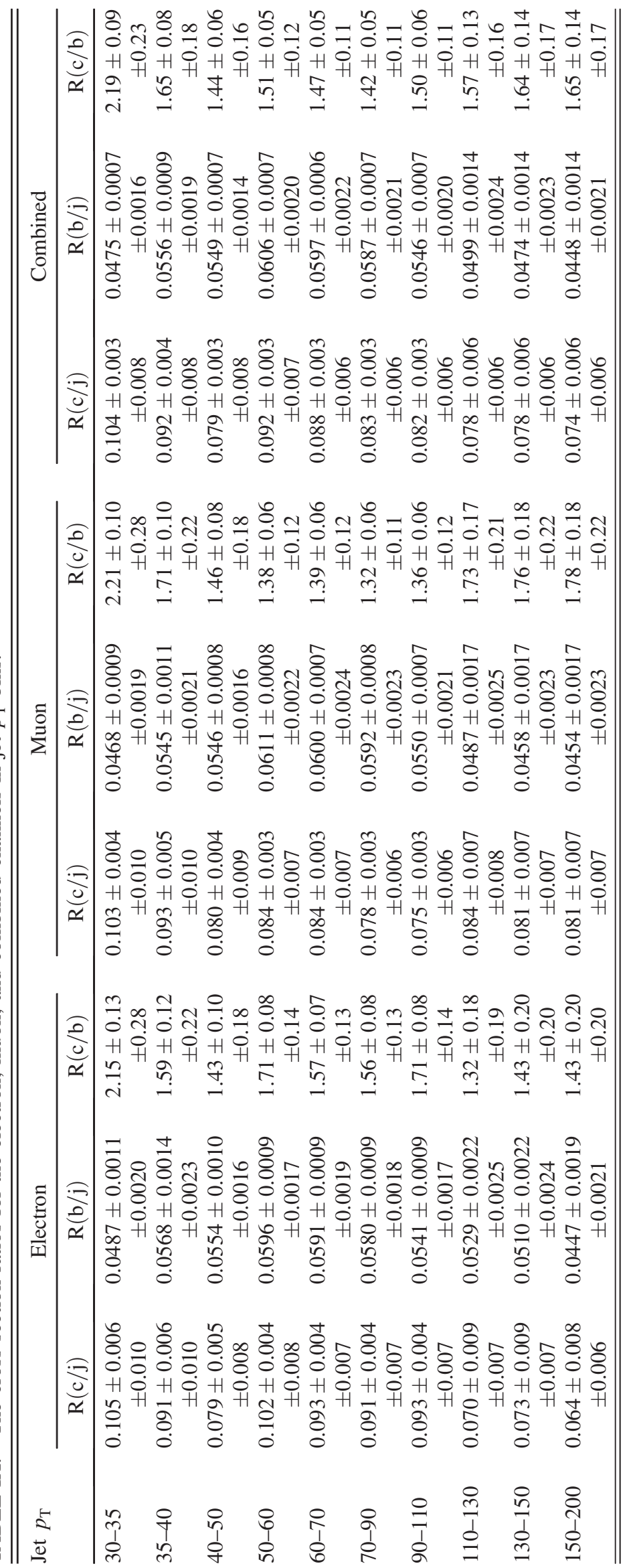




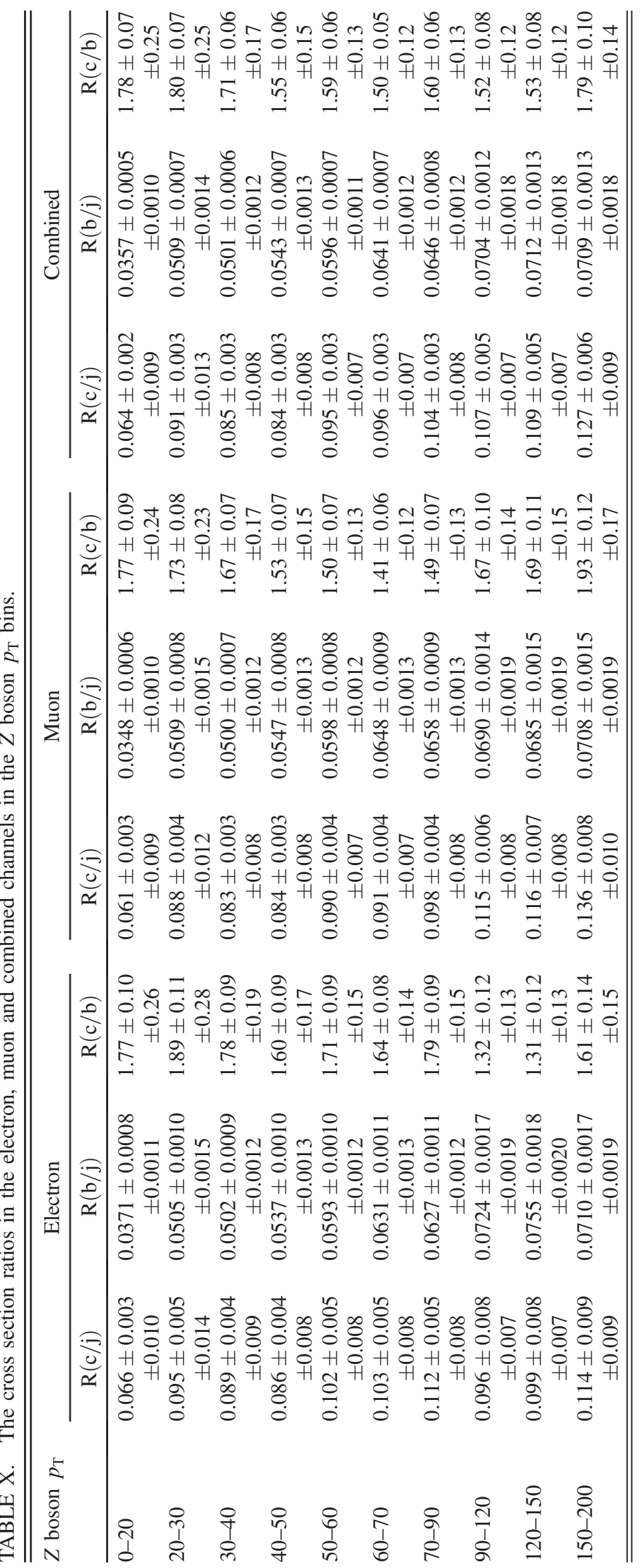


[1] J. M. Campbell, R. K. Ellis, F. Maltoni, and S. Willenbrock, Associated production of a $\mathrm{Z}$ boson and a single heavy quark jet, Phys. Rev. D 69, 074021 (2004).

[2] F. F. Cordero, L. Reina, and D. Wackeroth, W and Z boson production with a massive bottom-quark pair at the Large Hadron Collider, Phys. Rev. D 80, 034015 (2009).

[3] F. Maltoni, G. Ridolfi, and M. Ubiali, b-initiated processes at the LHC: A reappraisal, J. High Energy Phys. 07 (2012) 022 .

[4] T. Aaltonen et al. (CDF Collaboration), Measurement of cross sections for $\mathrm{b}$ jet production in events with a $\mathrm{Z}$ boson in $\mathrm{p} \overline{\mathrm{p}}$ collisions at $\sqrt{s}=1.96 \mathrm{TeV}$, Phys. Rev. D 79, 052008 (2009).

[5] V. M. Abazov et al. (D0 Collaboration), Measurement of Associated Production of Z Bosons with Charm Quark Jets in $\mathrm{p} \overline{\mathrm{p}}$ Collisions at $\sqrt{s}=1.96 \mathrm{TeV}$, Phys. Rev. Lett. 112, 042001 (2014).

[6] V. M. Abazov et al. (D0 Collaboration), Measurement of the ratio of inclusive cross sections $\sigma(\mathrm{p} \overline{\mathrm{p}} \rightarrow \mathrm{Z}+2 \mathrm{~b}$ jets $) /$ $\sigma(\mathrm{p} \overline{\mathrm{p}} \rightarrow \mathrm{Z}+2$ jets $)$ in $\mathrm{p} \overline{\mathrm{p}}$ collisions at $\sqrt{s}=1.96 \mathrm{TeV}$, Phys. Rev. D 91, 052010 (2015).

[7] ATLAS Collaboration, Measurement of differential production cross-sections for a $\mathrm{Z}$ boson in association with $\mathrm{b}$ jets in $7 \mathrm{TeV}$ proton-proton collisions with the ATLAS detector, J. High Energy Phys. 10 (2014) 141.

[8] CMS Collaboration, Measurement of the production cross sections for a $\mathrm{Z}$ boson and one or more $\mathrm{b}$ jets in pp collisions at $\sqrt{s}=7 \mathrm{TeV}$, J. High Energy Phys. 06 (2014) 120.

[9] LHCb Collaboration, Measurement of the $Z+b$-jet crosssection in pp collisions at $\sqrt{s}=7 \mathrm{TeV}$ in the forward region, J. High Energy Phys. 01 (2015) 064.

[10] LHCb Collaboration, Observation of associated production of a $\mathrm{Z}$ boson with a $\mathrm{D}$ meson in the forward region, J. High Energy Phys. 04 (2014) 091.

[11] CMS Collaboration, Measurement of associated $Z+$ charm production in proton-proton collisions at $\sqrt{s}=8 \mathrm{TeV}$, Eur. Phys. J. C 78, 287 (2018).

[12] J. Alwall, M. Herquet, F. Maltoni, O. Mattelaer, and T. Stelzer, MadGraph 5: Going beyond, J. High Energy Phys. 06 (2011) 128.

[13] J. Alwall, R. Frederix, S. Frixione, V. Hirschi, F. Maltoni, O. Mattelaer, H. S. Shao, T. Stelzer, P. Torrielli, and M. Zaro, The automated computation of tree-level and next-toleading order differential cross sections, and their matching to parton shower simulations, J. High Energy Phys. 07 (2014) 079.

[14] CMS Collaboration, The CMS trigger system, J. Instrum. 12, P01020 (2017).

[15] CMS Collaboration, The CMS experiment at the CERN LHC, J. Instrum. 3, S08004 (2008).

[16] CMS luminosity measurements for the 2016 data taking period, CMS physics analysis summary Report No. CMSPAS-LUM-17-001, 2017, https://cds.cern.ch/record/ 2257069.

[17] S. Agostinelli et al. (GEANT4 Collaboration), GEANT4-A simulation toolkit, Nucl. Instrum. Methods Phys. Res., Sect. A 506, 250 (2003).

[18] T. Sjöstrand, S. Ask, J. R. Christiansen, R. Corke, N. Desai, P. Ilten, S. Mrenna, S. Prestel, C. O. Rasmussen, and P.Z.
Skands, An introduction to PYTHIA 8.2, Comput. Phys. Commun. 191, 159 (2015).

[19] R. Frederix and S. Frixione, Merging meets matching in MC@NLO, J. High Energy Phys. 12 (2012) 061.

[20] K. Melnikov and F. Petriello, Electroweak gauge boson production at hadron colliders through $\mathcal{O}\left(\alpha_{S}^{2}\right)$, Phys. Rev. D 74, 114017 (2006).

[21] P. Nason, A new method for combining NLO QCD with shower Monte Carlo algorithms, J. High Energy Phys. 11 (2004) 040.

[22] S. Frixione, P. Nason, and C. Oleari, Matching NLO QCD computations with parton shower simulations: The POWHEG method, J. High Energy Phys. 11 (2007) 070.

[23] S. Alioli, P. Nason, C. Oleari, and E. Re, A general framework for implementing NLO calculations in shower Monte Carlo programs: The POWHEG BOX, J. High Energy Phys. 06 (2010) 043.

[24] S. Frixione, P. Nason, and G. Ridolfi, A positive-weight next-to-leading-order Monte Carlo for heavy flavour hadroproduction, J. High Energy Phys. 09 (2007) 126.

[25] M. Czakon and A. Mitov, Top++: A program for the calculation of the top-pair cross-section at hadron colliders, Comput. Phys. Commun. 185, 2930 (2014).

[26] M. Grazzini, S. Kallweit, and M. Wiesemann, Fully differential NNLO computations with MATRIX, Eur. Phys. J. C 78, 537 (2018).

[27] N. Kidonakis, Top quark production, in Proceedings, Helmholtz International Summer School on Physics of Heavy Quarks and Hadrons (HQ 2013): JINR, Dubna, Russia, 2013 (DESY: Verlag Deutsches ElektronenSynchotron, Hamburg, 2014), http://dx.doi.org/10.3204/ DESY-PROC-2013-03/Kidonakis.

[28] R. D. Ball et al. (NNPDF Collaboration), Parton distributions for the LHC run II, J. High Energy Phys. 04 (2015) 040.

[29] CMS Collaboration, Event generator tunes obtained from underlying event and multiparton scattering measurements, Eur. Phys. J. C 76, 155 (2016).

[30] Investigations of the impact of the parton shower tuning in PYTHIA8 in the modelling of $t \bar{t}$ at $\sqrt{s}=8$ and $13 \mathrm{TeV}$, CMS Physics Analysis Summary CMS-PAS-TOP-16-021, 2016, https://cds.cern.ch/record/2235192.

[31] CMS Collaboration, Particle-flow reconstruction and global event description with the CMS detector, J. Instrum. 12, P10003 (2017).

[32] M. Cacciari, G. P. Salam, and G. Soyez, The anti- $k_{\mathrm{T}}$ jet clustering algorithm, J. High Energy Phys. 04 (2008) 063.

[33] M. Cacciari, G. P. Salam, and G. Soyez, FastJet user manual, Eur. Phys. J. C 72, 1896 (2012).

[34] CMS Collaboration, Technical proposal for the Phase-II upgrade of the Compact Muon Solenoid, CMS Technical proposal Report Nos. CERN-LHCC-2015-010, CMS-TDR15-02, CERN, 2015.

[35] CMS Collaboration, Performance of electron reconstruction and selection with the CMS detector in proton-proton collisions at $\sqrt{s}=8 \mathrm{TeV}$, J. Instrum. 10, P06005 (2015).

[36] CMS Collaboration, Performance of the CMS muon detector and muon reconstruction with proton-proton collisions at $\sqrt{s}=13 \mathrm{TeV}$, J. Instrum. 13, P06015 (2018). 
[37] M. Cacciari and G. P. Salam, Pileup subtraction using jet areas, Phys. Lett. B 659, 119 (2008).

[38] CMS Collaboration, Determination of jet energy calibration and transverse momentum resolution in CMS, J. Instrum. 6, P11002 (2011).

[39] CMS Collaboration, Jet energy scale and resolution in the CMS experiment in pp collisions at $8 \mathrm{TeV}$, J. Instrum. 12, P02014 (2017).

[40] Jet algorithms performance in $13 \mathrm{TeV}$ data, CMS Physics Analysis Summary Report No. CMS-PAS-JME-16-003, 2017, https://cds.cern.ch/record/2256875.

[41] CMS Collaboration, Performance of missing transverse momentum in proton-proton collisions at $\sqrt{s}=13 \mathrm{TeV}$ using the CMS detector, J. Instrum. 14, P07004 (2019).

[42] CMS Collaboration, Identification of heavy-flavour jets with the CMS detector in pp collisions at $13 \mathrm{TeV}$, J. Instrum. 13, P05011 (2018).

[43] W. Waltenberger, Adaptive vertex reconstruction, Technical Report No. CMS-NOTE-2008-033, 2008.

[44] CMS Collaboration, Identification of b-quark jets with the CMS experiment, J. Instrum. 8, P04013 (2013).

[45] S. Schmitt, TUnfold: An algorithm for correcting migration effects in high energy physics, J. Instrum. 7, T10003 (2012).

[46] CMS Collaboration, Measurement of the inelastic protonproton cross section at $\sqrt{s}=13 \mathrm{TeV}$, J. High Energy Phys. 07 (2018) 161.

[47] A. Heister et al. (ALEPH Collaboration), A measurement of the gluon splitting rate into $c \bar{c}$ pairs in hadronic $Z$ decays, Phys. Lett. B 561, 213 (2003).
[48] R. Barate et al. (ALEPH Collaboration), A measurement of the gluon splitting rate into $b \bar{b}$ pairs in hadronic $Z$ decays, Phys. Lett. B 434, 437 (1998).

[49] M. Bähr, S. Gieseke, M. A. Gigg, D. Grellscheid, K. Hamilton, O. Latunde-Dada, S. Plätzer, P. Richardson, M. H. Seymour, A. Sherstnev, and B. R. Webber, Herwig++ physics and manual, Eur. Phys. J. C 58, 639 (2008).

[50] J. M. Campbell and R. K. Ellis, Update on vector boson pair production at hadron colliders, Phys. Rev. D 60, 113006 (1999).

[51] J. M. Campbell, R. K. Ellis, and C. Williams, Vector boson pair production at the LHC, J. High Energy Phys. 07 (2011) 018.

[52] J. M. Campbell, R. K. Ellis, and W. Giele, A multi-threaded version of MCFM, Eur. Phys. J. C 75, 246 (2015).

[53] J. Alwall, S. Höche, F. Krauss, N. Lavesson, L. Lönnblad, F. Maltoni, M. L. Mangano, M. Moretti, C. G. Papadopoulos, F. Piccinini, S. Schumann, M. Treccani, J. Winter, and M. Worek, Comparative study of various algorithms for the merging of parton showers and matrix elements in hadronic collisions, Eur. Phys. J. C 53, 473 (2008).

[54] J. Alwall, S. de Visscher, and F. Maltoni, QCD radiation in the production of heavy colored particles at the LHC, J. High Energy Phys. 02 (2009) 017.

[55] L. A. Harland-Lang, A. D. Martin, P. Motylinski, and R. S. Thorne, Parton distributions in the LHC era: MMHT 2014 PDFs, Eur. Phys. J. C 75, 204 (2015).

[56] CMS Collaboration, Measurements of the associated production of a $Z$ boson and $b$ jets in $p p$ collisions at $\sqrt{s}=8 \mathrm{TeV}$, Eur. Phys. J. C 77, 751 (2017).

A. M. Sirunyan, ${ }^{1, a}$ A. Tumasyan, ${ }^{1}$ W. Adam, ${ }^{2}$ F. Ambrogi, ${ }^{2}$ T. Bergauer, ${ }^{2}$ J. Brandstetter, ${ }^{2}$ M. Dragicevic, ${ }^{2}$ J. Erö, ${ }^{2}$ A. Escalante Del Valle, ${ }^{2}$ M. Flechl, ${ }^{2}$ R. Frühwirth, ${ }^{2, b}$ M. Jeitler, ${ }^{2, b}$ N. Krammer, ${ }^{2}$ I. Krätschmer, ${ }^{2}$ D. Liko, ${ }^{2}$ T. Madlener, ${ }^{2}$ I. Mikulec, ${ }^{2}$ N. Rad, ${ }^{2}$ J. Schieck, ${ }^{2, b}$ R. Schöfbeck, ${ }^{2}$ M. Spanring, ${ }^{2}$ D. Spitzbart, ${ }^{2}$ W. Waltenberger, ${ }^{2}$ C.-E. Wulz, ${ }^{2, b}$ M. Zarucki, ${ }^{2}$ V. Drugakov, ${ }^{3}$ V. Mossolov, ${ }^{3}$ J. Suarez Gonzalez, ${ }^{3}$ M. R. Darwish, ${ }^{4}$ E. A. De Wolf, ${ }^{4}$ D. Di Croce, ${ }^{4}$ X. Janssen, ${ }^{4}$ A. Lelek, ${ }^{4}$ M. Pieters, ${ }^{4}$ H. Rejeb Sfar, ${ }^{4}$ H. Van Haevermaet, ${ }^{4}$ P. Van Mechelen, ${ }^{4}$ S. Van Putte, ${ }^{4}$ N. Van Remortel, ${ }^{4}$ F. Blekman, ${ }^{5}$ E. S. Bols, ${ }^{5}$ S. S. Chhibra, ${ }^{5}$ J. D'Hondt, ${ }^{5}$ J. De Clercq,${ }^{5}$ D. Lontkovskyi, ${ }^{5}$ S. Lowette, ${ }^{5}$ I. Marchesini, ${ }^{5}$ S. Moortgat, ${ }^{5}$ Q. Python, ${ }^{5}$ K. Skovpen, ${ }^{5}$ S. Tavernier, ${ }^{5}$ W. Van Doninck, ${ }^{5}$ P. Van Mulders, ${ }^{5}$ D. Beghin, ${ }^{6}$ B. Bilin, ${ }^{6}$ H. Brun, ${ }^{6}$ B. Clerbaux ${ }^{6}$ G. De Lentdecker, ${ }^{6}$ H. Delannoy, ${ }^{6}$ B. Dorney, ${ }^{6}$ L. Favart, ${ }^{6}$ A. Grebenyuk, ${ }^{6}$ A. K. Kalsi, ${ }^{6}$ A. Popov, ${ }^{6}$ N. Postiau, ${ }^{6}$ E. Starling, ${ }^{6}$ L. Thomas, ${ }^{6}$ C. Vander Velde, ${ }^{6}$ P. Vanlaer, ${ }^{6}$ D. Vannerom, ${ }^{6}$ T. Cornelis, ${ }^{7}$ D. Dobur, ${ }^{7}$ I. Khvastunov, ${ }^{7, \mathrm{c}}$ M. Niedziela, ${ }^{7}$ C. Roskas, ${ }^{7}$ M. Tytgat, ${ }^{7}$ W. Verbeke, ${ }^{7}$ B. Vermassen, ${ }^{7}$ M. Vit, ${ }^{7}$ O. Bondu, ${ }^{8}$ G. Bruno, ${ }^{8}$ C. Caputo, ${ }^{8}$ P. David, ${ }^{8}$ C. Delaere, ${ }^{8}$ M. Delcourt ${ }^{8}$ A. Giammanco, ${ }^{8}$ V. Lemaitre, ${ }^{8}$ J. Prisciandaro, ${ }^{8}$ A. Saggio, ${ }^{8}$ M. Vidal Marono, ${ }^{8}$ P. Vischia, ${ }^{8}$ J. Zobec ${ }^{8}$ F. L. Alves, ${ }^{9}$ G. A. Alves, ${ }^{9}$ G. Correia Silva, ${ }^{9}$ C. Hensel, ${ }^{9}$ A. Moraes, ${ }^{9}$ P. Rebello Teles, ${ }^{9}$ E. Belchior Batista Das Chagas, ${ }^{10}$ W. Carvalho, ${ }^{10}$ J. Chinellato, ${ }^{10, d}$ E. Coelho, ${ }^{10}$ E. M. Da Costa, ${ }^{10}$ G. G. Da Silveira, ${ }^{10, \mathrm{e}}$ D. De Jesus Damiao, ${ }^{10}$ C. De Oliveira Martins, ${ }^{10}$ S. Fonseca De Souza, ${ }^{10}$ L. M. Huertas Guativa, ${ }^{10}$ H. Malbouisson, ${ }^{10}$ J. Martins,${ }^{10, f}$ D. Matos Figueiredo, ${ }^{10}$ M. Medina Jaime,${ }^{10, g}$ M. Melo De Almeida, ${ }^{10}$ C. Mora Herrera,${ }^{10}$ L. Mundim, ${ }^{10}$ H. Nogima, ${ }^{10}$ W. L. Prado Da Silva, ${ }^{10}$ L. J. Sanchez Rosas, ${ }^{10}$ A. Santoro, ${ }^{10}$ A. Sznajder, ${ }^{10}$ M. Thiel, ${ }^{10}$

E. J. Tonelli Manganote, ${ }^{10, d}$ F. Torres Da Silva De Araujo, ${ }^{10}$ A. Vilela Pereira,${ }^{10}$ C. A. Bernardes, ${ }^{11 a}$ L. Calligaris, ${ }^{11 a}$ T. R. Fernandez Perez Tomei, ${ }^{11 \mathrm{a}}$ E. M. Gregores, ${ }^{11 \mathrm{a}, 1 \mathrm{~b}}$ D. S. Lemos, ${ }^{11 \mathrm{a}}$ P. G. Mercadante, ${ }^{11 \mathrm{a}, 1 \mathrm{~b}}{ }^{\mathrm{b}}$ S. F. Novaes, ${ }^{1 \mathrm{a}}$ Sandra S. Padula, ${ }^{11 a}$ A. Aleksandrov, ${ }^{12}$ G. Antchev, ${ }^{12}$ R. Hadjiiska, ${ }^{12}$ P. Iaydjiev, ${ }^{12}$ M. Misheva, ${ }^{12}$ M. Rodozov, ${ }^{12}$ M. Shopova, ${ }^{12}$ G. Sultanov, ${ }^{12}$ M. Bonchev, ${ }^{13}$ A. Dimitrov, ${ }^{13}$ T. Ivanov, ${ }^{13}$ L. Litov, ${ }^{13}$ B. Pavlov, ${ }^{13}$ P. Petkov, ${ }^{13}$ W. Fang, ${ }^{14, h}$ X. Gao, ${ }^{14, h}$ L. Yuan, ${ }^{14}$ G. M. Chen, ${ }^{16}$ H. S. Chen, ${ }^{16}$ M. Chen, ${ }^{16}$ C. H. Jiang, ${ }^{16}$ D. Leggat,${ }^{16}$ H. Liao, ${ }^{16}$ Z. Liu, ${ }^{16}$ A. Spiezia, ${ }^{16}$ 
J. Tao, ${ }^{16}$ E. Yazgan, ${ }^{16}$ H. Zhang, ${ }^{16}$ S. Zhang, ${ }^{16, i}$ J. Zhao, ${ }^{16}$ A. Agapitos, ${ }^{17}$ Y. Ban, ${ }^{17}$ G. Chen, ${ }^{17}$ A. Levin, ${ }^{17}$ J. Li, ${ }^{17}$ L. Li, ${ }^{17}$ Q. Li ${ }^{17}$ Y. Mao, ${ }^{17}$ S. J. Qian, ${ }^{17}$ D. Wang, ${ }^{17}$ Q. Wang, ${ }^{17}$ M. Ahmad, ${ }^{15}$ Z. Hu, ${ }^{15}$ Y. Wang, ${ }^{15}$ M. Xiao, ${ }^{18}$ C. Avila, ${ }^{19}$ A. Cabrera, ${ }^{19}$ C. Florez, ${ }^{19}$ C. F. González Hernández, ${ }^{19}$ M. A. Segura Delgado, ${ }^{19}$ J. Mejia Guisao, ${ }^{20}$ J. D. Ruiz Alvarez ${ }^{20}$ C. A. Salazar González, ${ }^{20}$ N. Vanegas Arbelaez, ${ }^{20}$ D. Giljanović, ${ }^{21}$ N. Godinovic, ${ }^{21}$ D. Lelas, ${ }^{21}$ I. Puljak, ${ }^{21}$ T. Sculac, ${ }^{21}$ Z. Antunovic, ${ }^{22}$ M. Kovac, ${ }^{22}$ V. Brigljevic, ${ }^{23}$ D. Ferencek, ${ }^{23}$ K. Kadija, ${ }^{23}$ B. Mesic, ${ }^{23}$ M. Roguljic, ${ }^{23}$ A. Starodumov, ${ }^{23, j}$ T. Susa, ${ }^{23}$ M. W. Ather, ${ }^{24}$ A. Attikis, ${ }^{24}$ E. Erodotou, ${ }^{24}$ A. Ioannou, ${ }^{24}$ M. Kolosova, ${ }^{24}$ S. Konstantinou, ${ }^{24}$

G. Mavromanolakis, ${ }^{24}$ J. Mousa, ${ }^{24}$ C. Nicolaou, ${ }^{24}$ F. Ptochos,${ }^{24}$ P. A. Razis, ${ }^{24}$ H. Rykaczewski, ${ }^{24}$ D. Tsiakkouri, ${ }^{24}$ M. Finger ${ }^{25, \mathrm{k}}$ M. Finger Jr., ${ }^{25, \mathrm{k}}$ A. Kveton, ${ }^{25} \mathrm{~J}$. Tomsa, ${ }^{25}$ E. Ayala, ${ }^{26}$ E. Carrera Jarrin, ${ }^{27}$ S. Elgammal, ${ }^{28,1}$ E. Salama, ${ }^{28, \mathrm{~m}, 1}$ S. Bhowmik, ${ }^{29}$ A. Carvalho Antunes De Oliveira, ${ }^{29}$ R. K. Dewanjee, ${ }^{29}$ K. Ehataht, ${ }^{29}$ M. Kadastik,${ }^{29}$ M. Raidal, ${ }^{29}$ C. Veelken, ${ }^{29}$ P. Eerola,${ }^{30}$ L. Forthomme, ${ }^{30}$ H. Kirschenmann,${ }^{30}$ K. Osterberg, ${ }^{30}$ M. Voutilainen,${ }^{30}$ F. Garcia, ${ }^{31}$

J. Havukainen, ${ }^{31}$ J. K. Heikkilä, ${ }^{31}$ V. Karimäki, ${ }^{31}$ M. S. Kim, ${ }^{31}$ R. Kinnunen, ${ }^{31}$ T. Lampén, ${ }^{31}$ K. Lassila-Perini, ${ }^{31}$ S. Laurila, ${ }^{31}$ S. Lehti, ${ }^{31}$ T. Lindén, ${ }^{31}$ P. Luukka, ${ }^{31}$ T. Mäenpää, ${ }^{31}$ H. Siikonen, ${ }^{31}$ E. Tuominen, ${ }^{31}$ J. Tuominiemi, ${ }^{31}$ T. Tuuva, ${ }^{32}$ M. Besancon, ${ }^{33}$ F. Couderc, ${ }^{33}$ M. Dejardin, ${ }^{33}$ D. Denegri, ${ }^{33}$ B. Fabbro, ${ }^{33}$ J. L. Faure, ${ }^{33}$ F. Ferri, ${ }^{33}$ S. Ganjour, ${ }^{33}$ A. Givernaud, ${ }^{33}$ P. Gras, ${ }^{33}$ G. Hamel de Monchenault, ${ }^{33}$ P. Jarry, ${ }^{33}$ C. Leloup, ${ }^{33}$ B. Lenzi, ${ }^{33}$ E. Locci, ${ }^{33}$ J. Malcles, ${ }^{33}$ J. Rander, ${ }^{33}$ A. Rosowsky, ${ }^{33}$ M. Ö. Sahin, ${ }^{33}$ A. Savoy-Navarro, ${ }^{33, n}$ M. Titov, ${ }^{33}$ G. B. Yu, ${ }^{33}$ S. Ahuja, ${ }^{34}$ C. Amendola, ${ }^{34}$ F. Beaudette, ${ }^{34}$ P. Busson, ${ }^{34}$ C. Charlot,${ }^{34}$ B. Diab,${ }^{34}$ G. Falmagne, ${ }^{34}$ R. Granier de Cassagnac, ${ }^{34}$ I. Kucher, ${ }^{34}$ A. Lobanov, ${ }^{34}$ C. Martin Perez, ${ }^{34}$ M. Nguyen, ${ }^{34}$ C. Ochando, ${ }^{34}$ P. Paganini, ${ }^{34}$ J. Rembser, ${ }^{34}$ R. Salerno, ${ }^{34}$ J. B. Sauvan, ${ }^{34}$ Y. Sirois,${ }^{34}$ A. Zabi, ${ }^{34}$ A. Zghiche, ${ }^{34}$ J.-L. Agram, ${ }^{35,0}$ J. Andrea, ${ }^{35}$ D. Bloch, ${ }^{35}$ G. Bourgatte, ${ }^{35}$ J.-M. Brom, ${ }^{35}$ E. C. Chabert, ${ }^{35}$ C. Collard, ${ }^{35}$ E. Conte, ${ }^{35,0}$ J.-C. Fontaine ${ }^{35,0}$ D. Gelé, ${ }^{35}$ U. Goerlach,${ }^{35}$ M. Jansová, ${ }^{35}$ A.-C. Le Bihan,${ }^{35}$ N. Tonon, ${ }^{35}$ P. Van Hove,${ }^{35}$ S. Gadrat,${ }^{36}$ S. Beauceron, ${ }^{37}$ C. Bernet, ${ }^{37}$ G. Boudoul, ${ }^{37}$ C. Camen,${ }^{37}$ A. Carle, ${ }^{37}$ N. Chanon, ${ }^{37}$ R. Chierici, ${ }^{37}$ D. Contardo,${ }^{37}$ P. Depasse, ${ }^{37}$ H. El Mamouni, ${ }^{37}$ J. Fay, ${ }^{37}$ S. Gascon, ${ }^{37}$ M. Gouzevitch, ${ }^{37}$ B. Ille, ${ }^{37}$ Sa. Jain, ${ }^{37}$ F. Lagarde,${ }^{37}$ I. B. Laktineh, ${ }^{37}$ H. Lattaud,${ }^{37}$ A. Lesauvage, ${ }^{37}$ M. Lethuillier, ${ }^{37}$ L. Mirabito, ${ }^{37}$ S. Perries,${ }^{37}$ V. Sordini,${ }^{37}$ L. Torterotot,${ }^{37}$

G. Touquet, ${ }^{37}$ M. Vander Donckt, ${ }^{37}$ S. Viret ${ }^{37}$ T. Toriashvili, ${ }^{38, p}$ Z. Tsamalaidze, ${ }^{39, k}$ C. Autermann,${ }^{40}$ L. Feld, ${ }^{40}$ M. K. Kiesel, ${ }^{40}$ K. Klein, ${ }^{40}$ M. Lipinski, ${ }^{40}$ D. Meuser, ${ }^{40}$ A. Pauls, ${ }^{40}$ M. Preuten, ${ }^{40}$ M. P. Rauch, ${ }^{40}$ J. Schulz, ${ }^{40}$ M. Teroerde,${ }^{40}$ B. Wittmer, ${ }^{40}$ M. Erdmann, ${ }^{41}$ B. Fischer, ${ }^{41}$ S. Ghosh,${ }^{41}$ T. Hebbeker, ${ }^{41}$ K. Hoepfner, ${ }^{41}$ H. Keller, ${ }^{41}$ L. Mastrolorenzo, ${ }^{41}$ M. Merschmeyer, ${ }^{41}$ A. Meyer, ${ }^{41}$ P. Millet, ${ }^{41}$ G. Mocellin, ${ }^{41}$ S. Mondal, ${ }^{41}$ S. Mukherjee, ${ }^{41}$ D. Noll, ${ }^{41}$ A. Novak, ${ }^{41}$ T. Pook, ${ }^{41}$ A. Pozdnyakov, ${ }^{41}$ T. Quast, ${ }^{41}$ M. Radziej, ${ }^{41}$ Y. Rath, ${ }^{41}$ H. Reithler, ${ }^{41}$ J. Roemer, ${ }^{41}$ A. Schmidt, ${ }^{41}$ S. C. Schuler, ${ }^{41}$

A. Sharma, ${ }^{41}$ S. Wiedenbeck, ${ }^{41}$ S. Zaleski, ${ }^{41}$ G. Flügge, ${ }^{42}$ W. Haj Ahmad ${ }^{42, q}$ O. Hlushchenko, ${ }^{42}$ T. Kress, ${ }^{42}$ T. Müller ${ }^{42}$ A. Nowack,${ }^{42}$ C. Pistone,${ }^{42}$ O. Pooth,${ }^{42}$ D. Roy, ${ }^{42}$ H. Sert, ${ }^{42}$ A. Stahl,${ }^{42, r}$ M. Aldaya Martin, ${ }^{43}$ P. Asmuss, ${ }^{43}$ I. Babounikau, ${ }^{43}$ H. Bakhshiansohi, ${ }^{43}$ K. Beernaert, ${ }^{43}$ O. Behnke, ${ }^{43}$ A. Bermúdez Martínez, ${ }^{43}$ D. Bertsche, ${ }^{43}$ A. A. Bin Anuar, ${ }^{43}$ K. Borras, ${ }^{43, s}$ V. Botta, ${ }^{43}$ A. Campbell, ${ }^{43}$ A. Cardini,${ }^{43}$ P. Connor, ${ }^{43}$ S. Consuegra Rodríguez, ${ }^{43}$ C. Contreras-Campana, ${ }^{43}$ V. Danilov, ${ }^{43}$ A. De Wit, ${ }^{43}$ M. M. Defranchis, ${ }^{43}$ C. Diez Pardos ${ }^{43}$ D. Domínguez Damiani, ${ }^{43}$ G. Eckerlin, ${ }^{43}$ D. Eckstein, ${ }^{43}$ T. Eichhorn, ${ }^{43}$ A. Elwood, ${ }^{43}$ E. Eren, ${ }^{43}$ E. Gallo, ${ }^{43, t}$ A. Geiser, ${ }^{43}$ A. Grohsjean, ${ }^{43}$ M. Guthoff, ${ }^{43}$ M. Haranko, ${ }^{43}$ A. Harb,${ }^{43}$ A. Jafari, ${ }^{43}$ N. Z. Jomhari, ${ }^{43}$ H. Jung, ${ }^{43}$ A. Kasem, ${ }^{43,8}$ M. Kasemann, ${ }^{43}$ H. Kaveh,${ }^{43}$ J. Keaveney, ${ }^{43}$ C. Kleinwort, ${ }^{43}$ J. Knolle, ${ }^{43}$ D. Krücker, ${ }^{43}$ W. Lange, ${ }^{43}$ T. Lenz, ${ }^{43}$ J. Lidrych,${ }^{43}$ K. Lipka, ${ }^{43}$ W. Lohmann, ${ }^{43, u}$ R. Mankel, ${ }^{43}$ I.-A. Melzer-Pellmann, ${ }^{43}$ A. B. Meyer, ${ }^{43}$ M. Meyer, ${ }^{43}$ M. Missiroli, ${ }^{43}$ G. Mittag, ${ }^{43}$ J. Mnich, ${ }^{43}$ A. Mussgiller, ${ }^{43}$ V. Myronenko, ${ }^{43}$ D. Pérez Adán, ${ }^{43}$ S. K. Pflitsch, ${ }^{43}$ D. Pitzl, ${ }^{43}$ A. Raspereza, ${ }^{43}$ A. Saibel,${ }^{43}$ M. Savitskyi, ${ }^{43}$ V. Scheurer, ${ }^{43}$ P. Schütze ${ }^{43}$ C. Schwanenberger ${ }^{43}$ R. Shevchenko, ${ }^{43}$ A. Singh, ${ }^{43}$ H. Tholen, ${ }^{43}$ O. Turkot ${ }^{43}$ A. Vagnerini,${ }^{43}$ M. Van De Klundert,${ }^{43}$ R. Walsh, ${ }^{43}$ Y. Wen, ${ }^{43}$ K. Wichmann, ${ }^{43}$ C. Wissing, ${ }^{43}$ O. Zenaiev, ${ }^{43}$ R. Zlebcik, ${ }^{43}$ R. Aggleton, ${ }^{44}$ S. Bein, ${ }^{44}$ L. Benato, ${ }^{44}$ A. Benecke, ${ }^{44}$ V. Blobel, ${ }^{44}$ T. Dreyer, ${ }^{44}$ A. Ebrahimi, ${ }^{44}$ F. Feindt, ${ }^{44}$ A. Fröhlich ${ }^{44}$ C. Garbers, ${ }^{44}$ E. Garutti, ${ }^{44}$ D. Gonzalez, ${ }^{44}$ P. Gunnellini, ${ }^{44}$ J. Haller,${ }^{44}$ A. Hinzmann, ${ }^{44}$ A. Karavdina, ${ }^{44}$ G. Kasieczka, ${ }^{44}$ R. Klanner, ${ }^{44}$ R. Kogler ${ }^{44}$ N. Kovalchuk, ${ }^{44}$ S. Kurz, ${ }^{44}$ V. Kutzner, ${ }^{44}$ J. Lange, ${ }^{44}$ T. Lange, ${ }^{44}$ A. Malara,${ }^{44}$ J. Multhaup, ${ }^{44}$ C. E. N. Niemeyer ${ }^{44}$ A. Perieanu, ${ }^{44}$ A. Reimers ${ }^{44}$ O. Rieger ${ }^{44}$ C. Scharf ${ }^{44}$ P. Schleper, ${ }^{44}$ S. Schumann, ${ }^{44}$ J. Schwandt, ${ }^{44}$ J. Sonneveld, ${ }^{44}$ H. Stadie, ${ }^{44}$ G. Steinbrück, ${ }^{44}$ F. M. Stober, ${ }^{44}$ B. Vormwald, ${ }^{44}$ I. Zoi ${ }^{44}$ M. Akbiyik, ${ }^{45}$ C. Barth ${ }^{45}$ M. Baselga, ${ }^{45}$ S. Baur, ${ }^{45}$ T. Berger, ${ }^{45}$ E. Butz, ${ }^{45}$ R. Caspart, ${ }^{45}$ T. Chwalek, ${ }^{45}$ W. De Boer, ${ }^{45}$ A. Dierlamm, ${ }^{45}$ K. El Morabit, ${ }^{45}$ N. Faltermann, ${ }^{45}$ M. Giffels, ${ }^{45}$ P. Goldenzweig, ${ }^{45}$ A. Gottmann, ${ }^{45}$ M. A. Harrendorf, ${ }^{45}$ F. Hartmann, ${ }^{45, \mathrm{r}}$ U. Husemann, ${ }^{45}$ S. Kudella, ${ }^{45}$ S. Mitra ${ }^{45}$ M. U. Mozer, ${ }^{45}$ D. Müller ${ }^{45}$ Th. Müller, ${ }^{45}$ M. Musich, ${ }^{45}$ A. Nürnberg, ${ }^{45}$ G. Quast, ${ }^{45}$ K. Rabbertz ${ }^{45}$ M. Schröder, ${ }^{45}$ I. Shvetsov, ${ }^{45}$ H. J. Simonis, ${ }^{45}$ R. Ulrich, ${ }^{45}$ M. Wassmer, ${ }^{45}$ M. Weber ${ }^{45}$ C. Wöhrmann, ${ }^{45}$ R. Wolf, ${ }^{45}$ G. Anagnostou, ${ }^{46}$ P. Asenov, ${ }^{46}$ G. Daskalakis ${ }^{46}$ T. Geralis, ${ }^{46}$ A. Kyriakis, ${ }^{46}$ D. Loukas, ${ }^{46}$ G. Paspalaki, ${ }^{46}$ M. Diamantopoulou, ${ }^{47}$ G. Karathanasis, ${ }^{47}$ P. Kontaxakis, ${ }^{47}$ 
A. Manousakis-katsikakis, ${ }^{47}$ A. Panagiotou ${ }^{47}$ I. Papavergou,${ }^{47}$ N. Saoulidou, ${ }^{47}$ A. Stakia, ${ }^{47}$ K. Theofilatos, ${ }^{47}$ K. Vellidis,${ }^{47}$ E. Vourliotis ${ }^{47}$ G. Bakas, ${ }^{48}$ K. Kousouris,${ }^{48}$ I. Papakrivopoulos, ${ }^{48}$ G. Tsipolitis, ${ }^{48}$ I. Evangelou, ${ }^{49}$ C. Foudas, ${ }^{49}$ P. Gianneios, ${ }^{49}$ P. Katsoulis, ${ }^{49}$ P. Kokkas, ${ }^{49}$ S. Mallios, ${ }^{49}$ K. Manitara, ${ }^{49}$ N. Manthos, ${ }^{49}$ I. Papadopoulos, ${ }^{49}$ J. Strologas,${ }^{49}$ F. A. Triantis, ${ }^{49}$ D. Tsitsonis, ${ }^{49}$ M. Bartók ${ }^{50, v}$ R. Chudasama,${ }^{50}$ M. Csanad ${ }^{50}$ P. Major ${ }^{50}$ K. Mandal, ${ }^{50}$ A. Mehta, ${ }^{50}$ M. I. Nagy, ${ }^{50}$ G. Pasztor,${ }^{50}$ O. Surányi,${ }^{50}$ G. I. Veres,${ }^{50}$ G. Bencze, ${ }^{51}$ C. Hajdu ${ }^{51}$ D. Horvath, ${ }^{51, w}$ F. Sikler, ${ }^{51}$ T. Á. Vámi ${ }^{51}$ V. Veszpremi ${ }^{51}$ G. Vesztergombi, ${ }^{51, \mathrm{a}, \mathrm{x}}$ N. Beni ${ }^{52}$ S. Czellar, ${ }^{52}$ J. Karancsi, ${ }^{52, \mathrm{v}}$ A. Makovec, ${ }^{52}$ J. Molnar, ${ }^{52}$ Z. Szillasi, ${ }^{52}$ P. Raics, ${ }^{53}$ D. Teyssier, ${ }^{53}$ Z. L. Trocsanyi,${ }^{53}$ B. Ujvari, ${ }^{53}$ T. Csorgo,${ }^{54}$ W. J. Metzger, ${ }^{54}$ F. Nemes,${ }^{54}$ T. Novak, ${ }^{54}$ S. Choudhury, ${ }^{55}$ J. R. Komaragiri, ${ }^{55}$ P. C. Tiwari, ${ }^{55}$ S. Bahinipati, ${ }^{56, y}$ C. Kar, ${ }^{56}$ G. Kole, ${ }^{56}$ P. Mal, ${ }^{56}$

V. K. Muraleedharan Nair Bindhu, ${ }^{56}$ A. Nayak, ${ }^{56, z}$ D. K. Sahoo, ${ }^{56, y}$ S. K. Swain, ${ }^{56}$ S. Bansal,${ }^{57}$ S. B. Beri, ${ }^{57}$ V. Bhatnagar, ${ }^{57}$ S. Chauhan, ${ }^{57}$ R. Chawla, ${ }^{57}$ N. Dhingra, ${ }^{57}$ R. Gupta, ${ }^{57}$ A. Kaur,${ }^{57}$ M. Kaur, ${ }^{57}$ S. Kaur, ${ }^{57}$ P. Kumari, ${ }^{57}$ M. Lohan, ${ }^{57}$ M. Meena, ${ }^{57}$ K. Sandeep ${ }^{57}$ S. Sharma, ${ }^{57}$ J. B. Singh, ${ }^{57}$ A. K. Virdi, ${ }^{57}$ A. Bhardwaj, ${ }^{58}$ B. C. Choudhary,${ }^{58}$ R. B. Garg,${ }^{58}$ M. Gola ${ }^{58}$ S. Keshri, ${ }^{58}$ Ashok Kumar,${ }^{58}$ M. Naimuddin,${ }^{58}$ P. Priyanka ${ }^{58}$ K. Ranjan, ${ }^{58}$ Aashaq Shah,${ }^{58}$ R. Sharma, ${ }^{58}$ R. Bhardwaj, ${ }^{59, \text { aa }}$ M. Bharti, ${ }^{59, \text { aa }}$ R. Bhattacharya, ${ }^{59}$ S. Bhattacharya ${ }^{59}$ U. Bhawandeep, ${ }^{59, \text { aa }}$ D. Bhowmik, ${ }^{59}$ S. Dutta, ${ }^{59}$ S. Ghosh, ${ }^{59}$ B. Gomber, ${ }^{59, b b}$ M. Maity,${ }^{59, c c}$ K. Mondal, ${ }^{59}$ S. Nandan, ${ }^{59}$ A. Purohit, ${ }^{59}$ P. K. Rout,${ }^{59}$ G. Saha, ${ }^{59}$ S. Sarkar,${ }^{59}$ T. Sarkar, ${ }^{59, c c}$ M. Sharan, ${ }^{59}$ B. Singh, ${ }^{59, \text { aa }}$ S. Thakur, ${ }^{59, \text { aa }}$ P. K. Behera, ${ }^{60}$ P. Kalbhor, ${ }^{60}$ A. Muhammad, ${ }^{60}$ P. R. Pujahari, ${ }^{60}$ A. Sharma, ${ }^{60}$ A. K. Sikdar, ${ }^{60}$ D. Dutta, ${ }^{61}$ V. Jha,${ }^{61}$ V. Kumar, ${ }^{61}$ D. K. Mishra, ${ }^{61}$ P. K. Netrakanti, ${ }^{61}$ L. M. Pant, ${ }^{61}$ P. Shukla, ${ }^{61}$ T. Aziz, ${ }^{62}$ M. A. Bhat, ${ }^{62}$ S. Dugad, ${ }^{62}$ G. B. Mohanty, ${ }^{62}$ N. Sur, ${ }^{62}$ Ravindra Kumar Verma, ${ }^{62}$ S. Banerjee, ${ }^{63}$ S. Bhattacharyaa ${ }^{63}$ S. Chatterjee, ${ }^{63}$ P. Das, ${ }^{63}$ M. Guchait, ${ }^{63}$ S. Karmakar, ${ }^{63}$ S. Kumar, ${ }^{63}$ G. Majumder, ${ }^{63}$ K. Mazumdar,${ }^{63}$ N. Sahoo, ${ }^{63}$ S. Sawant, ${ }^{63}$ S. Dube, ${ }^{64}$ B. Kansal,${ }^{64}$ A. Kapoor, ${ }^{64}$ K. Kothekar,${ }^{64}$ S. Pandey,${ }^{64}$ A. Rane,${ }^{64}$ A. Rastogi,${ }^{64}$ S. Sharma, ${ }^{64}$ S. Chenarani, ${ }^{65, \text { dd }}$ E. Eskandari Tadavani, ${ }^{65}$ S. M. Etesami,${ }^{65, d d}$ M. Khakzad, ${ }^{65}$ M. Mohammadi Najafabadi, ${ }^{65}$ M. Naseri, ${ }^{65}$

F. Rezaei Hosseinabadi, ${ }^{65}$ M. Felcini, ${ }^{66}$ M. Grunewald ${ }^{66}$ M. Abbrescia, ${ }^{67 a, 67 b}$ R. Aly, ${ }^{67 a, 67 b, e e}$ C. Calabria, ${ }^{67 a, 67 b}$

A. Colaleo, ${ }^{67 a}$ D. Creanza, ${ }^{67 a, 67 c}$ L. Cristella, ${ }^{67 a, 67 b}$ N. De Filippis, ${ }^{67,67 c}$ M. De Palma, ${ }^{67,67 b}$ A. Di Florio, ${ }^{67 a, 67 b}$ W. Elmetenawee, ${ }^{67 a, 67 b}$ L. Fiore, ${ }^{67 a}$ A. Gelmi, ${ }^{67 a, 67 b}$ G. Iaselli, ${ }^{67 a, 67 c}$ M. Ince, ${ }^{67 a, 67 b}$ S. Lezki, ${ }^{67 a, 67 b}$ G. Maggi, ${ }^{67 a, 67 c}$ M. Maggi, ${ }^{67 a}$ J. A. Merlin, ${ }^{67 a}$ G. Miniello, ${ }^{67,67 b}$ S. My, ${ }^{67 a, 67 b}$ S. Nuzzo, ${ }^{67 a, 67 b}$ A. Pompili, ${ }^{67 a, 67 b}$ G. Pugliese, ${ }^{67 a, 67 \mathrm{c}}$ R. Radogna, ${ }^{67 a}$ A. Ranieri, ${ }^{67 a}$ G. Selvaggi, ${ }^{67 a, 67 b}$ L. Silvestris, ${ }^{67 a}$ F. M. Simone,${ }^{67,67 b}$ R. Venditti, ${ }^{67 a}$ P. Verwilligen, ${ }^{67 a}$ G. Abbiendi, ${ }^{68 \mathrm{a}}$ C. Battilana, ${ }^{68 a, 68 b}$ D. Bonacorsi, ${ }^{68 a, 68 b}$ L. Borgonovi, ${ }^{68 a, 68 b}$ S. Braibant-Giacomelli, ${ }^{68,68 b}$

R. Campanini, ${ }^{68 a, 68 b}$ P. Capiluppi, ${ }^{68 a, 68 b}$ A. Castro, ${ }^{68 a, 68 b}$ F. R. Cavallo, ${ }^{68 a}$ C. Ciocca, ${ }^{68 a}$ G. Codispoti ${ }^{68 a, 68 b}$ M. Cuffiani ${ }^{68 a, 68 b}$ G. M. Dallavalle, ${ }^{68 a}$ F. Fabbri, ${ }^{68 a}$ A. Fanfani, ${ }^{68 a, 68 b}$ E. Fontanesi ${ }^{68 a, 68 b}$ P. Giacomelli, ${ }^{68 a}$ C. Grandi, ${ }^{68 a}$ L. Guiducci, ${ }^{68 a, 68 b}$ F. Iemmi, ${ }^{68 a, 68 b}$ S. Lo Meo, ${ }^{68 a, f f}$ S. Marcellini ${ }^{68 \mathrm{a}}$ G. Masetti, ${ }^{68 \mathrm{a}}$ F. L. Navarria, ${ }^{68 \mathrm{a}, 68 \mathrm{~b}}$ A. Perrotta, ${ }^{68 \mathrm{a}}$ F. Primavera, ${ }^{68 \mathrm{a}, 68 \mathrm{~b}}$ A. M. Rossi, ${ }^{68 a, 68 b}$ T. Rovelli, ${ }^{68 a, 68 b}$ G. P. Siroli, ${ }^{68 a, 68 b}$ N. Tosi, ${ }^{68 a}$ S. Albergo,${ }^{69 a, 69 b, g g}$ S. Costa, ${ }^{69 a, 69 b}$ A. Di Mattia, ${ }^{69 a}$ R. Potenza, ${ }^{69 a, 69 b}$ A. Tricomi, ${ }^{69 a, 69 b, g g}$ C. Tuve,${ }^{69,69 b}$ G. Barbagli, ${ }^{70 a}$ A. Cassese, ${ }^{70 a}$ R. Ceccarelli, ${ }^{70 a}$ V. Ciulli, ${ }^{70 a, 70 b}$ C. Civinini, ${ }^{70 \mathrm{a}}$ R. D’Alessandro, ${ }^{70 a, 70 \mathrm{~b}}$ F. Fiori, ${ }^{70 \mathrm{a}, 70 \mathrm{~b}}$ E. Focardi, ${ }^{70 \mathrm{a}, 70 \mathrm{~b}}$ G. Latino, ${ }^{70 a, 70 \mathrm{~b}}$ P. Lenzi,${ }^{70 a, 70 \mathrm{~b}}$ M. Meschini, ${ }^{70 \mathrm{a}}$ S. Paoletti, ${ }^{70 a}$ G. Sguazzoni, ${ }^{70 a}$ L. Viliani, ${ }^{70 a}$ L. Benussi, ${ }^{71}$ S. Bianco,${ }^{71}$ D. Piccolo, ${ }^{71}$ M. Bozzo, ${ }^{72 a, 72 b}$ F. Ferro, ${ }^{72 a}$ R. Mulargia, ${ }^{72 a, 72 b}$ E. Robutti, ${ }^{72 a}$ S. Tosi, ${ }^{72 a, 72 b}$ A. Benaglia, ${ }^{73 a}$ A. Beschi, ${ }^{73 a, 73 b}$ F. Brivio, ${ }^{73 a, 73 b}$ V. Ciriolo, ${ }^{73 a, 73 b, r}$ S. Di Guida, ${ }^{73 a, 73 b, r}$ M. E. Dinardo, ${ }^{73 a, 73 b}$ P. Dini, ${ }^{73 a}$ S. Gennai, ${ }^{73 a}$ A. Ghezzi ${ }^{73 a, 73 b}$ P. Govoni, ${ }^{73 a, 73 b}$ L. Guzzi, ${ }^{73 a, 73 b}$ M. Malberti, ${ }^{73 a}$ S. Malvezzi, ${ }^{73 a}$ D. Menasce, ${ }^{73 a}$ F. Monti, ${ }^{73 a, 73 b}$ L. Moroni, ${ }^{73 a}$ M. Paganoni ${ }^{73 a, 73 b}$ D. Pedrini, ${ }^{73 a}$ S. Ragazzi, ${ }^{73 a, 73 b}$ T. Tabarelli de Fatis, ${ }^{73 a, 73 b}$ D. Zuolo, ${ }^{73 a, 73 b}$ S. Buontempo, ${ }^{74 a}$ N. Cavallo, ${ }^{74 a, 74 c}$ A. De Iorio, ${ }^{74 a, 74 b}$ A. Di Crescenzo ${ }^{74 a, 74 b}$ F. Fabozzi, ${ }^{74 a, 74 c}$ F. Fienga, ${ }^{74 a}$ G. Galati, ${ }^{74 a}$ A. O. M. Iorio, ${ }^{74 a, 74 b}$ L. Lista ${ }^{74 a, 74 b}$ S. Meola, ${ }^{74 a, 74 d, r}$

P. Paolucci, ${ }^{74 a, r}$ B. Rossi ${ }^{74 a}$ C. Sciacca $,{ }^{74 a}, 74 b$ E. Voevodina,${ }^{74 a, 74 b}$ P. Azzi,${ }^{75 a}$ N. Bacchetta, ${ }^{75 a}$ D. Bisello, ${ }^{75 a, 75 b}$ A. Boletti, ${ }^{75 a, 75 b}$ A. Bragagnolo, ${ }^{75 a, 75 b}$ R. Carlin, ${ }^{75 a, 75 b}$ P. Checchia, ${ }^{75 a}$ P. De Castro Manzano, ${ }^{75 a}$ T. Dorigo, ${ }^{75 a}$ U. Dosselli, ${ }^{75 a}$

F. Gasparini, ${ }^{75 a, 75 b}$ U. Gasparini, ${ }^{75 a, 75 b}$ A. Gozzelino, ${ }^{75 a}$ S. Y. Hoh, ${ }^{75 a, 75 b}$ P. Lujan, ${ }^{75 a}$ M. Margoni, ${ }^{75 a, 75 b}$

A. T. Meneguzzo, ${ }^{75 a, 75 b}$ J. Pazzini, ${ }^{75 a, 75 b}$ M. Presilla, ${ }^{75 a, 75 b}$ P. Ronchese, ${ }^{75 a, 75 b}$ R. Rossin, ${ }^{75 a, 75 b}$ F. Simonetto, ${ }^{75 a, 75 b}$ A. Tiko, ${ }^{75 a}$ M. Tosi, ${ }^{75 a, 75 b}$ M. Zanetti, ${ }^{75 a, 75 b}$ P. Zotto, ${ }^{75 a, 75 b}$ G. Zumerle, ${ }^{75 a, 75 b}$ A. Braghieri, ${ }^{76 a}$ D. Fiorina, ${ }^{76 a, 76 b}$ P. Montagna, ${ }^{76 a, 76 b}$ S. P. Ratti, ${ }^{76 a, 76 b}$ V. Re, ${ }^{76 a}$ M. Ressegotti, ${ }^{76 a, 76 b}$ C. Riccardi, ${ }^{76 a, 76 b}$ P. Salvini, ${ }^{76 a}$ I. Vai, ${ }^{76 a}$ P. Vitulo, ${ }^{76 a, 76 b}$ M. Biasini, ${ }^{77 a, 77 b}$ G. M. Bilei, ${ }^{77 a}$ D. Ciangottini, ${ }^{77 a, 77 b}$ L. Fanò, ${ }^{77 a, 77 b}$ P. Lariccia, ${ }^{77 a, 77 b}$ R. Leonardi, ${ }^{77 a, 77 b}$ E. Manoni, ${ }^{77 a}$ G. Mantovani, ${ }^{77 a, 77 b}$

V. Mariani, ${ }^{77 a, 77 b}$ M. Menichelli, ${ }^{77 a}$ A. Rossi, ${ }^{77 a, 77 b}$ A. Santocchia, ${ }^{77 a, 77 b}$ D. Spiga ${ }^{77 a}$ K. Androsov, ${ }^{78 a}$ P. Azzurri, ${ }^{78 a}$

G. Bagliesi, ${ }^{78 a}$ V. Bertacchi, ${ }^{78 a, 78 c}$ L. Bianchini, ${ }^{78 a}$ T. Boccali, ${ }^{78 a}$ R. Castaldi, ${ }^{78 a}$ M. A. Ciocci, ${ }^{78 a, 78 b}$ R. Dell'Orso, ${ }^{78 a}$

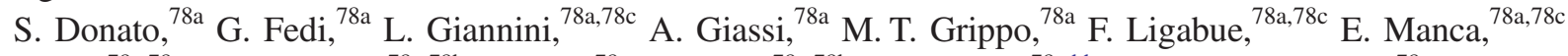

G. Mandorli, ${ }^{78 a, 78 c}$ A. Messineo, ${ }^{78 a, 78 b}$ F. Palla, ${ }^{78 a}$ A. Rizzi, ${ }^{78 a, 78 b}$ G. Rolandi, ${ }^{78 a, h h}$ S. Roy Chowdhury, ${ }^{78 a}$ A. Scribano, ${ }^{78 a}$ 
P. Spagnolo, ${ }^{78 a}$ R. Tenchini, ${ }^{78 a}$ G. Tonelli, ${ }^{78 a, 78 b}$ N. Turini, ${ }^{78 a}$ A. Venturi, ${ }^{78 a}$ P. G. Verdini, ${ }^{78 a}$ F. Cavallari, ${ }^{79 a}$ M. Cipriani,${ }^{79 a, 79 b}$ D. Del Re ${ }^{79 a, 79 b}$ E. Di Marco, ${ }^{79 a}$ M. Diemoz ${ }^{79 a}$ E. Longo, ${ }^{79 a, 79 b}$ P. Meridiani, ${ }^{79 a}$ G. Organtini, ${ }^{79 a, 79 b}$ F. Pandolfi, ${ }^{79 a}$ R. Paramatti, ${ }^{7 a, 79 b}$ C. Quaranta, ${ }^{79 a, 79 b}$ S. Rahatlou, ${ }^{79 a, 79 b}$ C. Rovelli, ${ }^{79 a}$ F. Santanastasio, ${ }^{79 a, 79 b}$ L. Soffi, ${ }^{79 a, 79 b}$ N. Amapane, ${ }^{80 \mathrm{a}, 80 \mathrm{~b}}$ R. Arcidiacono ${ }^{80 \mathrm{a}, 80 \mathrm{c}} \mathrm{S}$. Argiro, ${ }^{80 \mathrm{a}, 80 \mathrm{~b}}$ M. Arneodo, ${ }^{80 \mathrm{a}, 80 \mathrm{c}}$ N. Bartosik, ${ }^{80 \mathrm{a}}$ R. Bellan, ${ }^{80 \mathrm{a}, 80 \mathrm{~b}}$ A. Bellora, ${ }^{80 \mathrm{a}}$ C. Biino ${ }^{80 a}$ A. Cappati, ${ }^{80 a, 80 b}$ N. Cartiglia, ${ }^{80 a}$ S. Cometti, ${ }^{80 a}$ M. Costa,${ }^{80 a, 80 b}$ R. Covarelli, ${ }^{80,80 b}$ N. Demaria ${ }^{80 a}$ B. Kiani, ${ }^{80 a, 80 b}$ F. Legger, ${ }^{80 \mathrm{a}}$ C. Mariotti ${ }^{80 \mathrm{a}}$ S. Maselli, ${ }^{80 \mathrm{a}}$ E. Migliore ${ }^{80 \mathrm{a}, 80 \mathrm{~b}}$ V. Monaco, ${ }^{80 \mathrm{a}, 80 \mathrm{~b}}$ E. Monteil, ${ }^{80 \mathrm{a}, 80 \mathrm{~b}}$ M. Monteno, ${ }^{80 \mathrm{a}}$ M. M. Obertino,${ }^{80 \mathrm{a}, 80 \mathrm{~b}}$ G. Ortona, ${ }^{80 \mathrm{a}, 80 \mathrm{~b}}$ L. Pacher ${ }^{80 \mathrm{a}, 80 \mathrm{~b}}$ N. Pastrone, ${ }^{80 \mathrm{a}}$ M. Pelliccioni, ${ }^{80 \mathrm{a}}$ G. L. Pinna Angioni, ${ }^{80 a, 80 b}$ A. Romero, ${ }^{80,80 b}$ M. Ruspa,${ }^{80 a, 80 c}$ R. Salvatico, ${ }^{80 a, 80 b}$ V. Sola, ${ }^{80 a}$ A. Solano, ${ }^{80 a, 80 b}$ D. Soldi, ${ }^{80 a, 80 b}$ A. Staiano, ${ }^{80 a}$ D. Trocino, ${ }^{80 a, 80 b}$ S. Belforte, ${ }^{81 a}$ V. Candelise,${ }^{81 a, 81 b}$ M. Casarsa, ${ }^{81 a}$ F. Cossutti ${ }^{81 a}$ A. Da Rold, ${ }^{81 a, 81 b}$ G. Della Ricca, ${ }^{81 \mathrm{a}, 81 \mathrm{~b}}$ F. Vazzoler, ${ }^{81,81 b}$ A. Zanetti, ${ }^{81 \mathrm{a}}$ B. Kim, ${ }^{82}$ D. H. Kim, ${ }^{82}$ G. N. Kim, ${ }^{82}$ J. Lee, ${ }^{82}$ S. W. Lee, ${ }^{82}$ C. S. Moon, ${ }^{82}$ Y. D. Oh, ${ }^{82}$ S. I. Pak, ${ }^{82}$ S. Sekmen, ${ }^{82}$ D. C. Son ${ }^{82}$ Y. C. Yang, ${ }^{82}$ H. Kim, ${ }^{83}$ D. H. Moon, ${ }^{83}$ G. Oh, ${ }^{83}$ B. Francois, ${ }^{84}$ T. J. Kim, ${ }^{84}$ J. Park, ${ }^{84}$ S. Cho, ${ }^{85}$ S. Choi,${ }^{85}$ Y. Go, ${ }^{85}$ S. Ha ${ }^{85}$ B. Hong,${ }^{85}$ K. Lee, ${ }^{85}$ K. S. Lee,${ }^{85}$ J. Lim, ${ }^{85}$ J. Park, ${ }^{85}$ S. K. Park, ${ }^{85}$ Y. Roh, ${ }^{85}$ J. Yoo, ${ }^{85}$ J. Goh, ${ }^{86}$ H. S. Kim, ${ }^{87}$ J. Almond, ${ }^{88}$ J. H. Bhyun, ${ }^{88}$ J. Choi, ${ }^{88}$ S. Jeon, ${ }^{88}$ J. Kim, ${ }^{88}$ J. S. Kim,${ }^{88}$ H. Lee, ${ }^{88}$ K. Lee, ${ }^{88}$ S. Lee,${ }^{88}$ K. Nam,${ }^{88}$ M. Oh, ${ }^{88}$ S. B. Oh, ${ }^{88}$ B. C. Radburn-Smith, ${ }^{88}$ U. K. Yang, ${ }^{88}$ H. D. Yoo, ${ }^{88}$ I. Yoon, ${ }^{88}$ D. Jeon, ${ }^{89}$ J. H. Kim, ${ }^{89}$ J. S. H. Lee, ${ }^{89}$ I. C. Park, ${ }^{89}$ I. J. Watson, ${ }^{89}$ Y. Choi,${ }^{90}$ C. Hwang, ${ }^{90}$ Y. Jeong, ${ }^{90}$ J. Lee, ${ }^{90}$ Y. Lee, ${ }^{90}$ I. Yu, ${ }^{90}$ V. Veckalns, ${ }^{91, i i}$ V. Dudenas, ${ }^{92}$ A. Juodagalvis, ${ }^{92}$ A. Rinkevicius, ${ }^{92}$ G. Tamulaitis, ${ }^{92}$ J. Vaitkus ${ }^{92}$ Z. A. Ibrahim, ${ }^{93}$ F. Mohamad Idris, ${ }^{93, j j}$ W. A. T. Wan Abdullah, ${ }^{93}$ M. N. Yusli, ${ }^{93}$ Z. Zolkapli, ${ }^{93}$ J. F. Benitez, ${ }^{94}$ A. Castaneda Hernandez, ${ }^{94}$ J. A. Murillo Quijada, ${ }^{94}$ L. Valencia Palomo, ${ }^{94}$ H. Castilla-Valdez, ${ }^{95}$ E. De La Cruz-Burelo, ${ }^{95}$ I. Heredia-De La Cruz, ${ }^{95, k k}$ R. Lopez-Fernandez, ${ }^{95}$ A. Sanchez-Hernandez, ${ }^{95}$ S. Carrillo Moreno, ${ }^{96}$ C. Oropeza Barrera, ${ }^{96}$ M. Ramirez-Garcia, ${ }^{96}$ F. Vazquez Valencia, ${ }^{96}$ J. Eysermans, ${ }^{97}$ I. Pedraza,${ }^{97}$ H. A. Salazar Ibarguen, ${ }^{97}$ C. Uribe Estrada,${ }^{97}$ A. Morelos Pineda, ${ }^{98}$ J. Mijuskovic, ${ }^{99, \mathrm{c}}$ N. Raicevic, ${ }^{99}$ D. Krofcheck, ${ }^{100}$ S. Bheesette, ${ }^{101}$ P. H. Butler, ${ }^{101}$ A. Ahmad, ${ }^{102}$ M. Ahmad, ${ }^{102}$ Q. Hassan, ${ }^{102}$ H. R. Hoorani, ${ }^{102}$ W. A. Khan, ${ }^{102}$ M. A. Shah,${ }^{102}$ M. Shoaib, ${ }^{102}$ M. Waqas, ${ }^{102}$ V. Avati, ${ }^{103}$ L. Grzanka, ${ }^{103}$ M. Malawski, ${ }^{103}$ H. Bialkowska, ${ }^{104}$ M. Bluj, ${ }^{104}$ B. Boimska, ${ }^{104}$ M. Górski, ${ }^{104}$ M. Kazana,${ }^{104}$ M. Szleper, ${ }^{104}$ P. Zalewski, ${ }^{104}$ K. Bunkowski, ${ }^{105}$ A. Byszuk, ${ }^{105,11}$ K. Doroba ${ }^{105}$ A. Kalinowski, ${ }^{105}$ M. Konecki, ${ }^{105}$ J. Krolikowski, ${ }^{105}$ M. Olszewski, ${ }^{105}$ M. Walczak, ${ }^{105}$ M. Araujo, ${ }^{106}$ P. Bargassa, ${ }^{106}$ D. Bastos, ${ }^{106}$ A. Di Francesco, ${ }^{106}$ P. Faccioli,,${ }^{106}$ B. Galinhas, ${ }^{106}$ M. Gallinaro, ${ }^{106}$ J. Hollar, ${ }^{106}$ N. Leonardo, ${ }^{106}$ T. Niknejad, ${ }^{106}$ J. Seixas, ${ }^{106}$ K. Shchelina, ${ }^{106}$ G. Strong, ${ }^{106}$ O. Toldaiev, ${ }^{106}$ J. Varela, ${ }^{106}$ S. Afanasiev,${ }^{107}$ P. Bunin, ${ }^{107}$ M. Gavrilenko, ${ }^{107}$ I. Golutvin, ${ }^{107}$ I. Gorbunov, ${ }^{107}$ A. Kamenev, ${ }^{107}$ V. Karjavine, ${ }^{107}$ A. Lanev, ${ }^{107}$ A. Malakhov, ${ }^{107}$ V. Matveev, ${ }^{107, \mathrm{~mm}, \mathrm{nn}}$ P. Moisenz, ${ }^{107}$ V. Palichik, ${ }^{107}$ V. Perelygin, ${ }^{107}$ M. Savina, ${ }^{107}$ S. Shmatov, ${ }^{107}$ S. Shulha, ${ }^{107}$ N. Skatchkov, ${ }^{107}$ V. Smirnov,${ }^{107}$ N. Voytishin, ${ }^{107}$ A. Zarubin, ${ }^{107}$

L. Chtchipounov, ${ }^{108}$ V. Golovtcov, ${ }^{108}$ Y. Ivanov, ${ }^{108}$ V. Kim, ${ }^{108,00}$ E. Kuznetsova, ${ }^{108, p p}$ P. Levchenko, ${ }^{108}$ V. Murzin, ${ }^{108}$ V. Oreshkin, ${ }^{108}$ I. Smirnov, ${ }^{108}$ D. Sosnov, ${ }^{108}$ V. Sulimov, ${ }^{108}$ L. Uvarov, ${ }^{108}$ A. Vorobyev, ${ }^{108}$ Yu. Andreev, ${ }^{109}$ A. Dermenev, ${ }^{109}$ S. Gninenko, ${ }^{109}$ N. Golubev, ${ }^{109}$ A. Karneyeu, ${ }^{109}$ M. Kirsanov,${ }^{109}$ N. Krasnikov, ${ }^{109}$ A. Pashenkov, ${ }^{109}$ D. Tlisov, ${ }^{109}$ A. Toropin, ${ }^{109}$ V. Epshteyn, ${ }^{110}$ V. Gavrilov, ${ }^{110}$ N. Lychkovskaya, ${ }^{110}$ A. Nikitenko, ${ }^{110, q q}$ V. Popov, ${ }^{110}$ I. Pozdnyakov, ${ }^{110}$ G. Safronov, ${ }^{110}$ A. Spiridonov, ${ }^{110}$ A. Stepennov, ${ }^{110}$ M. Toms, ${ }^{110}$ E. Vlasov,${ }^{110}$ A. Zhokin, ${ }^{110}$ T. Aushev,${ }^{111}$ M. Chadeeva, ${ }^{12, \text { rr }}$ P. Parygin, ${ }^{112}$ D. Philippov, ${ }^{112}$ E. Popova, ${ }^{112}$ V. Rusinov, ${ }^{112}$ V. Andreev, ${ }^{113}$ M. Azarkin, ${ }^{113}$ I. Dremin, ${ }^{113}$ M. Kirakosyan, ${ }^{113}$ A. Terkulov, ${ }^{113}$ A. Belyaev, ${ }^{114}$ E. Boos, ${ }^{114}$ V. Bunichev, ${ }^{114}$ M. Dubinin, ${ }^{114, s s}$ L. Dudko, ${ }^{114}$ V. Klyukhin, ${ }^{114}$ O. Kodolova, ${ }^{114}$

I. Lokhtin, ${ }^{114}$ S. Obraztsov, ${ }^{114}$ M. Perfilov, ${ }^{114}$ S. Petrushanko, ${ }^{114}$ V. Savrin, ${ }^{114}$ A. Snigirev, ${ }^{114}$ A. Barnyakov, ${ }^{115, t t}$

V. Blinov, ${ }^{115, t \mathrm{tt}}$ T. Dimova, ${ }^{115, \mathrm{tt}}$ L. Kardapoltsev, ${ }^{115, \mathrm{tt}}$ Y. Skovpen, ${ }^{115, \mathrm{tt}}$ I. Azhgirey, ${ }^{116}$ I. Bayshev, ${ }^{116} \mathrm{~S}$. Bitioukov, ${ }^{116}$ V. Kachanov, ${ }^{116}$ D. Konstantinov, ${ }^{116}$ P. Mandrik, ${ }^{116}$ V. Petrov,${ }^{116}$ R. Ryutin, ${ }^{116}$ S. Slabospitskii, ${ }^{116}$ A. Sobol, ${ }^{116}$ S. Troshin, ${ }^{116}$ N. Tyurin, ${ }^{116}$ A. Uzunian, ${ }^{116}$ A. Volkov, ${ }^{116}$ A. Babaev, ${ }^{117}$ A. Iuzhakov, ${ }^{117}$ V. Okhotnikov, ${ }^{117}$ V. Borchsh, ${ }^{118}$ V. Ivanchenko, ${ }^{118}$ E. Tcherniaev, ${ }^{118}$ P. Adzic, ${ }^{119, \text { uu }}$ P. Cirkovic, ${ }^{119}$ M. Dordevic, ${ }^{119}$ P. Milenovic, ${ }^{119}$ J. Milosevic, ${ }^{119}$ M. Stojanovic, ${ }^{119}$ M. Aguilar-Benitez, ${ }^{120}$ J. Alcaraz Maestre, ${ }^{120}$ A. Álvarez Fernández, ${ }^{120}$ I. Bachiller, ${ }^{120}$ M. Barrio Luna, ${ }^{120}$ Cristina F. Bedoya, ${ }^{120}$ J. A. Brochero Cifuentes, ${ }^{120}$ C. A. Carrillo Montoya,${ }^{120}$ M. Cepeda, ${ }^{120}$ M. Cerrada,${ }^{120}$ N. Colino, ${ }^{120}$ B. De La Cruz, ${ }^{120}$ A. Delgado Peris, ${ }^{120}$ J. P. Fernández Ramos, ${ }^{120}$ J. Flix, ${ }^{120}$ M. C. Fouz, ${ }^{120}$ O. Gonzalez Lopez, ${ }^{12}$ S. Goy Lopez, ${ }^{120}$ J. M. Hernandez, ${ }^{120}$ M. I. Josa ${ }^{120}$ D. Moran, ${ }^{120}$ Á. Navarro Tobar, ${ }^{120}$ A. Pérez-Calero Yzquierdo, ${ }^{120}$ J. Puerta Pelayo, ${ }^{120}$ I. Redondo, ${ }^{120}$ L. Romero, ${ }^{120}$ S. Sánchez Navas, ${ }^{120}$ M. S. Soares, ${ }^{120}$ A. Triossi, ${ }^{120}$ C. Willmott, ${ }^{120}$ C. Albajar, ${ }^{121}$ J. F. de Trocóniz, ${ }^{121}$ R. Reyes-Almanza, ${ }^{121}$ B. Alvarez Gonzalez, ${ }^{122}$ J. Cuevas, ${ }^{122}$ C. Erice, ${ }^{122}$ J. Fernandez Menendez, ${ }^{122}$ S. Folgueras, ${ }^{122}$ I. Gonzalez Caballero, ${ }^{122}$ J. R. González Fernández ${ }^{122}$ E. Palencia Cortezon, ${ }^{122}$ V. Rodríguez Bouza, ${ }^{122}$ S. Sanchez Cruz, ${ }^{122}$ I. J. Cabrillo, ${ }^{123}$ A. Calderon, ${ }^{123}$ B. Chazin Quero, ${ }^{123}$ J. Duarte Campderros, ${ }^{123}$ 
M. Fernandez, ${ }^{123}$ P. J. Fernández Manteca, ${ }^{123}$ A. García Alonso, ${ }^{123}$ G. Gomez, ${ }^{123}$ C. Martinez Rivero, ${ }^{123}$ P. Martinez Ruiz del Arbol, ${ }^{123}$ F. Matorras, ${ }^{123}$ J. Piedra Gomez, ${ }^{123}$ C. Prieels, ${ }^{123}$ T. Rodrigo, ${ }^{123}$ A. Ruiz-Jimeno, ${ }^{123}$ L. Russo, ${ }^{123, v v}$ L. Scodellaro, ${ }^{123}$ I. Vila, ${ }^{123}$ J. M. Vizan Garcia, ${ }^{123}$ K. Malagalage, ${ }^{124}$ W. G. D. Dharmaratna, ${ }^{125}$ N. Wickramage, ${ }^{125}$ D. Abbaneo, ${ }^{126}$ B. Akgun, ${ }^{126}$ E. Auffray, ${ }^{126}$ G. Auzinger, ${ }^{126}$ J. Baechler, ${ }^{126}$ P. Baillon, ${ }^{126}$ A. H. Ball, ${ }^{126}$ D. Barney, ${ }^{126}$ J. Bendavid, ${ }^{126}$ M. Bianco, ${ }^{126}$ A. Bocci, ${ }^{126}$ P. Bortignon, ${ }^{126}$ E. Bossini, ${ }^{126}$ C. Botta, ${ }^{126}$ E. Brondolin, ${ }^{126}$ T. Camporesi, ${ }^{126}$ A. Caratelli, ${ }^{126}$ G. Cerminara, ${ }^{126}$ E. Chapon, ${ }^{126}$ G. Cucciati, ${ }^{126}$ D. d'Enterria, ${ }^{126}$ A. Dabrowski, ${ }^{126}$ N. Daci, ${ }^{126}$ V. Daponte, ${ }^{126}$ A. David, ${ }^{126}$ O. Davignon, ${ }^{126}$ A. De Roeck, ${ }^{126}$ M. Deile,${ }^{126}$ M. Dobson, ${ }^{126}$ M. Dünser, ${ }^{126}$ N. Dupont, ${ }^{126}$ A. Elliott-Peisert, ${ }^{126}$ N. Emriskova, ${ }^{126}$ F. Fallavollita, ${ }^{126, w w}$ D. Fasanella, ${ }^{126}$ S. Fiorendi, ${ }^{126}$ G. Franzoni, ${ }^{126}$ J. Fulcher, ${ }^{126}$ W. Funk, ${ }^{126}$ S. Giani, ${ }^{126}$ D. Gigi, ${ }^{126}$ A. Gilbert, ${ }^{126}$ K. Gill, ${ }^{126}$ F. Glege, ${ }^{126}$ L. Gouskos, ${ }^{126}$ M. Gruchala, ${ }^{126}$ M. Guilbaud, ${ }^{126}$ D. Gulhan, ${ }^{126}$ J. Hegeman, ${ }^{126}$ C. Heidegger, ${ }^{126}$ Y. Iiyama, ${ }^{126}$ V. Innocente, ${ }^{126}$ T. James, ${ }^{126}$ P. Janot, ${ }^{126}$

O. Karacheban, ${ }^{126, u}$ J. Kaspar, ${ }^{126}$ J. Kieseler, ${ }^{126}$ M. Krammer, ${ }^{126, b}$ N. Kratochwil, ${ }^{126}$ C. Lange, ${ }^{126}$ P. Lecoq, ${ }^{126}$ C. Lourenço, ${ }^{126}$ L. Malgeri, ${ }^{126}$ M. Mannelli, ${ }^{126}$ A. Massironi, ${ }^{126}$ F. Meijers, ${ }^{126}$ S. Mersi, ${ }^{126}$ E. Meschi, ${ }^{126}$ F. Moortgat, ${ }^{126}$ M. Mulders, ${ }^{126}$ J. Ngadiuba, ${ }^{126}$ J. Niedziela, ${ }^{126}$ S. Nourbakhsh, ${ }^{126}$ S. Orfanelli, ${ }^{126}$ L. Orsini, ${ }^{126}$ F. Pantaleo, ${ }^{126, r}$ L. Pape, ${ }^{126}$ E. Perez, ${ }^{126}$ M. Peruzzi, ${ }^{126}$ A. Petrilli, ${ }^{126}$ G. Petrucciani, ${ }^{126}$ A. Pfeiffer, ${ }^{126}$ M. Pierini, ${ }^{126}$ F. M. Pitters, ${ }^{126}$ D. Rabady, ${ }^{126}$ A. Racz, ${ }^{126}$ M. Rieger, ${ }^{126}$ M. Rovere, ${ }^{126}$ H. Sakulin, ${ }^{126}$ J. Salfeld-Nebgen, ${ }^{126}$ C. Schäfer, ${ }^{126}$ C. Schwick, ${ }^{126}$ M. Selvaggi, ${ }^{126}$ A. Sharma, ${ }^{126}$ P. Silva, ${ }^{126}$ W. Snoeys, ${ }^{126}$ P. Sphicas, ${ }^{126, x x}$ J. Steggemann, ${ }^{126}$ S. Summers, ${ }^{126}$ V. R. Tavolaro, ${ }^{126}$ D. Treille, ${ }^{126}$ A. Tsirou, ${ }^{126}$ G. P. Van Onsem, ${ }^{126}$ A. Vartak, ${ }^{126}$ M. Verzetti, ${ }^{126}$ W. D. Zeuner, ${ }^{126}$ L. Caminada, ${ }^{127, y y}$ K. Deiters, ${ }^{127}$ W. Erdmann, ${ }^{127}$ R. Horisberger, ${ }^{127}$ Q. Ingram, ${ }^{127}$ H. C. Kaestli, ${ }^{127}$ D. Kotlinski, ${ }^{127}$ U. Langenegger, ${ }^{127}$ T. Rohe, ${ }^{127}$ S. A. Wiederkehr, ${ }^{127}$ M. Backhaus, ${ }^{128}$ P. Berger, ${ }^{128}$ N. Chernyavskaya, ${ }^{128}$ G. Dissertori, ${ }^{128}$ M. Dittmar, ${ }^{128}$ M. Donegà, ${ }^{128}$ C. Dorfer, ${ }^{128}$ T. A. Gómez Espinosa, ${ }^{128}$ C. Grab, ${ }^{128}$ D. Hits, ${ }^{128}$ W. Lustermann, ${ }^{128}$ R. A. Manzoni, ${ }^{128}$ M. T. Meinhard, ${ }^{128}$ F. Micheli, ${ }^{128}$ P. Musella, ${ }^{128}$ F. Nessi-Tedaldi, ${ }^{128}$ F. Pauss, ${ }^{128}$ G. Perrin, ${ }^{128}$ L. Perrozzi, ${ }^{128}$ S. Pigazzini, ${ }^{128}$ M. G. Ratti, ${ }^{128}$ M. Reichmann, ${ }^{128}$ C. Reissel, ${ }^{128}$ T. Reitenspiess, ${ }^{128}$ B. Ristic, ${ }^{128}$ D. Ruini, ${ }^{128}$ D. A. Sanz Becerra, ${ }^{128}$ M. Schönenberger, ${ }^{128}$

L. Shchutska, ${ }^{128}$ M. L. Vesterbacka Olsson, ${ }^{128}$ R. Wallny, ${ }^{128}$ D. H. Zhu, ${ }^{128}$ T. K. Aarrestad, ${ }^{129}$ C. Amsler, ${ }^{129, z z}$

D. Brzhechko, ${ }^{129}$ M. F. Canelli, ${ }^{129}$ A. De Cosa, ${ }^{129}$ R. Del Burgo, ${ }^{129}$ B. Kilminster, ${ }^{129}$ S. Leontsinis, ${ }^{129}$ V. M. Mikuni, ${ }^{129}$ I. Neutelings, ${ }^{129}$ G. Rauco, ${ }^{129}$ P. Robmann, ${ }^{129}$ K. Schweiger, ${ }^{129}$ C. Seitz, ${ }^{129}$ Y. Takahashi, ${ }^{129}$ S. Wertz, ${ }^{129}$ A. Zucchetta, ${ }^{129}$ T. H. Doan, ${ }^{130}$ C. M. Kuo, ${ }^{130}$ W. Lin, ${ }^{130}$ A. Roy, ${ }^{130}$ S. S. Yu, ${ }^{130}$ P. Chang, ${ }^{131}$ Y. Chao, ${ }^{131}$ K. F. Chen, ${ }^{131}$ P. H. Chen, ${ }^{131}$ W.-S. Hou, ${ }^{131}$ Y. y. Li, ${ }^{131}$ R.-S. Lu, ${ }^{131}$ E. Paganis, ${ }^{131}$ A. Psallidas, ${ }^{131}$ A. Steen, ${ }^{131}$ B. Asavapibhop, ${ }^{132}$

C. Asawatangtrakuldee, ${ }^{132}$ N. Srimanobhas, ${ }^{132}$ N. Suwonjandee, ${ }^{132}$ A. Bat, ${ }^{133}$ F. Boran, ${ }^{133}$ A. Celik, ${ }^{133, \text { aaa }}$ S. Cerci, ${ }^{133, b b b}$ S. Damarseckin, ${ }^{133, \text { ccc }}$ Z. S. Demiroglu, ${ }^{133}$ F. Dolek, ${ }^{133}$ C. Dozen, ${ }^{133, \text { ddd }}$ I. Dumanoglu, ${ }^{133}$ G. Gokbulut, ${ }^{133}$ Emine Gurpinar Guler, ${ }^{133, \text { eee }}$ Y. Guler, ${ }^{133}$ I. Hos, ${ }^{133, f f f}$ C. Isik, ${ }^{133}$ E. E. Kangal, ${ }^{133, \text { ggg }}$ O. Kara, ${ }^{133}$ A. Kayis Topaksu, ${ }^{133}$ U. Kiminsu, ${ }^{133}$ G. Onengut, ${ }^{133}$ K. Ozdemir, ${ }^{133, \text { hhh }}$ S. Ozturk, ${ }^{133, \text { iii }}$ A. E. Simsek, ${ }^{133}$ D. Sunar Cerci, ${ }^{133, \text { bbb }}$ U. G. Tok, ${ }^{133}$ S. Turkcapar, ${ }^{133}$ I. S. Zorbakir, ${ }^{133}$ C. Zorbilmez, ${ }^{133}$ B. Isildak, ${ }^{134, j j j}$ G. Karapinar, ${ }^{134, k k k}$ M. Yalvac, ${ }^{134}$ I. O. Atakisi, ${ }^{135}$ E. Gülmez, ${ }^{135}$ M. Kaya, ${ }^{135,111}$ O. Kaya, ${ }^{135, m m m}$ Ö. Özçelik, ${ }^{135}$ S. Tekten, ${ }^{135}$ E. A. Yetkin, ${ }^{135, \text { nnn }}$ A. Cakir, ${ }^{136}$ K. Cankocak, ${ }^{136}$ Y. Komurcu, ${ }^{136}$ S. Sen, ${ }^{136,000}$ B. Kaynak, ${ }^{137}$ S. Ozkorucuklu, ${ }^{137}$ B. Grynyov, ${ }^{138}$ L. Levchuk, ${ }^{139}$ E. Bhal, ${ }^{140}$ S. Bologna, ${ }^{140}$ J. J. Brooke, ${ }^{140}$ D. Burns, ${ }^{140, p p p}$ E. Clement, ${ }^{140}$ D. Cussans, ${ }^{140}$ H. Flacher, ${ }^{140}$ J. Goldstein, ${ }^{140}$ G. P. Heath, ${ }^{140}$ H. F. Heath, ${ }^{140}$ L. Kreczko, ${ }^{140}$ B. Krikler, ${ }^{140}$ S. Paramesvaran, ${ }^{140}$ B. Penning, ${ }^{140}$ T. Sakuma, ${ }^{140}$ S. Seif El Nasr-Storey, ${ }^{140}$ V. J. Smith, ${ }^{140}$ J. Taylor, ${ }^{140}$ A. Titterton, ${ }^{140}$ K. W. Bell, ${ }^{141}$ A. Belyaev, ${ }^{141, q q q}$ C. Brew, ${ }^{141}$ R. M. Brown, ${ }^{141}$ D. J. A. Cockerill, ${ }^{141}$ J. A. Coughlan, ${ }^{141}$ K. Harder, ${ }^{141}$ S. Harper, ${ }^{141}$ J. Linacre, ${ }^{141}$ K. Manolopoulos, ${ }^{141}$ D. M. Newbold, ${ }^{141}$ E. Olaiya, ${ }^{141}$ D. Petyt, ${ }^{141}$ T. Reis, ${ }^{141}$ T. Schuh, ${ }^{141}$ C. H. Shepherd-Themistocleous, ${ }^{141}$ A. Thea, ${ }^{141}$ I. R. Tomalin, ${ }^{141}$ T. Williams, ${ }^{141}$

W. J. Womersley, ${ }^{141}$ R. Bainbridge, ${ }^{142}$ P. Bloch, ${ }^{142}$ J. Borg, ${ }^{142}$ S. Breeze, ${ }^{142}$ O. Buchmuller, ${ }^{142}$ A. Bundock, ${ }^{142}$ Gurpreet Singh Chahal, ${ }^{142, \mathrm{rrr}}$ D. Colling, ${ }^{142}$ P. Dauncey, ${ }^{142}$ G. Davies, ${ }^{142}$ M. Della Negra, ${ }^{142}$ R. Di Maria, ${ }^{142}$ P. Everaerts, ${ }^{142}$ G. Hall, ${ }^{142}$ G. Iles, ${ }^{142}$ M. Komm, ${ }^{142}$ C. Laner, ${ }^{142}$ L. Lyons, ${ }^{142}$ A.-M. Magnan, ${ }^{142}$ S. Malik, ${ }^{142}$ A. Martelli, ${ }^{142}$ V. Milosevic, ${ }^{142}$ A. Morton, ${ }^{142}$ J. Nash, ${ }^{142, s s s}$ V. Palladino, ${ }^{142}$ M. Pesaresi, ${ }^{142}$ D. M. Raymond, ${ }^{142}$ A. Richards, ${ }^{142}$ A. Rose, ${ }^{142}$ E. Scott, ${ }^{142}$ C. Seez, ${ }^{142}$ A. Shtipliyski, ${ }^{142}$ M. Stoye, ${ }^{142}$ T. Strebler, ${ }^{142}$ A. Tapper, ${ }^{142}$ K. Uchida, ${ }^{142}$ T. Virdee, ${ }^{142, r}$ N. Wardle, ${ }^{142}$ D. Winterbottom, ${ }^{142}$ J. Wright, ${ }^{142}$ A. G. Zecchinelli, ${ }^{142}$ S. C. Zenz, ${ }^{142}$ J. E. Cole, ${ }^{143}$ P. R. Hobson, ${ }^{143}$ A. Khan, ${ }^{143}$ P. Kyberd, ${ }^{143}$ C. K. Mackay, ${ }^{143}$ I. D. Reid, ${ }^{143}$ L. Teodorescu, ${ }^{143}$ S. Zahid, ${ }^{143}$ K. Call, ${ }^{144}$ B. Caraway, ${ }^{144}$ J. Dittmann, ${ }^{144}$ K. Hatakeyama, ${ }^{144}$ C. Madrid, ${ }^{144}$ B. McMaster, ${ }^{144}$ N. Pastika, ${ }^{144}$ C. Smith, ${ }^{144}$ R. Bartek, ${ }^{145}$ A. Dominguez, ${ }^{145}$ R. Uniyal, ${ }^{145}$ A. M. Vargas Hernandez, ${ }^{145}$ A. Buccilli, ${ }^{146}$ S. I. Cooper, ${ }^{146}$ C. Henderson, ${ }^{146}$ P. Rumerio, ${ }^{146}$ C. West, ${ }^{146}$ A. Albert, ${ }^{147}$ D. Arcaro, ${ }^{147}$ Z. Demiragli, ${ }^{147}$ D. Gastler, ${ }^{147}$ C. Richardson, ${ }^{147}$ J. Rohlf, ${ }^{147}$ D. Sperka, ${ }^{147}$ I. Suarez, ${ }^{147}$ L. Sulak, ${ }^{147}$ D. Zou, ${ }^{147}$ 
G. Benelli, ${ }^{148}$ B. Burkle, ${ }^{148}$ X. Coubez, ${ }^{148, s}$ D. Cutts, ${ }^{148}$ Y. t. Duh, ${ }^{148}$ M. Hadley, ${ }^{148}$ U. Heintz, ${ }^{148}$ J. M. Hogan, ${ }^{148, t t t}$ K. H. M. Kwok, ${ }^{148}$ E. Laird, ${ }^{148}$ G. Landsberg, ${ }^{148}$ K. T. Lau, ${ }^{148}$ J. Lee, ${ }^{148}$ Z. Mao, ${ }^{148}$ M. Narain, ${ }^{148}$ S. Sagir, ${ }^{148, \text { uuu }}$ R. Syarif, ${ }^{148}$ E. Usai, ${ }^{148}$ W. Y. Wong, ${ }^{148}$ D. Yu, ${ }^{148}$ W. Zhang, ${ }^{148}$ R. Band, ${ }^{149}$ C. Brainerd, ${ }^{149}$ R. Breedon, ${ }^{149}$

M. Calderon De La Barca Sanchez, ${ }^{149}$ M. Chertok, ${ }^{149}$ J. Conway, ${ }^{149}$ R. Conway, ${ }^{149}$ P. T. Cox, ${ }^{149}$ R. Erbacher, ${ }^{149}$ C. Flores, ${ }^{149}$ G. Funk, ${ }^{149}$ F. Jensen, ${ }^{149}$ W. Ko, ${ }^{149}$ O. Kukral, ${ }^{149}$ R. Lander, ${ }^{149}$ M. Mulhearn, ${ }^{149}$ D. Pellett, ${ }^{149}$ J. Pilot, ${ }^{149}$ M. Shi, ${ }^{149}$ D. Taylor, ${ }^{149}$ K. Tos, ${ }^{149}$ M. Tripathi, ${ }^{149}$ Z. Wang, ${ }^{149}$ F. Zhang, ${ }^{149}$ M. Bachtis, ${ }^{150}$ C. Bravo, ${ }^{150}$ R. Cousins, ${ }^{150}$ A. Dasgupta, ${ }^{150}$ A. Florent, ${ }^{150}$ J. Hauser, ${ }^{150}$ M. Ignatenko, ${ }^{150}$ N. Mccoll, ${ }^{150}$ W. A. Nash, ${ }^{150}$ S. Regnard, ${ }^{150}$ D. Saltzberg, ${ }^{150}$ C. Schnaible, ${ }^{150}$ B. Stone, ${ }^{150}$ V. Valuev, ${ }^{150}$ K. Burt, ${ }^{151}$ Y. Chen, ${ }^{151}$ R. Clare, ${ }^{151}$ J. W. Gary, ${ }^{151}$ S. M. A. Ghiasi Shirazi, ${ }^{151}$ G. Hanson, ${ }^{151}$ G. Karapostoli, ${ }^{151}$ E. Kennedy, ${ }^{151}$ O. R. Long, ${ }^{151}$ M. Olmedo Negrete, ${ }^{151}$ M. I. Paneva, ${ }^{151}$ W. Si, ${ }^{151}$ L. Wang, ${ }^{151}$ S. Wimpenny, ${ }^{151}$ B. R. Yates, ${ }^{151}$ Y. Zhang, ${ }^{151}$ J. G. Branson, ${ }^{152}$ P. Chang, ${ }^{152}$ S. Cittolin, ${ }^{152}$ S. Cooperstein, ${ }^{152}$ N. Deelen, ${ }^{152}$ M. Derdzinski, ${ }^{152}$ R. Gerosa, ${ }^{152}$ D. Gilbert, ${ }^{152}$ B. Hashemi, ${ }^{152}$ D. Klein, ${ }^{152}$ V. Krutelyov, ${ }^{152}$ J. Letts, ${ }^{152}$ M. Masciovecchio, ${ }^{152}$

S. May, ${ }^{152}$ S. Padhi, ${ }^{152}$ M. Pieri, ${ }^{152}$ V. Sharma, ${ }^{152}$ M. Tadel, ${ }^{152}$ F. Würthwein, ${ }^{152}$ A. Yagil, ${ }^{152}$ G. Zevi Della Porta, ${ }^{152}$ N. Amin, ${ }^{153}$ R. Bhandari, ${ }^{153}$ C. Campagnari, ${ }^{153}$ M. Citron, ${ }^{153}$ V. Dutta, ${ }^{153}$ M. Franco Sevilla, ${ }^{153}$ J. Incandela, ${ }^{153}$ B. Marsh, ${ }^{153}$ H. Mei, ${ }^{153}$ A. Ovcharova, ${ }^{153}$ H. Qu, ${ }^{153}$ J. Richman, ${ }^{153}$ U. Sarica, ${ }^{153}$ D. Stuart, ${ }^{153}$ S. Wang, ${ }^{153}$ D. Anderson, ${ }^{154}$ A. Bornheim, ${ }^{154}$ O. Cerri, ${ }^{154}$ I. Dutta, ${ }^{154}$ J. M. Lawhorn, ${ }^{154}$ N. Lu, ${ }^{154}$ J. Mao, ${ }^{154}$ H. B. Newman, ${ }^{154}$ T. Q. Nguyen, ${ }^{154}$ J. Pata, ${ }^{154}$ M. Spiropulu, ${ }^{154}$ J. R. Vlimant, ${ }^{154}$ S. Xie, ${ }^{154}$ Z. Zhang, ${ }^{154}$ R. Y. Zhu, ${ }^{154}$ M. B. Andrews, ${ }^{155}$ T. Ferguson, ${ }^{155}$ T. Mudholkar, ${ }^{155}$ M. Paulini, ${ }^{155}$ M. Sun, ${ }^{155}$ I. Vorobiev, ${ }^{155}$ M. Weinberg, ${ }^{155}$ J. P. Cumalat, ${ }^{156}$ W. T. Ford, ${ }^{156}$ E. MacDonald, ${ }^{156}$ T. Mulholland, ${ }^{156}$ R. Patel, ${ }^{156}$ A. Perloff, ${ }^{156}$ K. Stenson, ${ }^{156}$ K. A. Ulmer, ${ }^{156}$ S. R. Wagner, ${ }^{156}$ J. Alexander, ${ }^{157}$ Y. Cheng, ${ }^{157}$ J. Chu, ${ }^{157}$ A. Datta, ${ }^{157}$ A. Frankenthal, ${ }^{157}$ K. Mcdermott, ${ }^{157}$ J. R. Patterson, ${ }^{157}$ D. Quach, ${ }^{157}$ A. Ryd, ${ }^{157}$ S. M. Tan, ${ }^{157}$ Z. Tao, ${ }^{157}$ J. Thom, ${ }^{157}$ P. Wittich, ${ }^{157}$ M. Zientek, ${ }^{157}$ S. Abdullin, ${ }^{158}$ M. Albrow, ${ }^{158}$ M. Alyari, ${ }^{158}$ G. Apollinari, ${ }^{158}$ A. Apresyan, ${ }^{158}$ A. Apyan, ${ }^{158}$ S. Banerjee, ${ }^{158}$ L. A. T. Bauerdick, ${ }^{158}$ A. Beretvas, ${ }^{158}$ D. Berry, ${ }^{158}$ J. Berryhill, ${ }^{158}$ P. C. Bhat, ${ }^{158}$ K. Burkett, ${ }^{158}$ J. N. Butler, ${ }^{158}$ A. Canepa, ${ }^{158}$ G. B. Cerati, ${ }^{158}$ H. W. K. Cheung, ${ }^{158}$ F. Chlebana, ${ }^{158}$ M. Cremonesi, ${ }^{158}$ J. Duarte, ${ }^{158}$ V. D. Elvira, ${ }^{158}$ J. Freeman, ${ }^{158}$ Z. Gecse, ${ }^{158}$ E. Gottschalk, ${ }^{158}$ L. Gray, ${ }^{158}$ D. Green, ${ }^{158}$ S. Grünendahl, ${ }^{158}$ O. Gutsche, ${ }^{158}$ Allison Reinsvold Hall, ${ }^{158}$ J. Hanlon, ${ }^{158}$ R. M. Harris, ${ }^{158}$ S. Hasegawa, ${ }^{158}$ R. Heller, ${ }^{158}$ J. Hirschauer, ${ }^{158}$ B. Jayatilaka, ${ }^{158}$ S. Jindariani, ${ }^{158}$ M. Johnson, ${ }^{158}$ U. Joshi, ${ }^{158}$ T. Klijnsma, ${ }^{158}$ B. Klima, ${ }^{158}$ M. J. Kortelainen, ${ }^{158}$ B. Kreis, ${ }^{158}$ S. Lammel, ${ }^{158}$ J. Lewis, ${ }^{158}$ D. Lincoln, ${ }^{158}$ R. Lipton, ${ }^{158}$ M. Liu, ${ }^{158}$ T. Liu, ${ }^{158}$ J. Lykken, ${ }^{158}$ K. Maeshima, ${ }^{158}$ J. M. Marraffino, ${ }^{158}$ D. Mason, ${ }^{158}$ P. McBride, ${ }^{158}$ P. Merkel, ${ }^{158}$ S. Mrenna, ${ }^{158}$ S. Nahn, ${ }^{158}$ V. O’Dell, ${ }^{158}$ V. Papadimitriou, ${ }^{158}$ K. Pedro, ${ }^{158}$ C. Pena, ${ }^{158}$ G. Rakness, ${ }^{158}$ F. Ravera, ${ }^{158}$ L. Ristori, ${ }^{158}$ B. Schneider, ${ }^{158}$ E. Sexton-Kennedy, ${ }^{158}$ N. Smith, ${ }^{158}$ A. Soha, ${ }^{158}$ W. J. Spalding, ${ }^{158}$ L. Spiegel, ${ }^{158}$ S. Stoynev, ${ }^{158}$ J. Strait, ${ }^{158}$ N. Strobbe, ${ }^{158}$ L. Taylor, ${ }^{158}$ S. Tkaczyk, ${ }^{158}$ N. V. Tran, ${ }^{158}$ L. Uplegger, ${ }^{158}$ E. W. Vaandering, ${ }^{158}$ C. Vernieri, ${ }^{158}$ R. Vidal, ${ }^{158}$ M. Wang, ${ }^{158}$ H. A. Weber, ${ }^{158}$ D. Acosta, ${ }^{159}$ P. Avery, ${ }^{159}$ D. Bourilkov, ${ }^{159}$ A. Brinkerhoff, ${ }^{159}$ L. Cadamuro, ${ }^{159}$ A. Carnes, ${ }^{159}$ V. Cherepanov, ${ }^{159}$ F. Errico, ${ }^{159}$ R. D. Field, ${ }^{159}$ S. V. Gleyzer, ${ }^{159}$ D. Guerrero, ${ }^{159}$ B. M. Joshi, ${ }^{159}$ M. Kim, ${ }^{159}$ J. Konigsberg, ${ }^{159}$ A. Korytov, ${ }^{159}$ K. H. Lo, ${ }^{159}$ P. Ma, ${ }^{159}$ K. Matchev, ${ }^{159}$ N. Menendez, ${ }^{159}$ G. Mitselmakher, ${ }^{159}$ D. Rosenzweig, ${ }^{159}$ K. Shi, ${ }^{159}$ J. Wang, ${ }^{159}$ S. Wang, ${ }^{159}$ X. Zuo, ${ }^{159}$ Y. R. Joshi, ${ }^{160}$ T. Adams, ${ }^{161}$ A. Askew, ${ }^{161}$ S. Hagopian, ${ }^{161}$ V. Hagopian, ${ }^{161}$ K. F. Johnson, ${ }^{161}$ R. Khurana, ${ }^{161}$ T. Kolberg, ${ }^{161}$ G. Martinez, ${ }^{161}$ T. Perry, ${ }^{161}$ H. Prosper, ${ }^{161}$ C. Schiber, ${ }^{161}$ R. Yohay, ${ }^{161}$ J. Zhang, ${ }^{161}$ M. M. Baarmand, ${ }^{162}$ M. Hohlmann, ${ }^{162}$ D. Noonan, ${ }^{162}$ M. Rahmani, ${ }^{162}$ M. Saunders, ${ }^{162}$ F. Yumiceva, ${ }^{162}$ M. R. Adams, ${ }^{163}$ L. Apanasevich, ${ }^{163}$ R. R. Betts, ${ }^{163}$ R. Cavanaugh, ${ }^{163}$ X. Chen, ${ }^{163}$ S. Dittmer, ${ }^{163}$ O. Evdokimov, ${ }^{163}$ C. E. Gerber, ${ }^{163}$ D. A. Hangal, ${ }^{163}$ D. J. Hofman, ${ }^{163}$ K. Jung, ${ }^{163}$ C. Mills, ${ }^{163}$ T. Roy, ${ }^{163}$ M. B. Tonjes, ${ }^{163}$ N. Varelas, ${ }^{163}$ J. Viinikainen, ${ }^{163}$ H. Wang, ${ }^{163}$ X. Wang, ${ }^{163}$ Z. Wu, ${ }^{163}$ M. Alhusseini, ${ }^{164}$ B. Bilki, ${ }^{164, e e e}$ W. Clarida, ${ }^{164}$ K. Dilsiz, ${ }^{164, v v v}$ S. Durgut, ${ }^{164}$ R. P. Gandrajula, ${ }^{164}$ M. Haytmyradov, ${ }^{164}$ V. Khristenko, ${ }^{164}$ O. K. Köseyan, ${ }^{164}$ J.-P. Merlo, ${ }^{164}$ A. Mestvirishvili, ${ }^{164, w w w}$ A. Moeller, ${ }^{164}$ J. Nachtman, ${ }^{164}$ H. Ogul, ${ }^{164, x x x}$ Y. Onel, ${ }^{164}$ F. Ozok, ${ }^{164, y y y}$ A. Penzo, ${ }^{164}$ C. Snyder, ${ }^{164}$ E. Tiras, ${ }^{164}$ J. Wetzel, ${ }^{164}$ B. Blumenfeld, ${ }^{165}$ A. Cocoros, ${ }^{165}$ N. Eminizer, ${ }^{165}$ A. V. Gritsan, ${ }^{165}$ W. T. Hung, ${ }^{165}$ S. Kyriacou, ${ }^{165}$ P. Maksimovic, ${ }^{165}$ J. Roskes, ${ }^{165}$ M. Swartz, ${ }^{165}$ C. Baldenegro Barrera, ${ }^{166}$ P. Baringer, ${ }^{166}$ A. Bean, ${ }^{166} \mathrm{~S}$. Boren, ${ }^{166}$ J. Bowen, ${ }^{166}$ A. Bylinkin, ${ }^{166}$ T. Isidori, ${ }^{166} \mathrm{~S}$. Khalil, ${ }^{166}$ J. King, ${ }^{166}$ G. Krintiras, ${ }^{166}$ A. Kropivnitskaya, ${ }^{166}$ C. Lindsey, ${ }^{166}$ D. Majumder, ${ }^{166}$ W. Mcbrayer, ${ }^{166}$ N. Minafra, ${ }^{166}$ M. Murray, ${ }^{166}$ C. Rogan, ${ }^{166}$ C. Royon, ${ }^{166}$ S. Sanders, ${ }^{166}$ E. Schmitz, ${ }^{166}$ J. D. Tapia Takaki, ${ }^{166}$ Q. Wang, ${ }^{166}$ J. Williams, ${ }^{166}$ G. Wilson, ${ }^{166}$ S. Duric, ${ }^{167}$ A. Ivanov, ${ }^{167}$ K. Kaadze, ${ }^{167}$ D. Kim, ${ }^{167}$ Y. Maravin, ${ }^{167}$ D. R. Mendis, ${ }^{167}$ T. Mitchell, ${ }^{167}$ A. Modak, ${ }^{167}$ A. Mohammadi, ${ }^{167}$ F. Rebassoo, ${ }^{168}$ D. Wright, ${ }^{168}$ A. Baden, ${ }^{169}$ O. Baron, ${ }^{169}$ A. Belloni, ${ }^{169}$ S. C. Eno, ${ }^{169}$ Y. Feng, ${ }^{169}$ N. J. Hadley, ${ }^{169}$ S. Jabeen, ${ }^{169}$ G. Y. Jeng, ${ }^{169}$ R. G. Kellogg, ${ }^{169}$ J. Kunkle, ${ }^{169}$ A. C. Mignerey, ${ }^{169}$ S. Nabili, ${ }^{169}$ F. Ricci-Tam, ${ }^{169}$ M. Seidel, ${ }^{169}$ Y. H. Shin, ${ }^{169}$ A. Skuja, ${ }^{169}$ S. C. Tonwar, ${ }^{169}$ K. Wong, ${ }^{169}$ D. Abercrombie, ${ }^{170}$ B. Allen, ${ }^{170}$ 
A. Baty, ${ }^{170}$ R. Bi, ${ }^{170}$ S. Brandt, ${ }^{170}$ W. Busza, ${ }^{170}$ I. A. Cali, ${ }^{170}$ M. D'Alfonso, ${ }^{170}$ G. Gomez Ceballos,${ }^{170}$ M. Goncharov, ${ }^{170}$ P. Harris,${ }^{170}$ D. Hsu, ${ }^{170}$ M. Hu ${ }^{170}$ M. Klute, ${ }^{170}$ D. Kovalskyi, ${ }^{170}$ Y.-J. Lee,${ }^{170}$ P. D. Luckey, ${ }^{170}$ B. Maier, ${ }^{170}$ A. C. Marini, ${ }^{170}$ C. Mcginn, ${ }^{170}$ C. Mironov, ${ }^{170}$ S. Narayanan, ${ }^{170}$ X. Niu, ${ }^{170}$ C. Paus, ${ }^{170}$ D. Rankin,,${ }^{170}$ C. Roland, ${ }^{170}$ G. Roland, ${ }^{170}$ Z. Shi, ${ }^{170}$ G. S. F. Stephans, ${ }^{170}$ K. Sumorok, ${ }^{170}$ K. Tatar, ${ }^{170}$ D. Velicanu, ${ }^{170}$ J. Wang, ${ }^{170}$ T. W. Wang, ${ }^{170}$ B. Wyslouch, ${ }^{170}$

R. M. Chatterjee, ${ }^{171}$ A. Evans, ${ }^{171}$ S. Guts, ${ }^{171, a}$ P. Hansen, ${ }^{171}$ J. Hiltbrand, ${ }^{171}$ Sh. Jain, ${ }^{171}$ Y. Kubota, ${ }^{171}$ Z. Lesko, ${ }^{171}$ J. Mans, ${ }^{171}$ M. Revering, ${ }^{171}$ R. Rusack, ${ }^{171}$ R. Saradhy, ${ }^{171}$ N. Schroeder, ${ }^{171}$ M. A. Wadud, ${ }^{171}$ J. G. Acosta, ${ }^{172}$ S. Oliveros, ${ }^{172}$ K. Bloom, ${ }^{173}$ S. Chauhan, ${ }^{173}$ D. R. Claes, ${ }^{173}$ C. Fangmeier, ${ }^{173}$ L. Finco, ${ }^{173}$ F. Golf, ${ }^{173}$ R. Kamalieddin, ${ }^{173}$ I. Kravchenko, ${ }^{173}$ J. E. Siado, ${ }^{173}$ G. R. Snow, ${ }^{173, a}$ B. Stieger, ${ }^{173}$ W. Tabb,${ }^{173}$ G. Agarwal,,${ }^{174}$ A. Godshalk, ${ }^{174}$ S. Gozpinar, ${ }^{174}$ C. Harrington, ${ }^{174}$ I. Iashvili, ${ }^{174}$ A. Kharchilava, ${ }^{174}$ C. McLean, ${ }^{174}$ D. Nguyen, ${ }^{174}$ A. Parker, ${ }^{174}$ J. Pekkanen, ${ }^{174}$ S. Rappoccio, ${ }^{174}$ B. Roozbahani, ${ }^{174}$ G. Alverson, ${ }^{175}$ E. Barberis, ${ }^{175}$ C. Freer, ${ }^{175}$ Y. Haddad,${ }^{175}$ A. Hortiangtham, ${ }^{175}$ G. Madigan, ${ }^{175}$ B. Marzocchi, ${ }^{175}$ D. M. Morse, ${ }^{175}$ T. Orimoto, ${ }^{175}$ L. Skinnari, ${ }^{175}$ A. Tishelman-Charny, ${ }^{175}$ T. Wamorkar, ${ }^{175}$ B. Wang, ${ }^{175}$ A. Wisecarver, ${ }^{175}$ D. Wood, ${ }^{175}$ S. Bhattacharya, ${ }^{176}$ J. Bueghly, ${ }^{176}$ T. Gunter, ${ }^{176}$ K. A. Hahn, ${ }^{176}$ N. Odell, ${ }^{176}$ M. H. Schmitt, ${ }^{176}$ K. Sung, ${ }^{176}$ M. Trovato, ${ }^{176}$ M. Velasco, ${ }^{176}$ R. Bucci,${ }^{177}$ N. Dev,${ }^{177}$ R. Goldouzian, ${ }^{177}$ M. Hildreth, ${ }^{177}$ K. Hurtado Anampa,${ }^{177}$ C. Jessop, ${ }^{177}$ D. J. Karmgard, ${ }^{177}$ K. Lannon, ${ }^{177}$ W. Li, ${ }^{177}$ N. Loukas, ${ }^{177}$ N. Marinelli, ${ }^{177}$ I. Mcalister, ${ }^{177}$ F. Meng, ${ }^{177}$ C. Mueller, ${ }^{177}$ Y. Musienko, ${ }^{177, \mathrm{~mm}}$ M. Planer, ${ }^{177}$ R. Ruchti, ${ }^{177}$ P. Siddireddy, ${ }^{177}$ G. Smith, ${ }^{177}$ S. Taroni, ${ }^{177}$ M. Wayne, ${ }^{177}$ A. Wightman, ${ }^{177}$ M. Wolf,,${ }^{177}$ A. Woodard, ${ }^{177}$ J. Alimena, ${ }^{178}$ B. Bylsma, ${ }^{178}$ L. S. Durkin, ${ }^{178}$ B. Francis, ${ }^{178}$ C. Hill, ${ }^{178}$ W. Ji, ${ }^{178}$ A. Lefeld, ${ }^{178}$ T. Y. Ling, ${ }^{178}$ B. L. Winer, ${ }^{178}$ G. Dezoort, ${ }^{179}$ P. Elmer, ${ }^{179}$ J. Hardenbrook, ${ }^{179}$ N. Haubrich, ${ }^{179}$

S. Higginbotham, ${ }^{179}$ A. Kalogeropoulos, ${ }^{179}$ S. Kwan, ${ }^{179}$ D. Lange, ${ }^{179}$ M. T. Lucchini, ${ }^{179}$ J. Luo, ${ }^{179}$ D. Marlow, ${ }^{179}$ K. Mei, ${ }^{179}$ I. Ojalvo, ${ }^{179}$ J. Olsen, ${ }^{179}$ C. Palmer, ${ }^{179}$ P. Piroué, ${ }^{179}$ D. Stickland, ${ }^{179}$ C. Tully, ${ }^{179}$ Z. Wang, ${ }^{179}$ S. Malik, ${ }^{180}$ S. Norberg, ${ }^{180}$ A. Barker, ${ }^{181}$ V. E. Barnes, ${ }^{181}$ S. Das, ${ }^{181}$ L. Gutay, ${ }^{181}$ M. Jones, ${ }^{181}$ A. W. Jung, ${ }^{181}$ A. Khatiwada, ${ }^{181}$ B. Mahakud, ${ }^{181}$ D. H. Miller, ${ }^{181}$ G. Negro, ${ }^{181}$ N. Neumeister, ${ }^{181}$ C. C. Peng, ${ }^{181}$ S. Piperov,${ }^{181}$ H. Qiu, ${ }^{181}$ J. F. Schulte, ${ }^{181}$ N. Trevisani, ${ }^{181}$ F. Wang, ${ }^{181}$ R. Xiao, ${ }^{181}$ W. Xie, ${ }^{181}$ T. Cheng, ${ }^{182}$ J. Dolen, ${ }^{182}$ N. Parashar, ${ }^{182}$ U. Behrens, ${ }^{183}$ K. M. Ecklund, ${ }^{183}$ S. Freed, ${ }^{183}$ F. J. M. Geurts, ${ }^{183}$ M. Kilpatrick, ${ }^{183}$ Arun Kumar, ${ }^{183}$ W. Li, ${ }^{183}$ B. P. Padley, ${ }^{183}$ R. Redjimi, ${ }^{183}$ J. Roberts,${ }^{183}$ J. Rorie, ${ }^{183}$ W. Shi, ${ }^{183}$ A. G. Stahl Leiton, ${ }^{183}$ Z. Tu, ${ }^{183}$ A. Zhang, ${ }^{183}$ A. Bodek, ${ }^{184}$ P. de Barbaro, ${ }^{184}$ R. Demina, ${ }^{184}$ J. L. Dulemba, ${ }^{184}$ C. Fallon, ${ }^{184}$ T. Ferbel, ${ }^{184}$ M. Galanti, ${ }^{184}$ A. Garcia-Bellido, ${ }^{184}$ O. Hindrichs, ${ }^{184}$ A. Khukhunaishvili, ${ }^{184}$ E. Ranken, ${ }^{184}$ R. Taus, ${ }^{184}$ B. Chiarito, ${ }^{185}$ J. P. Chou, ${ }^{185}$ A. Gandrakota, ${ }^{185}$ Y. Gershtein,${ }^{185}$ E. Halkiadakis, ${ }^{185}$ A. Hart, ${ }^{185}$ M. Heindl, ${ }^{185}$ E. Hughes, ${ }^{185}$ S. Kaplan, ${ }^{185}$ I. Laflotte, ${ }^{185}$ A. Lath, ${ }^{185}$ R. Montalvo, ${ }^{185}$ K. Nash, ${ }^{185}$ M. Osherson, ${ }^{185}$ H. Saka, ${ }^{185}$ S. Salur, ${ }^{185}$ S. Schnetzer, ${ }^{185}$ S. Somalwar, ${ }^{185}$ R. Stone,${ }^{185}$ S. Thomas, ${ }^{185}$ H. Acharya, ${ }^{186}$ A. G. Delannoy, ${ }^{186}$ S. Spanier,${ }^{186}$ O. Bouhali, ${ }^{187, z z z}$ M. Dalchenko, ${ }^{187}$ M. De Mattia, ${ }^{187}$ A. Delgado, ${ }^{187}$ S. Dildick, ${ }^{187}$ R. Eusebi,${ }^{187}$ J. Gilmore, ${ }^{187}$ T. Huang, ${ }^{187}$

T. Kamon, ${ }^{187, \text { aaaa }}$ H. Kim, ${ }^{187}$ S. Luo, ${ }^{187}$ S. Malhotra ${ }^{187}$ D. Marley ${ }^{187}$ R. Mueller, ${ }^{187}$ D. Overton,${ }^{187}$ L. Perniè, ${ }^{187}$ D. Rathjens, ${ }^{187}$ A. Safonov, ${ }^{187}$ N. Akchurin, ${ }^{188}$ J. Damgov, ${ }^{188}$ F. De Guio, ${ }^{188}$ V. Hegde, ${ }^{188}$ S. Kunori, ${ }^{188}$ K. Lamichhane, ${ }^{188}$ S. W. Lee, ${ }^{188}$ T. Mengke, ${ }^{188}$ S. Muthumuni, ${ }^{188}$ T. Peltola, ${ }^{188}$ S. Undleeb, ${ }^{188}$ I. Volobouev, ${ }^{188}$ Z. Wang, ${ }^{188}$ A. Whitbeck, ${ }^{188}$ S. Greene, ${ }^{189}$ A. Gurrola ${ }^{189}$ R. Janjam, ${ }^{189}$ W. Johns, ${ }^{189}$ C. Maguire, ${ }^{189}$ A. Melo, ${ }^{189}$ H. Ni ${ }^{189}$ K. Padeken, ${ }^{189}$ F. Romeo, ${ }^{189}$ P. Sheldon, ${ }^{189}$ S. Tuo, ${ }^{189}$ J. Velkovska, ${ }^{189}$ M. Verweij, ${ }^{189}$ M. W. Arenton, ${ }^{190}$ P. Barria,${ }^{190}$ B. Cox,${ }^{190}$ G. Cummings,${ }^{190}$ J. Hakala, ${ }^{190}$ R. Hirosky, ${ }^{190}$ M. Joyce, ${ }^{190}$ A. Ledovskoy, ${ }^{190}$ C. Neu, ${ }^{190}$ B. Tannenwald, ${ }^{190}$ Y. Wang, ${ }^{190}$ E. Wolfe,${ }^{190}$ F. Xia, ${ }^{190}$ R. Harr, ${ }^{191}$ P. E. Karchin, ${ }^{191}$ N. Poudyal, ${ }^{191}$ J. Sturdy, ${ }^{191}$ P. Thapa,${ }^{191}$ T. Bose, ${ }^{192}$ J. Buchanan, ${ }^{192}$ C. Caillol, ${ }^{192}$ D. Carlsmith, ${ }^{192}$ S. Dasu, ${ }^{192}$ I. De Bruyn, ${ }^{192}$ L. Dodd,${ }^{192}$ C. Galloni, ${ }^{192}$ H. He ${ }^{192}$ M. Herndon, ${ }^{192}$ A. Hervé, ${ }^{192}$ U. Hussain, ${ }^{192}$ P. Klabbers, ${ }^{192}$ A. Lanaro, ${ }^{192}$ A. Loeliger, ${ }^{192}$ K. Long, ${ }^{192}$ R. Loveless, ${ }^{192}$ J. Madhusudanan Sreekala, ${ }^{192}$ D. Pinna, ${ }^{192}$ T. Ruggles, ${ }^{192}$ A. Savin, ${ }^{192}$ V. Sharma, ${ }^{192}$ W. H. Smith, ${ }^{192}$ D. Teague, ${ }^{192}$ S. Trembath-reichert, ${ }^{192}$ and N. Woods ${ }^{192}$

(CMS Collaboration)

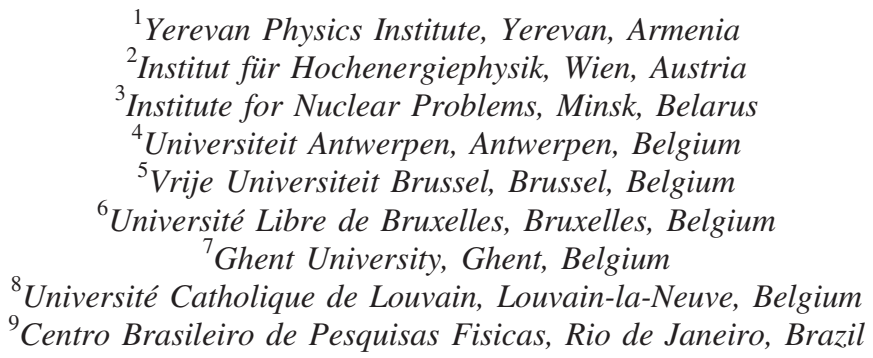


${ }^{10}$ Universidade do Estado do Rio de Janeiro, Rio de Janeiro, Brazil

${ }^{11 a}$ Universidade Estadual Paulista, São Paulo, Brazil

${ }^{11 \mathrm{~b}}$ Universidade Federal do ABC, São Paulo, Brazil

${ }^{12}$ Institute for Nuclear Research and Nuclear Energy, Bulgarian Academy of Sciences, Sofia, Bulgaria

${ }^{13}$ University of Sofia, Sofia, Bulgaria

${ }^{14}$ Beihang University, Beijing, China

${ }^{15}$ Department of Physics, Tsinghua University, Beijing, China

${ }^{16}$ Institute of High Energy Physics, Beijing, China

${ }^{17}$ State Key Laboratory of Nuclear Physics and Technology, Peking University, Beijing, China

${ }^{18}$ Zhejiang University, Hangzhou, China

${ }^{19}$ Universidad de Los Andes, Bogota, Colombia

${ }^{20}$ Universidad de Antioquia, Medellin, Colombia

${ }^{21}$ University of Split, Faculty of Electrical Engineering, Mechanical Engineering and Naval Architecture, Split, Croatia

${ }^{22}$ University of Split, Faculty of Science, Split, Croatia

${ }^{23}$ Institute Rudjer Boskovic, Zagreb, Croatia

${ }^{24}$ University of Cyprus, Nicosia, Cyprus

${ }^{25}$ Charles University, Prague, Czech Republic

${ }^{26}$ Escuela Politecnica Nacional, Quito, Ecuador

${ }^{27}$ Universidad San Francisco de Quito, Quito, Ecuador

${ }^{28}$ Academy of Scientific Research and Technology of the Arab Republic of Egypt,

Egyptian Network of High Energy Physics, Cairo, Egypt

${ }^{29}$ National Institute of Chemical Physics and Biophysics, Tallinn, Estonia

${ }^{30}$ Department of Physics, University of Helsinki, Helsinki, Finland

${ }^{31}$ Helsinki Institute of Physics, Helsinki, Finland

${ }^{32}$ Lappeenranta University of Technology, Lappeenranta, Finland

${ }^{33}$ IRFU, CEA, Université Paris-Saclay, Gif-sur-Yvette, France

${ }^{34}$ Laboratoire Leprince-Ringuet, CNRS/IN2P3, Ecole Polytechnique, Institut Polytechnique de Paris

${ }^{35}$ Université de Strasbourg, CNRS, IPHC UMR 7178, Strasbourg, France

${ }^{36}$ Centre de Calcul de l'Institut National de Physique Nucleaire et de Physique des Particules, CNRS/IN2P3, Villeurbanne, France

${ }^{37}$ Université de Lyon, Université Claude Bernard Lyon 1, CNRS-IN2P3, Institut de Physique Nucléaire de Lyon, Villeurbanne, France

${ }^{38}$ Georgian Technical University, Tbilisi, Georgia

${ }^{39}$ Tbilisi State University, Tbilisi, Georgia

${ }^{40}$ RWTH Aachen University, I. Physikalisches Institut, Aachen, Germany

${ }^{41}$ RWTH Aachen University, III. Physikalisches Institut A, Aachen, Germany

${ }^{42}$ RWTH Aachen University, III. Physikalisches Institut B, Aachen, Germany

${ }^{43}$ Deutsches Elektronen-Synchrotron, Hamburg, Germany

${ }^{44}$ University of Hamburg, Hamburg, Germany

${ }^{45}$ Karlsruher Institut fuer Technologie, Karlsruhe, Germany

${ }^{46}$ Institute of Nuclear and Particle Physics (INPP), NCSR Demokritos, Aghia Paraskevi, Greece

${ }^{47}$ National and Kapodistrian University of Athens, Athens, Greece

${ }^{48}$ National Technical University of Athens, Athens, Greece

${ }^{49}$ University of Ioánnina, Ioánnina, Greece

${ }^{50}$ MTA-ELTE Lendület CMS Particle and Nuclear Physics Group, Eötvös Loránd University, Budapest, Hungary

${ }^{51}$ Wigner Research Centre for Physics, Budapest, Hungary

${ }^{52}$ Institute of Nuclear Research ATOMKI, Debrecen, Hungary

${ }^{53}$ Institute of Physics, University of Debrecen, Debrecen, Hungary

${ }^{54}$ Eszterhazy Karoly University, Karoly Robert Campus, Gyongyos, Hungary

${ }^{55}$ Indian Institute of Science (IISc), Bangalore, India

${ }^{56}$ National Institute of Science Education and Research, HBNI, Bhubaneswar, India

${ }^{57}$ Panjab University, Chandigarh, India

${ }^{58}$ University of Delhi, Delhi, India

${ }^{59}$ Saha Institute of Nuclear Physics, HBNI, Kolkata,India

${ }^{60}$ Indian Institute of Technology Madras, Madras, India

${ }^{61}$ Bhabha Atomic Research Centre, Mumbai, India

${ }^{62}$ Tata Institute of Fundamental Research-A, Mumbai, India

${ }^{63}$ Tata Institute of Fundamental Research-B, Mumbai, India 
${ }^{64}$ Indian Institute of Science Education and Research (IISER), Pune, India

${ }^{65}$ Institute for Research in Fundamental Sciences (IPM), Tehran, Iran

${ }^{66}$ University College Dublin, Dublin, Ireland

${ }^{67 a}$ INFN Sezione di Bari

${ }^{67 \mathrm{~b}}$ Università di Bari

${ }^{67 \mathrm{c}}$ Politecnico di Bari

${ }^{68 \mathrm{a}}$ INFN Sezione di Bologna, Bologna, Italy

${ }^{68 \mathrm{~b}}$ Università di Bologna, Bologna, Italy

${ }^{69 a}$ INFN Sezione di Catania, Catania, Italy

${ }^{69 \mathrm{~b}}$ Università di Catania, Catania, Italy

${ }^{70 \mathrm{a}}$ INFN Sezione di Firenze, Firenze, Italy

${ }^{70 \mathrm{~b}}$ Università di Firenze, Firenze, Italy

${ }^{71}$ INFN Laboratori Nazionali di Frascati, Frascati, Italy

${ }^{72 a}$ INFN Sezione di Genova, Genova, Italy

${ }^{72 \mathrm{~b}}$ Università di Genova, Genova, Italy

${ }^{73 a}$ INFN Sezione di Milano-Bicocca, Milano, Italy

${ }^{73 b}$ Università di Milano-Bicocca, Milano, Italy

${ }^{74 a}$ INFN Sezione di Napoli, Roma, Italy

${ }^{74 b}$ Università di Napoli 'Federico II', Roma, Italy

${ }^{74 c}$ Università della Basilicata, Roma, Italy

${ }^{74 \mathrm{~d}}$ Università G. Marconi, Roma, Italy

${ }^{75 a}$ INFN Sezione di Padova, Trento, Italy

${ }^{75 b}$ Università di Padova, Trento, Italy

${ }^{75 \mathrm{c}}$ Università di Trento, Trento, Italy

${ }^{76 a}$ INFN Sezione di Pavia, Trento, Italy

${ }^{76 b}$ Università di Pavia, Trento, Italy

${ }^{77 a}$ INFN Sezione di Perugia, Perugia, Italy

${ }^{77 \mathrm{~b}}$ Università di Perugia, Perugia, Italy

${ }^{78 a}$ INFN Sezione di Pisa, Pisa, Italy

${ }^{78 b}$ Università di Pisa, Pisa, Italy

${ }^{78 \mathrm{c}}$ Scuola Normale Superiore di Pisa, Pisa, Italy

${ }^{79 a}$ INFN Sezione di Roma, Rome, Italy

${ }^{79 b}$ Sapienza Università di Roma, Rome, Italy

${ }^{80 \mathrm{a}}$ INFN Sezione di Torino, Novara, Italy

${ }^{80 \mathrm{~b}}$ Università di Torino, Novara, Italy

${ }^{80 \mathrm{c}}$ Università del Piemonte Orientale, Novara, Italy

${ }^{81 a}$ INFN Sezione di Trieste, Trieste, Italy

${ }^{81 \mathrm{~b}}$ Università di Trieste, Trieste, Italy

${ }^{82}$ Kyungpook National University, Daegu, Korea

${ }^{83}$ Chonnam National University, Institute for Universe and Elementary Particles, Kwangju, Korea

${ }^{84}$ Hanyang University, Seoul, Korea

${ }^{85}$ Korea University, Seoul, Korea

${ }^{86}$ Kyung Hee University, Department of Physics

${ }^{87}$ Sejong University, Seoul, Korea

${ }^{88}$ Seoul National University, Seoul, Korea

${ }^{89}$ University of Seoul, Seoul, Korea

${ }^{90}$ Sungkyunkwan University, Suwon, Korea

${ }^{91}$ Riga Technical University, Riga, Latvia

${ }^{92}$ Vilnius University, Vilnius, Lithuania

${ }^{93}$ National Centre for Particle Physics, Universiti Malaya, Kuala Lumpur, Malaysia

${ }^{94}$ Universidad de Sonora (UNISON), Hermosillo, Mexico

${ }^{95}$ Centro de Investigacion y de Estudios Avanzados del IPN, Mexico City, Mexico

${ }^{96}$ Universidad Iberoamericana, Mexico City, Mexico

${ }^{97}$ Benemerita Universidad Autonoma de Puebla, Puebla, Mexico

${ }^{98}$ Universidad Autónoma de San Luis Potosí, San Luis Potosí, Mexico

${ }^{99}$ University of Montenegro, Podgorica, Montenegro

${ }^{100}$ University of Auckland, Auckland, New Zealand

${ }^{101}$ University of Canterbury, Christchurch, New Zealand

${ }^{102}$ National Centre for Physics, Quaid-I-Azam University, Islamabad, Pakistan 


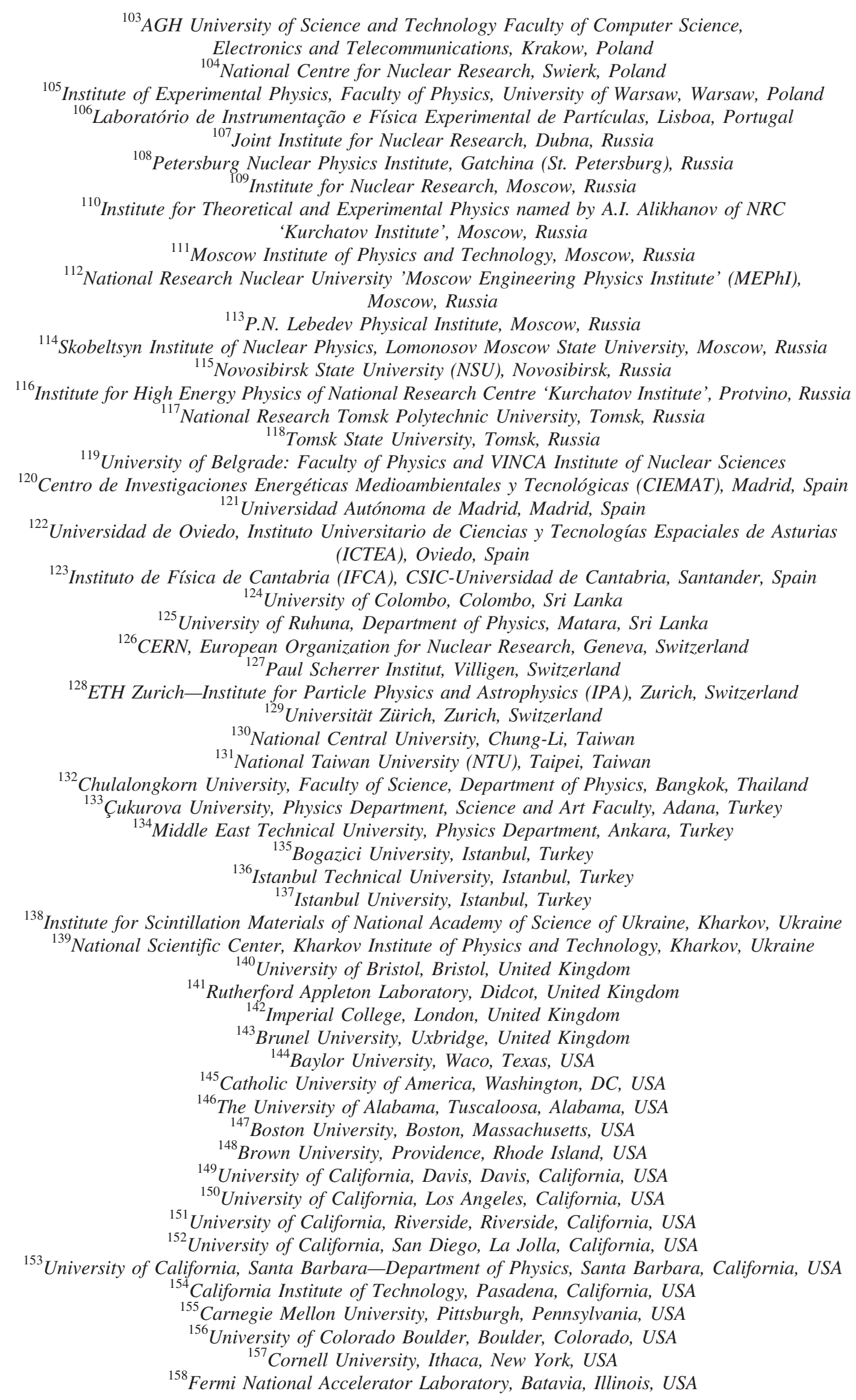




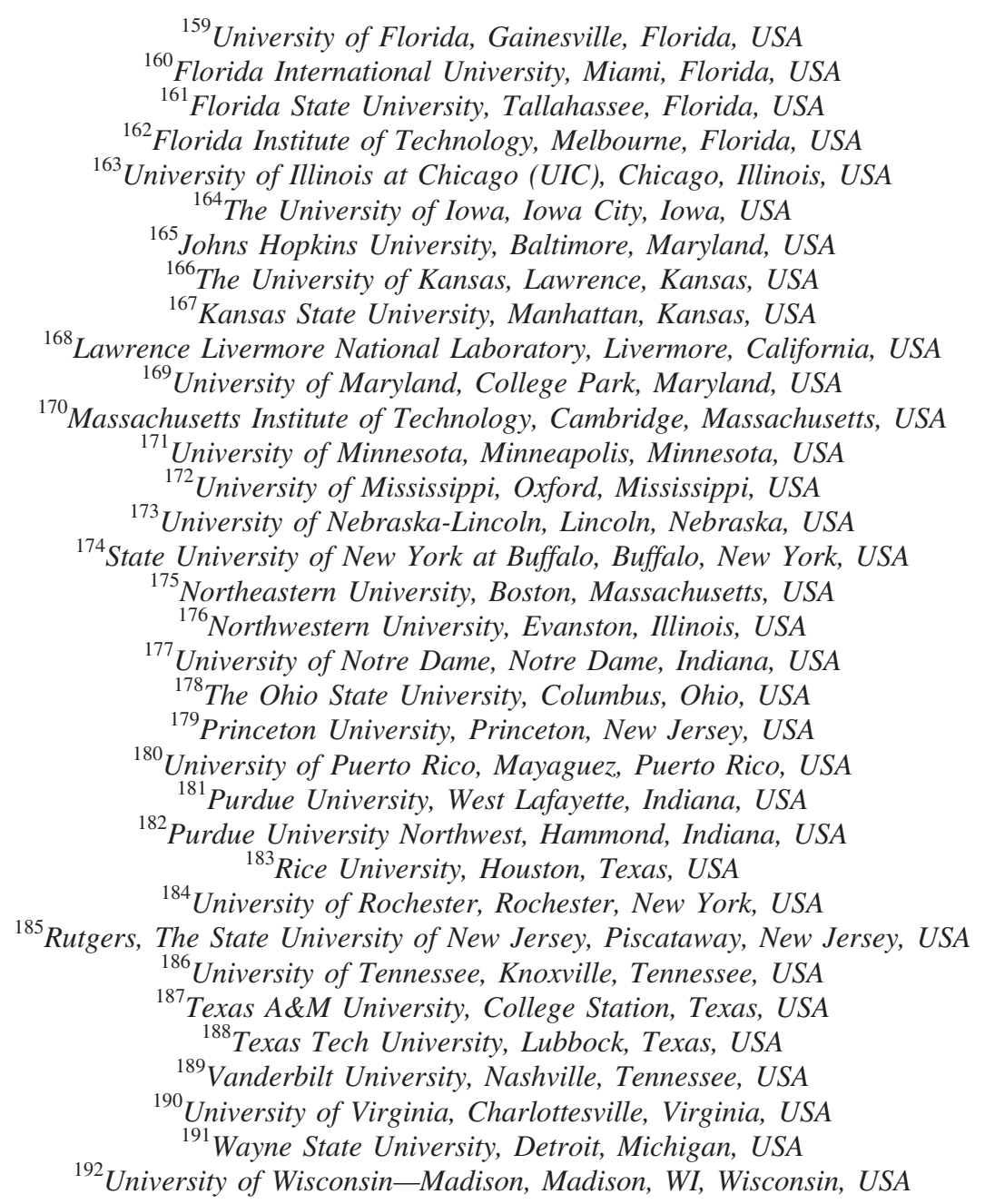

${ }^{\mathrm{a}}$ Deceased.

${ }^{\mathrm{b}}$ Also at Vienna University of Technology, Vienna, Austria.

${ }^{\mathrm{c}}$ Also at IRFU, CEA, Université Paris-Saclay, Gif-sur-Yvette, France.

${ }^{\mathrm{d}}$ Also at Universidade Estadual de Campinas, Campinas, Brazil.

${ }^{\mathrm{e}}$ Also at Federal University of Rio Grande do Sul, Porto Alegre, Brazil.

${ }^{\mathrm{f}}$ Also at UFMS.

${ }^{\mathrm{g}}$ Also at Universidade Federal de Pelotas, Pelotas, Brazil.

${ }^{\mathrm{h}}$ Also at Université Libre de Bruxelles, Bruxelles, Belgium.

${ }^{\mathrm{i}}$ Also at University of Chinese Academy of Sciences.

${ }^{\mathrm{j}}$ Also at Institute for Theoretical and Experimental Physics named by A.I. Alikhanov of NRC 'Kurchatov Institute', Moscow, Russia.

${ }^{\mathrm{k}}$ Also at Joint Institute for Nuclear Research, Dubna, Russia.

${ }^{1}$ Also at British University in Egypt, Cairo, Egypt.

${ }^{\mathrm{m}}$ Also at Ain Shams University, Cairo, Egypt.

${ }^{\mathrm{n}}$ Also at Purdue University, West Lafayette, Indiana, USA.

${ }^{\circ}$ Also at Université de Haute Alsace, Mulhouse, France.

${ }^{\mathrm{p}}$ Also at Tbilisi State University, Tbilisi, Georgia.

${ }^{\mathrm{q}}$ Also at Erzincan Binali Yildirim University, Erzincan, Turkey.

${ }^{r}$ Also at CERN, European Organization for Nuclear Research, Geneva, Switzerland.

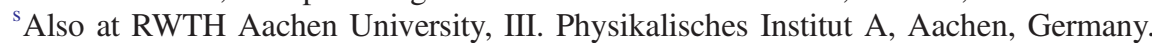

${ }^{\mathrm{t}}$ Also at University of Hamburg, Hamburg, Germany.

${ }^{\mathrm{u}}$ Also at Brandenburg University of Technology, Cottbus, Germany.

${ }^{v}$ Also at Institute of Physics, University of Debrecen, Debrecen, Hungary.

${ }^{\mathrm{w}}$ Also at Institute of Nuclear Research ATOMKI, Debrecen, Hungary.

${ }^{x}$ Also at MTA-ELTE Lendület CMS Particle and Nuclear Physics Group, Eötvös Loránd University, Budapest, Hungary.

${ }^{\mathrm{y}}$ Also at IIT Bhubaneswar, Bhubaneswar, India. 
${ }^{\mathrm{z}}$ Also at Institute of Physics, Bhubaneswar, India.

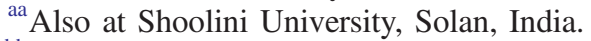

${ }^{\mathrm{bb}}$ Also at University of Hyderabad, Hyderabad, India.

${ }^{\mathrm{cc}}$ Also at University of Visva-Bharati, Santiniketan, India.

${ }^{\mathrm{dd}}$ Also at Isfahan University of Technology.

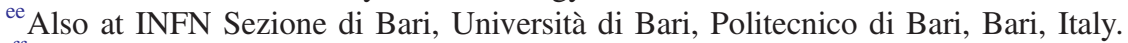

${ }^{\mathrm{ff}}$ Also at Italian National Agency for New Technologies, Energy and Sustainable Economic Development.

${ }^{g g}$ Also at Centro Siciliano di Fisica Nucleare e di Struttura Della Materia.

${ }^{\text {hh }}$ Also at Scuola Normale e Sezione dell'INFN, Pisa, Italy.

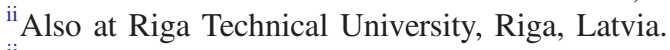

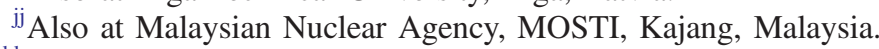

${ }^{\mathrm{kk}}$ Also at Consejo Nacional de Ciencia y Tecnología, Mexico City, Mexico.

${ }^{11}$ Also at Warsaw University of Technology, Institute of Electronic Systems, Warsaw, Poland.

${ }^{\mathrm{mm}}$ Also at Institute for Nuclear Research, Moscow, Russia.

${ }^{\mathrm{nn}}$ Also at National Research Nuclear University 'Moscow Engineering Physics Institute' (MEPhI), Moscow, Russia.

${ }^{\text {oo }}$ Also at St. Petersburg State Polytechnical University, St. Petersburg, Russia.

${ }^{\mathrm{pp}}$ Also at University of Florida, Gainesville, Florida, USA.

${ }^{\mathrm{qq}}$ Also at Imperial College, London, United Kingdom.

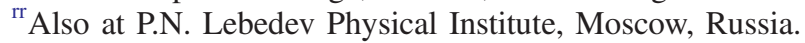

${ }^{\text {ss }}$ Also at California Institute of Technology, Pasadena, California, USA.

${ }^{t t}$ Also at Budker Institute of Nuclear Physics, Novosibirsk, Russia.

${ }^{\mathrm{uu}}$ Also at Faculty of Physics, University of Belgrade, Belgrade, Serbia.

${ }^{\mathrm{vv}}$ Also at Università degli Studi di Siena, Siena, Italy.

${ }^{\text {ww }}$ Also at INFN Sezione di Pavia, Università di Pavia, Pavia, Italy.

${ }^{\mathrm{xx}}$ Also at National and Kapodistrian University of Athens, Athens, Greece.

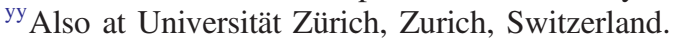

${ }^{z z}$ Also at Stefan Meyer Institute for Subatomic Physics, Vienna, Austria.

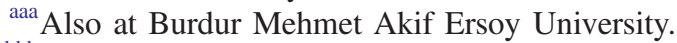

${ }^{b b b}$ Also at Adiyaman University, Adiyaman, Turkey.

${ }^{\mathrm{ccc}}$ Also at Şırnak University.

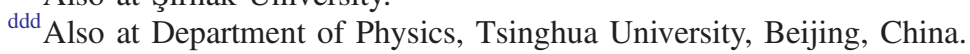

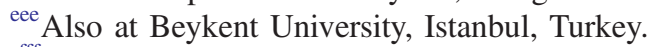

${ }^{\mathrm{fff}}$ Also at Istanbul Aydin University, Application and Research Center for Advanced Studies (App. \& Res. Cent. for Advanced Studies).

${ }^{\text {ggg }}$ Also at Mersin University, Mersin, Turkey.

${ }^{\text {hhh }}$ Also at Piri Reis University, Istanbul, Turkey.

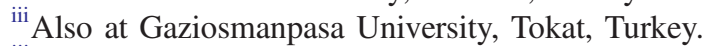

${ }_{\mathrm{jjj}}$ Also at Ozyegin University, Istanbul, Turkey.

${ }^{k k k}$ Also at Izmir Institute of Technology, Izmir, Turkey.

${ }^{111}$ Also at Marmara University, Istanbul, Turkey.

${ }^{\mathrm{mmm}}$ Also at Kafkas University, Kars, Turkey.

${ }^{n n n}$ Also at Istanbul Bilgi University, Istanbul, Turkey.

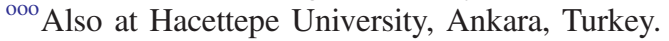

${ }^{\mathrm{ppp}}$ Also at Vrije Universiteit Brussel, Brussel, Belgium.

${ }^{\mathrm{qqq}}$ Also at School of Physics and Astronomy, University of Southampton, Southampton, United Kingdom.

${ }^{\mathrm{rrr}}$ Also at IPPP Durham University.

${ }^{\text {sss }}$ Also at Monash University, Faculty of Science, Clayton, Australia.

${ }^{\text {ttt }}$ Also at Bethel University, St. Paul, Minneapolis, USA.

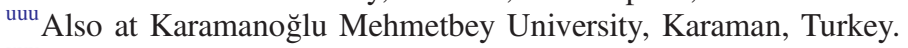

${ }^{\mathrm{vvv}}$ Also at Bingol University, Bingol, Turkey

${ }^{w w w}$ Also at Georgian Technical University, Tbilisi, Georgia.

${ }^{\mathrm{xxx}}$ Also at Sinop University, Sinop, Turkey.

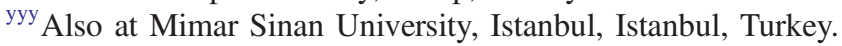

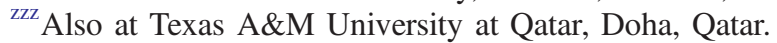

${ }^{\text {aaa }}$ Also at Kyungpook National University, Daegu, Korea. 